\title{
LICHENS de la RÉGION d'OTTAWA
}

IRWIN M. BRODO

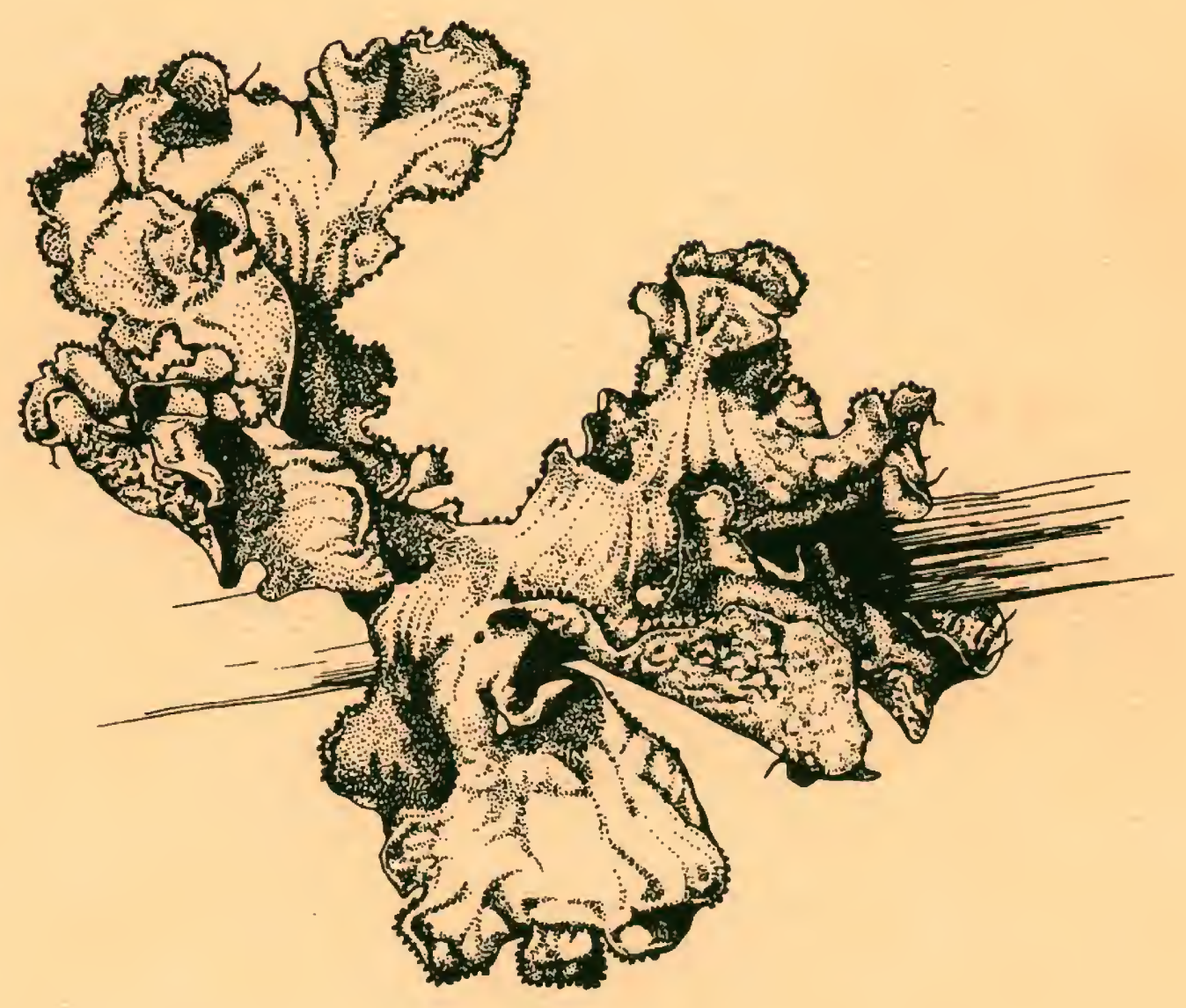


La collection SyLLoGeus, publiêe par le Musée national des Sciences naturelles, Musées nationaux du Canada, a pour but de diffuser rapidement le résultat des travaux dans les domaines scientifique et educatif qui sont sous la direction du Musee national des sciences naturelles. Pour assurer la prompte distribution de cette publication, on a abrégé les étapes de la rédaction.

Les articles sont publiés en français, en anglais ou dans les deux langues, et ils paraissent irrégulièrement. On peut obtenir par commande postale la liste des titres de tous les articles publiés depuis le début de la collection (1972) et des copies individuelles de ce numéro, au Musée national des sciences naturelles, Ottawa, Canada. KlA 0 M 8 .

SYLLOGEUS is a publication of the National Museum of Natural Sciences, National Museums of Canada, designed to permit the rapid dissemination of information pertaining to those disciplines and educational functions for which the national Museum of Natural Sciences is responsible. In the interests of making information available quickly, normal publishing procedures have been abbreviated.

Articles are published in English, in French, or in both languages, and the issues appear at irregular intervals. A complete list of the titles issued since the beginning of the series (1972) and individual copies of this number are available by mail from the National Museum of Natural Sciences, Ottawa, Canada. KIA $0 M 8$.

L'édition anglaise de cette publication existe sous le titre Lichens of the Ottawa Region.

The English edition of this publication is available under the title Lichens of the ottawa Region.

Syllogeus series No. 29 (French edition)

(c) National Museums of Canada 1981

Printed in Canada

ISSN $0704-576 \mathrm{X}$
Série Syllogeus no 29 (L'édition

française)

(c) Musées nationaux du Canada 1981

Imprimé au Canada

ISSN $0704-576 \mathrm{X}$ 
LICHENS DE LA RÉGION D'OTTAWA

\author{
par Irwin M. Brodo \\ Division de la Botanique \\ Musée national des Sciences naturelles \\ ottawa, ontario KlA OM8 \\ traduit de l'anglais
par Sylvie Lemieux
}

Musée national des Sciences naturelles Musées nationaux du Canada

National Museum of Natural Sciences National Museums of Canada Ottawa 1981 


\section{RÉSUME}

La ville d'ottawa, situe dans l'est de l'Ontario approximativement à mi-chemin entre Québec et Toronto, se trouve dans la région des forêts décidues. La flore lichénique, dans un rayon de 50 kilomètres autour d'Ottawa, comprend environ 370 espèces de lichens, et elle est véritablement représentative de cette région peuplée dans son entier.

Cet ouvrage présente d'abord une discussion générale sur les lichens en tant qu'organismes et fournit des instructions concernant leur collection, leur préservation et leur étude. Ensuite apparaîssent des clefs diagnostiques des genres des lichens crustacés basêes sur des spécimens fertiles ou stériles. Alors, il y a une série des clefs pour identifier les espèces individuelles; ces clefs sont classés selon les genres. Les clefs des lichens foliacés et des lichens fruticuleux terminent cette section. Un glossaire de termes spéciaux, employés dans les clefs, constitue une annexe, ainsi qu'un répertoire des espèces comprises dans l'ouvrage. Les clefs et le glossaire comprennent 75 dessins au trait qui représentent plusieurs lichens et quelques-unes de leurs caractéristiques microscopiques.

\section{ABSTRACT}

The city of ottawa is situated in eastern Ontario approximately half way between Quebec City and Toronto in the deciduous forest region. The 1 ichen flora within a 50-kilometre radius of ottawa consists of about 370 species of lichens, and is fairly representative of this entire populated corridor.

This work opens with a short general discussion of lichens as organisms, including pointers on their collection, preservation, and study. Diagnostic keys are then presented to the genera of crustose lichens based on fertile or on sterile material. Individual species are then keyed out within their genera. keys to foliose lichens and fruticose lichens (without respect to particular genera) complete the section. A glossary of special terms used in the keys is presented as an appendix, together with an index of the species covered. The keys and glossary are illustrated with 75 black-and-white drawings depicting many of the lichens and some of their microscopic features. 
TABLE DES MATIERES

Prêface 5

Introduction 6

Quelques principes gểnēraux 6

Un mot sur quelques travaux antérieurs au présent
document et sur celui-ci

L'utilisation des clefs 8

La récolte et l'étude des lichens 10

$\begin{array}{lr}\text { Remerciements } & 14\end{array}$

Les genres crustacés fertiles 16

Les espèces crustacées et squamuleuses stériles 30

Genres des lichens crustacẻs (clefs aux espèces)

$\begin{array}{lr}\text { Acarospora } & 37\end{array}$

Arthonia 237

Arthopyrenia 8.2 . 38

Arthothelium $\quad 39$

Aspicilia $\quad 39$

$\begin{array}{lr}\text { Bacidia } & 40\end{array}$

Buellia $r 44$

Caliciales $r$

Caloplaca $r$

Candelarielza $r$

Dimerezla $r$

$\begin{array}{lr}\text { Huilia } & 50\end{array}$

Lecania $r$

Lecanora 51

Lecidea s.z. $r$

Micarea $r$

ochrolechia $r$

$\begin{array}{lr}\text { Pertusaria } & 64\end{array}$

$\begin{array}{lr}\text { Plagiocarpa } & 67\end{array}$

$\begin{array}{lr}\text { Psora \& Hypocenomyce } & 67\end{array}$

$\begin{array}{lr}\text { Pyrenuza } & 68\end{array}$

Rhizocarpon $r$

Rinodina $r$

$\begin{array}{ll}\text { Sarcogyne } & 72\end{array}$

Staurothele $\quad 73$

$\begin{array}{lr}\text { Verucaria } & 73\end{array}$

Les lichens foliacés (synopsis) 76

Les lichens foliacés (clef gênérale) 78

$\begin{array}{ll}\text { Les lichens fruticuleux (synopsis) } & 108\end{array}$

Les lichens fruticuleux (clef générale) 109

Références $\quad 124$

$\begin{array}{lr}\text { Glossaire } & 125\end{array}$

$\begin{array}{lr}\text { Liste et index des lichens } & 134\end{array}$ 


\section{Figures}

1-4 1. Acarospora fuscata, 2. Aspicizia cinerea,

3. Candelariella efflorescens, 4. C. xanthostigma

5-10 5. Calicium trabinellum, 6. Mycocalicium parietinum,

7. Cyphelium tigizlare, 8. Conotrema urceolatum,

9. Graphis seripta, 10. Arthonia radiata

11-13 11. Bacidia schweinitzii, 12. Scoliciosporum

chlorococcum, 13. Bacidia sabuletorum

14-15 14. Caloplaca feracissima, 15. C. holocarpa

16-18 16. Lecanora rugoselza, 17. L. pulicaris,

18. L. glabrata

19-21 19. Lecidea erratica, 20. Huilia macrocarpa,

21. Hypocenomyce scalaris

22-27' 22. Dimelaena oreina, 23. Lecanora glabrata,

24. Pertusaria macounii, 25. Sarcogyne simplex,

26. Staurothele fissa, 27. Verrucaria calciseda

28-30 28. Rinodina bischoffii, 29. R. dakotensis,

30. R. halei

31-32 31. Xanthoria fallax, 32. Parmelia cumberlandia

33-37 33. Colzema, coupe verticale du thalle, 34. C. subflaccidum, 35. Leptogium, coupe

verticale du thalle, 36. Leptogium cyanescens, 37. L. Zichenoides

38-41 38. Peltigera rufescens, 39. Dermatocarpon weberi, 40. Lasaliza papulosa, 41. Solorina saceata

42-43 42. Hypogymnia physodes, 43. Lobaria pulmonaria

44-48 44. Phaeophyscia rubropulchra, 45. Physcia

millegrana, 46. Heterodermia speciosa, 47. Parmelia sorediosa, 48 . P. disjuncta

49-50 49. Parmelia sulcata, 50. Cetraria arenaria

51-52 51. Cetraria halei, 52. Physcia aipolia

53-58 53. Cladina stellaris, 54. C. mitis, 55. Cladonia

phylzophora, 56. C. cervicornis subsp. verticillata, 57. C. coniocraea, 58. C. chlorophaea s.l.

59-64 59. Stereocaulon saxatile, 60. Baeomyces rufus,

61. Usnea hirta, 62. Bryoria furcellata,

63. Ramalina intermedia, 64. Evernia mesomorpha

65-74 Illustrations pour la glossaire 
PRÉFACE

En 1967, deux articles que j'avais rédigés sur la détermination des lichens ont paru dans la revue du Ottawa Field-Naturalists' Club, Trail \& Landscape (Brodo, 1967a, b). Le troisième et dernier article de la série a été publié dans le même périodique en 1972 (Brodo, 1972). C'est en pensant au naturaliste amateur mais sérieux que j'avais écrit ces lignes; par conséquent, j'avais employé le moins de termes scientifiques possible et j'avais réduit les techniques au strict minimum. Pour permettre au lecteur d'identifier les lichens de la région, j'avais présenté une "clef" d'identification des espèces communes.

Depuis une dizaine d'années, on réclame ce genre de clef à cor et à cri; seulement la demande ne provient pas des botanistes amateurs pour qui ces articles avaient été composés. Le besoin semble en général se faire sentir chez les étudiants des niveaux secondaire et collégial. Ces jeunes gens semblent prêts à faire davantage usage du microscope et des réactifs que les amateurs. Qui plus est, ils désirent que les clefs renferment toutes les espèces connues dans la région au lieu de s'en tenir uniquement aux plus fréquemment rencontrées.

En raison de la demande et du besoin de clefs plus complètes, ces trois articles et clefs étaient combinés et complètement revisés. Le présent numéro de syzrogeus (disponible également en anglais) demeure, dans un sens, une version provisoire. En effet, ce n'est pas avant d'avoir poussé plus à fond l'exploration de la rêgion outaouaise que nous serons relativement certains que la flore au complet a été échantillonnée. Nous apprécierons grandement les additions et les corrections dont voudront bien nous faire part les botanistes amateurs et professionnels. 


\section{INTRODUCTION}

L'un des domaines de l'histoire naturelle du sud de l'ontario et du Québec à avoir été relativement négligé est celui de la végétation lichénique. Ces plantes intéressantes, qui s'étalent en plaques colorêes sur les rochers et sur les arbres poussant le long des chemins, méritent d'occuper une place de choix dans le calepin du naturaliste.

\section{Quelques principes généraux}

Aux yeux de l'observateur occasionnel, les lichens représentent souvent un groupe dont font partie les mousses, parfois les algues et, plus rarement, les champignons. Les gens que je rencontre sont souvent étonnés par le large éventail des formes que les lichens peuvent emprunter. Est-il possible qu'elles soient toutes des lichens? Comment cette tache noire sur ce rocher peut-elle être une proche parente de cette "mousse à caribous" buissonneuse et abondamment ramifiée? Nous esquisserons la réponse à cette question en essayant tout d'abord de répondre à une autre: qu'est-ce que c'est au juste qu'un lichen?

Le point essentiel réside dans le fait que tout lichen, contrairement aux autres vêgétaux, est en réalité constitué de deux plantes. L'appareil végétatif de chaque lichen, encore appelé "thalle", représente, au point de vue biologique, un complexe composé d'un champignon entretenant des rapports étroits avec une algue. Ce type de relation est nettement supérieur à un simple contact entre les constituants ou même à leur proximité relative; il en résulte plutôt la formation d'une toute nouvelle entité autonome qui se procure les éléments nécessaires à sa survie et fabrique sa nourriture, dont les constituants procèdent entre eux à un échange de vitamines et d'autres substances vitales, et qui souvent se reproduit comme s'il s'agissait d'une seule et même plante. De par sa forme et sa structure, le lichen diffère du champignon et de l'algue libres, c'est-à-dire "non-lichenisés". On n'a pas encore trouvé avec certitude de champignons lichéniques croissant sous la forme non lichénisée dans la nature. Par contre, bon nombre d'espèces d'algues lichéniques se retrouvent à l'état libre.

Dans la région outaouaise, l'observateur repère toutes sortes de lichens dans une grande diversité d'habitats. Ils sont lobés ou revêtent la forme de feuilles (lichens foliacés); ils adoptent l'apparence de tiges, de buissons ou de filaments (lichens fruticuleux) ou encore celle de croûtes (1ichens crustacés). La plupart des espèces s'installent à même le sol, sur les pierres, les cailloux, l'écorce ou le bois; cependant un petit nombre d'entre elles se limitent aux rochers immergés dans les cours d'eau, vivent 
sur les mousses et les écricacées en décomposition ou encore se retrouvent le long de l'océan, sur les plages de galets, sur les rochers battus par la vague ou même sur les coquilles d'anatife. Il est probable que le botaniste trouvera peu d'espèces à proximité du centre des villes ou des complexes industriels, car, cela est bien connu, les lichens sont sensibles à la pollution et à la dessication de l'atmosphère.

Un mot sur quelques travaux antërieurs au présent document et sur celui-ci

L'ouvrage sur les lichens de l'Outaouais, rédigé par John Macoun, il y a de cela 80 ans, est un classique dans le domaine (Macoun, 1898a, b, c; 1902). Premier botaniste au service du gouvernement fédéral canadien, Macoun était un excellent collectionneur. Bien que ses publications ne consistent en rien d'autre qu'en une liste de noms et qu'elles soient largement dépassées aujourd'hui, ses spécimens sont toujours conservës à l'Herbier National, têmoins importants de la flore passée et actuelle de la rêgion.

Au fur et à mesure que grandit l'intérêt pour les lichens, on assiste à la parution d'un grand nombre d'articles utiles. Certains d'entre eux, qui sont publiês dans des revues de vulgarisation canadiennes, prêsentent d'excellentes initiations à la lichénologie (Bennett, 1979; McMillan, 1979). Le naturaliste que l'identification des lichens intéresse doit faire face à un problème de taille. Il est certain que le meilleur livre traitant de notre région est la seconde édition de Mason Hale, "How to know the lichens" (Hale, 1979). Toutefois, l'ouvrage couvre un très vaste territoire (tous les États-Unis et la majeure partie du Canada); c'est pourquoi il contient beaucoup plus d'espèces qu'il n'en existe dans le sud du Canada. Ce nombre impressionnant risque de semer la confusion dans l'esprit de l'amateur. Une autre faiblesse du livre réside dans le fait qu'il traite uniquement des "macrolichens", c'est-à-dire, des espèces foliacées et fruticuleuses. En ce qui a trait à la détermination des lichens crustacés, le naturaliste doit s'en tirer tout seul.

Voilà pourquoi les botanistes ont besoin d'un guide permettant l'identification des lichens du sud du Canada. Si j'ai choisi la région outaouaise comme centre de mon étude, c'est en raison de ma bonne connaissance des lieux ainsi que du fait qu'elle est au milieu du territoire particulièrement peuplé, situé entre Québec et Toronto. Après quelques essais avec les clefs, nous avons conclu qu'elles s'appliquaient à la plus grande partie de la région forestière des Grands Lacs et du Saint-Laurent, dans le sud-est du Canada. Elles seront beaucoup moins utiles dans les Maritimes de même qu'à l'ouest du Lac Supérieur.

Les clefs qui vont suivre couvrent les quelque 370 espèces qui se 
trouvent dans un rayon de $50 \mathrm{~km}$ du centre d'ottawa. La majeure partie de la région est constituée de forêts décidues typiques, allant d'associations ouvertes de bouleau et de peuplier aux érablières sucrières à hêtre parvenues à maturité, en passant par des stations dominées par le chêne ou l'orme. Il y a également quelques groupements de conifères dans certains coins du parc de la Gatineau, du côté québécois de la rivière des Outaouais.

\section{L'utilisation des clefs}

En"raison du nombre important d'espèces traitées, les clefs ont été đivisées en sections. La première est consacrée aux espèces crustacées et inclut toute espèce vraiment crustacée (formant une croûte à la surface du substrat ou à l'intérieur de celui-ci, et si intimement fixée au support que le lichen ne peut en être détaché) de même que les taxons "formés de squames", dits squamuzeux.

Il faut noter que, chez certaines espèces "crustacées", le thalle ou l'appareil végétatif est si manifestement lobé qu'elles donnent l'impression d'être foliacêes. Quelques-unes de ces espèces intermédiaires sont décrites à la fois dans la clef des crustacés et celle des foliacés; seulement, dans le cas oủ la première clef que l'on consulte se révèle insatisfaisante, on devra alors se tourner vers la seconde.

C'est par les genres des crustacés fertiles (c.-à-d. ceux possédant des fructifications) que nous amorçons la section consacrée aux espèces crustacées. Pour les genres qui ne sont représentês que par une espèce dans la région d'ottawa, leur description se termine par une "diagnose" ou un bref signalement de l'espèce au dernier choix. (Toutes les clefs sont en réalité des diagnoses, étant donné qu'elles offrent une description abrégée du taxon dans le dernier doublet.) La clef des espèces crustacées stériles (dépourvues de fructifications) fait suite à la clef des genres fertiles. On trouve enfin la clef des espèces crustacées de chaque genre, les genres étant présentés par ordre alphabétique.

La section qui suit est consacrée aux lichens foliacés. Ceux-ci offrent l'aspect de feuilles, sont plus ou moins plats, pourvus de faces supérieure et inférieure distinctes, et généralement retenus au substratum en une multitude de points, le plus souvent, par de minuscules poils semblables à des racines et appelés rhizines. Parfois les lichens foliacês sont très fortement appliqués contre leur support et sont presque crustacés (p. ex. Physciopsis, doublet 89); par ailleurs quelques types morphologiques possèdent des lobes ascendants, presque dressés et ressemblent beaucoup à des Iichens fruticuleux ( $p$. ex. Cetraria arenaria, doublet 100).

Les lichens foliacés sont déterminés ensemble, c'est-à-dire sans tenir 
compte du genre, et cela en raison du fait qu'un grand nombre des genres identifiés à l'heure actuelle sont basés sur des caractères microscopiques ou particulièrement infimes. La longue clef gênérale est précédée d'une version abrégée ou synopsis. La majorité des usagers, surtout ceux pour qui les lichens ne sont pas des plantes familières, se rendront compte de la très grande utilité du synopsis, mais cela après qu'ils auront quelque peu pris connaissance de la clef générale.

La dernière section renferme les clefs des lichens fruticuleux; ceux-ci sont dressés ou pendants et sont retenus au substratum en quelques points, tout au plus. En règle générale, les lichens fruticuleux empruntent la forme de coussins, de paillassons ou de touffes; au sein de cette catégorie figurent les fameuses cladonies, les lichens à caribous, la "barbe à papa" et bien d'autres formes semblables. On trouve de surcroît les Baeomyces, qui se composent d'un "thalle primaire" crustacé d'où s'élèvent des tiges fruticuleuses ou "podétions". Ces espèces ont été rangées avec les lichens fruticuleux quand elles sont fertiles, et avec les lichens crustacés lorsqu'elles sont stériles. Les espèces de Cladonia ont toutes un thalle primaire qui varie de squamuleux à presque foliacé; néanmoins elles ont été mises dans la clef des lichens fruticuleux.

L'utilisation des clefs requiert donc d'abord la détermination du type morphologique du lichen étudié, puis le choix de la bonne clef parmi celles des genres de lichens crustacés fertiles ( $p$. 16), des espèces crustacées stériles (p. 30), des espèces foliacées (synopsis, p. 76; clef générale, p. 78) et des espèces fruticuleuses (synopsis, p. 108; clef générale, p. 109).

Il est évident que le gros de la terminologie est inconnu au naturaliste qui n'a jamais touché à des lichens auparavant. C'est pourquoi un glossaire illustré à été annexé à la page 125 .

Utiliser la couleur peut semer la confusion; par contre, se passer de ce critère est absurde. En fait, pour se servir de ce caractère en toute confiance, le naturaliste doit avoir de l'expérience. Il est certain que l'emploi des termes "jaunâtre" et "vert jaunâtre" est tout ce qu'il y a de plus embêtant; ils s'appliquent ordinairement aux lichens contenant un pigment jaune pâle, l'acide usnique. En règle générale, si un lichen tire un tant soit peu sur le jaune terne, même s'il serait d'emblée qualifié simplement de "vert", il est préférable de le classer au rang des thalles "jaunâtres". Une indication sur l'écologie et sur l'abondance relative est donnée à chaque espèce. J'admets volontiers que l'abondance est une notion très subjective; afin que l'on puisse comparer les indications, je n'ai utilisé que les mots "commun", "fréquent", "peu fréquent" et "rare". Par "commun", j'entends que le lichen a été trouvé à bon nombre d'endroits dans la région. 
"Fréquent" indique que j'ai récolté un certains nombres de spécimens, mais que la distribution de l'espèce a tendance à être sporadique. Par "peu fréquent", je veux dire que je n'ai que quelques spécimens de l'espèce et que l'on ne la rencontre pas souvent. "Rare" signifie que le taxon n'a été repéré qu'à une ou deux reprises dans la région. Cependant, je dois signaler que les indications sur l'abondance ne s'applique qu'à la région de l'outaouais; une espèce "rare" ici peut très bien être "commune" plus à l'est ou à l'ouest.

\section{La récolte et l'étude des lichens}

Les lichens foliacés et fruticuleux

La récolte des "macrolichens" ne pose habituellement aucune difficulté. Dans la presque totalité des cas, le botaniste peut détacher le thalle avec ou sans le substrat, à l'aide d'un bon couteau de chasse. Un sécateur s'avère pratique, mais non indispensable, pour les espèces installées sur les rameaux. L'auteur de la récolte doit glisser ses spécimens dans des sacs de papier (jamais de plastique) en ayant soin d'y inscrire à l'extérieur les conditions écologiques.

Bon nombre de macrolichens sont assez épais et fragiles; ils doivent donc subir quelques petits préparatifs pour en faire des échantillons valables. Les lichens foliacés et fruticuleux volumineux doivent être humectés avec de l'eau distillée ou de l'eau de pluie (pour empêcher tout changement de couleurs provoqué par la réaction des composés alcalins de l'eau du robinet avec les "substances lichéniques"). Les spécimens peuvent être pressés légèrement, puis séchés à l'aide d'un petit ventilateur afin d'accélérer le processus. On ne devrait pas utiliser la chaleur qui a souvent pour effet d'altérer les spécimens contenant des algues bleues. Les spécimens sont enfin insérés dans des enveloppes toutes faites ou fabriquées à l'aide de feuilles de 8,5 po $\times 11$ po.

Il faut également se munir d'une bonne loupe (grossissement d'environ 10 $x)$ pour examiner les échantillons, quoiqu'il soit évidemment préférable d'avoir un microscope stéréoscopique. La manipulation des plantes exige quelques bonnes lames de rasoir à un seul tranchant de même qu'une petite paire de forceps ou des pinces fines. Une aiguille encastrée dans un manche serait également commode. La plupart des caractères sont aisément repérables, qu'il s'agisse de la texture de la surface, de la présence ou de l'absence d'organes reproducteurs, ou de la coloration des faces supérieure et inférieure du thalle.

Pour tirer le meilleur parti possible de ces clefs, l'amateur doit avoir sous la main un petit flacon à compte-gouttes d'eau de Javel non dilué (p. 
ex. "Javex"), un flacon du même genre contenant une solution de lessive (environ 10 granules de lessive dans $15 \mathrm{ml}$ d'eau) ou de la potasse en solution aqueuse à $10 \%$ de même que de la paraphénylène-diamine (voir description ci-dessous). Ces produits chimiques sont utilisés pour déceler la présence de substances lichéniques propres à certaines espèces. Les tests sont effectués tout simplement en mouillant la face supérieure ou la médulle (voir fig. 65) avec une toute petite goutte de réactif. La médulle est dégagée en coupant le cortex et la couche algale qui la recouvre à l'aide d'une lame de rasoir. Moins on utilise de liquide, meilleurs sont les résultats. L'abréviation des solutions de lessive et d'hydroxyde de potassium utilisée dans les clefs est "KOH", celle de la solution d'eau de Javel "C" (symbole représentant le radical "chlorite" contenu dans le nom scientifique de l'eau de Javel, l'hypochlorite de sodium). La paraphénylène-diamine est représentée par le symbole "Pd". Si la substance lichénique recherchée est présente, il s'ensuit une réaction colorée; la partie subissant le test se teintera de jaune, d'orange ou de rouge. L'analyse au "KC" s'effectue en humectant la partie analysée avec du $\mathrm{KOH}$, puis en appliquant du $\mathrm{C}$ au même endroit. L'observation attentive des résultats s'impose, étant donné que les effets des analyses au C et au KC sont de courte durée. Les produits chimiques et les réactions à observer sont mentionnées dans les clefs quand cela est nécessaire.

Une mise en garde s'impose dans le cas de la Pd: cette substance, quoique extrêmement utile dans l'étude des lichens, peut tacher et même s'avérer dangereuse si elle est manipulée sans précaution.

Naguère, ce produit chimique était largement utilisé dans le développement des photographies, ce qui est rarement le cas aujourd'hui; par conséquent, il est difficile de s'en procurer. Pour ce faire, il est possible de commander des quantités de $100 \mathrm{~g}$ (ce qui est assez pour environ 25 ans) à un fabricant de matériel de laboratoire.

Pour obtenir une analyse à la Pd des plus réussies, dissoudre quelques cristaux du composé dans deux ou trois gouttes d'alcool ethylique (70\%). Un plat minuscule, ou une fiole, est un contenant, tout indiqué. Ensuite, à l'aide soit d'une petite brosse ( 2 ou 4 poils), soit d'une pipette très mince ou d'un compte-gouttes, mouiller la surface du lichen à analyser avec la solution de paraphénylène-diamine. La coloration apparaîtra en quelques secondes.

Préparer seulement quelques gouttes de cette solution à la fois, car elle se décompose en quelques minutes. L'expérience est vouée à l'échec si l'on utilise de la vieille paraphénylène-diamine de couleur sombre, ainsi d'ailleurs que des résidus provenant d'anciennes préparations qui se sont évaporées et qui sont remises en solution. Quant tout est fini, jeter à la 
poubelle le morceau de lichen analysé. Ceci est important étant donné que la paraphénylène-diamine tache irrëmédiablement papier, meubles, cravates, chemises, etc. et que sa manipulation sans précaution peut créer des ennuis à l'apprenti-lichénologue. Puisque c'est un poison mortel, elle doit être tenue hors de la portée des enfants.

Toute personne disposant d'un nombre de réactifs un peu plus important peut préparer une solution aqueuse et stable de paraphénylène-diamine. En premier lieu, préparer une solution à $10 \%$ de sulfite de sodium (l0 g de $\mathrm{Na}_{2} \mathrm{SO}_{3}$ dissout dans $90 \mathrm{ml}$ d'eau), ajouter quelques gouttes de détergent ("Photo-flo", fabriqué par kodak, est le meilleur) et puis $1 \mathrm{~g}$ de paraphénylène-diamine. Brasser et agiter le mélanger pendant quelques minutes jusqu'à ce que la majeure partie de la substance soit dissoute. Puis filtrer la solution saturée et se débarasser des cristaux non dissous. La solution rose pâle ainsi préparée est relativement sensible et se conservera un mois et même davantage (surtout dans une bouteille de couleur sombre). Elle demeure toxique et susceptible de tacher les objets; aussi les précautions d'usage s'avẽrent-elles toujours indispensables lors de la manipulation de la solution. Une fois rose foncé, elle doit être jetée.

\section{Les lichens crustacés}

Les lichens crustacés ne passent pas toujours inaperçus, bien qu'il faille reconnaitre que certains d'entre eux sont à peine plus que des taches noires. Il y en a qui arborent de grands thalles ou des fructifications aux couleurs vives et qui envahissent les rochers, l'écorce ou le sol. Ils suscitent un intérêt tout particulier en raison du lien étroit qui les unit à leur "hôte" ou substratum; ils poussent fréquemment non seulement à sa surface mais aussi à l'intérieur de celui-ci. Il est bien connu que les lichens crustacés qui colonisent le calcaire ou le granite sont incorporés au roc; en effet ils s'infiltrent entre les veines et les cristaux et atteignent plusieurs millimètres de profondeur. Les tissus de bon nombre d'espèces corticoles se développent sous le liège. Fait révélateur, ces lichens crustacés ont, dans une proportion importante, tendance à être liés à un substratum précis, c'est-à-dire à se retrouver sur une seule espèce d'arbre ou tout au plus chez un nombre restreint d'essences. Par contre, d'autres semblent s'installer partout; il y a, par exemple, des lichens de toundra qui, au début, vivent sur le sol et poursuivent leur développement sur des végétaux morts, des mousses, voire des rochers. Toutefois ces espèces à croissance rapide sont l'exception qui confirme la règle, la plupart des autres lichens crustacés grandissant lentement, surtout dans l'Arctique. Dans ces régions, la plaque de grandeur moyenne d'un lichen crustacé habitant une pierre, peut 
donc être âgée de plusieurs centaines d'années et, dans des cas bien particuliers, l'âge du thalle a été estimé à plus de mille ans.

Étant donné que les lichens crustacês sont totalement fixés au support, il faut détacher un morceau du substrat lors de la récolte. Celle des espèces corticoles ne soulève aucune difficulté particulière puisqu'il est aisé de détacher l'écorce; de leur côté, les lichens saxicoles présentent un petit problème. La plupart des lichénologues optent pour un petit ciseau à froid ( $\frac{1}{2}$ - $\frac{3}{4}$ pol et un marteau de minéralogiste pour dégager les thalles crustacés. Le botaniste quelque peu expérimenté, armé d'un ciseau bien aiguisê et de bonne volonté, viendra à bout de faire de la plupart de ces plantes des spécimens convenables; cependant je suis bien obligé d'avouer mon impuissance devant ces sacrés thalles crustacés (toujours les plus intéressants) qui habitent les parois rocheuses lisses.

Bien que les lichens soient un complexe formé de deux plantes, un champignon et une algue, c'est uniquement du premier dont on se sert dans la classification. Lors de la dêtermination des lichens, nous devons donc nous pencher surtout sur les champignons, et en particulier sur leurs fructifications. Dans la région de l'outaouais, tous les lichens appartiennent à une classe de champignons appelée Ascomycètes. Tous produisent des spores dans de petits sacs claviformes ou cylindriques, appelés asques (fig. 71). Chaque asque renferme ordinairement huit spores, bien que leur nombre puisse aller de quelques-unes à plusieurs centaines. En général, les asques prennent naissance ensemble dans un tissu spécial l'hyménium (fig. 70), en même temps que des filaments stériles (paraphyses). Cet hyménium, de même que d'autres tissus vitaux, se trouve à l'intérieur de divers types de fructification: ce sont normalement des structures cupuliformes ou discoïdes appelés apothécies (fig. 65 et 74 a) ou des parties plus ou moins enfoncées et urcéolées nommées périthèces (fig. 69, 74c). Comme on le verra, il existe êgalement d'autres genres de fructifications.

L'utilisation de cette clef requiert un microscope composé. Il permet de se baser sur les caractères des spores, rendant par le fait même la détermination relativement aisée, rapide et sûre. Puisque la clef nécessitera rarement l'observation d'autre chose que la forme, la taille et la couleur des spores de même que le type de cloisonnement (septation), un grossissement 100 $\mathrm{X}$ sera souvent amplement suffisant et un ajustement à $400 \mathrm{x}$ constituera la limite supérieure. Il est certain que l'utilisation du microscope sans trop d'efforts et avec efficacité repose sur la qualité de la source lumineuse, la propreté de la lentille ainsi que la malléabilité de la crémaillère et de la vis micrométrique.

L'examen des spores nécessite des préparations fructifications pour le 
microscope. Pour commencer, il s'agit d'avoir près de son microscope les quelques objets suivants: un flacon à compte-gouttes contenant de l'eau, un autre renfermant un peu de $\mathrm{KOH}$ (la même "recette" que dans le cas des analyses de couleurs), une grosse aiguille à coudre encastrée dans un manche de bois et un paquet de lames de rasoir à un seul tranchant.

Le montage de la préparation pour le microscope consiste, dans un premier temps, à simplement mouiller l'organe reproducteur d'une goutte d'eau et, une fois le tissu imbibé et ramolli, il s'agit de pratiquer quatre ou cinq coupes verticales à travers le coeur de la fructification. Si cette opération est effectuée à l'aide d'une loupe, ou mieux encore, d'un microscope stéréoscopique (à environ $12 \mathrm{x}$ ), il est possible d'obtenir des coupes étonnamment minces avec très peu d'expérience. Cela se révèle particulièrement vrai avec des lames de rasoir neuves. Il ne faut pas détacher la fructification, car cela facilite la coupe si elle demeure en place.

Une fois cette dernière terminée, prendre les coupes sur le bord du rasoir et les déposer dans une petite goutte d'eau sur une lame de microscope propre. Avec un peu de chance, l'une des coupes au moins sera assez mince pour être examinée. Si elles ne flottent pas librement dans la goutte d'eau, c'est sans doute qu'elles sont fixées à la base. Recouvrir alors les coupes avec la lamelle et la déplacer doucement d'avant en arrière de sorte qu'elles se libèrent et s'étendent.

Il y a ordinairement assez de spores qui s'échappent de l'hyménium tranché sans qu'il y ait besoin de plus de manipulations pour procéder facilement à leur examen. Néanmoins, si elles s'obstinent à demeurer à l'intérieur des asques, le naturaliste, tout en regardant dans son microscope à une faible puissance $(100 \mathrm{X})$, exerce un pression sur la lamelle, avec le côté de l'extrẻmité de l'aiguille, à proximité de l'endroit de l'hyménium plein de spores. Cela permet de voir réellement les spores se dégager des asques. C'est également la meilleure façon de dissêminer les asques, ce qui permet de compter le nombre de spores contenues dans chacun d'eux. Dans le cas d'un hyménium récalcitrant (chez certaines espèces, il ne s'écrase pas facilement), ajouter une goutte de $\mathrm{KOH}$ au bord de la lamelle et l'amener en dessous en absorbant l'eau sous la lamelle du côté opposé avec un papier-mouchoir ou un morceau d'essuie-tout. Cela dissoudra la substance qui maintient les asques ensemble à l'intérieur de l'hyménium. Une autre tentative d'écrasement et le tour est joué!

\section{REMERCIEMENTS}

Il me fait plaisir de mentionner le dévouement de mon prëcieux 
collaborateur, Pak Yau Wong, dont l'aide au cours de bon nombre de determinations, la mise à l'essai des premières esquisses de clefs et les récoltes de grande valeur ont été extrêmement utiles. Anne Hanes, qui a dessiné les figures 6, 9, 10 et 68-74, a également livré ses commentaires sur les clefs parues dans Trail \& Landscape et a ajouté un grand nombre de faits à la flore. Susan Laurie-Bourque a exécuté toutes les illustrations, exception faite de celles mentionnées ci-haut et des figures 54, 57, 58, 60, et 62-67, qui ont été dessinées par Brenda Carter. Quant à la version française, elle a été prêparée avec soin par sylvie Lemieux. Je voudrais remercier tous ces gens, ainsi que mes amis, mes collègues et les nombreux étudiants qui ont utilisé les premières clefs et qui ont révélé avec délicatesse les erreurs qu'ils y avaient décelées. 


\section{LES GENRES CRUSTACÉS (ÉCHANTILLONS FERTILES)}

1. Fructifications portées sur un petit pied filamenteux de $1 \mathrm{~mm}$ de hauteur (fig. 6); spores généralement libres (c.-à-d. non contenues dans des asques) et massées dans des structures cupuliformes. Thalle installé sur les vieilles souches et les chicots d'arbre ou, de temps en temps, sur l'ecorce . . . Caliciales

1. Fructifications dépourvues de pied: elles sont soit fixées directement au thalle, soit enfoncées à l'intérieur de celui-ci . . . . . . . . . . . . . . . . • • . . . . . 2 .

2. Thalle gelatineux une fois mouillé, noir, renfermant des algues bleues, spores non cloisonnées; bord de l'apothécie contenant des algues . . . . . . . . . . . . . . 3 .

2. Thalle non gëlatineux au contact de l'eau; il est constitué d'algues vertes sauf chez les Placynthium, dont les apothécies sont lécidéines (pas d'algues) et les spores cloisonnées, et chez les Pannaria, dont le thalle est squamuleux (v. doublet 36) . . . . . . . . . . 4.

3. Thalle membraneux dont les algues sont agglutinées en chapelet (Nostoc); apothécies plus ou moins à l'intérieur du thalle ou des crêtes thallines ( $v$. clef des espèces foliacẻes, doublet 30 ). Taxons rares, terricoles ou mussicoles . . . . . . . . . . . . . . . . . . . . Lempholemma

3. Thalle granuleux, dont les algues forment des nodules serrés de quelques cellules (GLoeocapsa); apothécies presque globuleuses, superficielles, ressemblant d'abord à un périthèce, puis s'ouvrant graduellement au sommet, ce qui leur confère une apparence lécanorine; spores: 12-14 x 6-7 $\mu \mathrm{m}$. Espèce rare; calcicole.. . . . . . . Psorotichia schaereri

4. Thalle ou organes reproducteurs jaune vif, orange ou

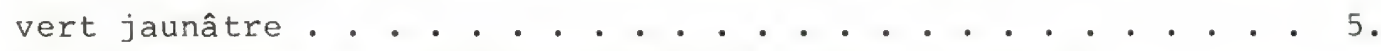

4. Thalle et organes reproducteurs gris, bruns, noirs ou verts, dépourvus de pigments jaunes (quoique certaines espèces déterminées ici auront des apothécies jaunâtre

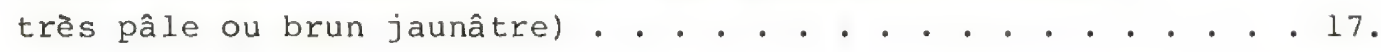


5. Thalle corticole ou lignicole . . . . . . . . . . . . . . . . 6 .

5. Thalle saxicole . . . . . . . . . . . . . . . . . . 1 . .

6. Thalle ou apothẻcies $\mathrm{kOH}+$ rouge violacé sombre; spores polariloculaires (fig. 72) . . . . . . . . . . Caloplaca

6. Thalle et apothécies KOH- . . . . . . . . . . . . . . . 7 .

7. Thalle vert jaunâtre pâle (présence d'acide usnique) ou vert-de-gris; couleur des apothécies variant entre brun jaunatre et jaune vif; spores incolores, au nombre de 8 par

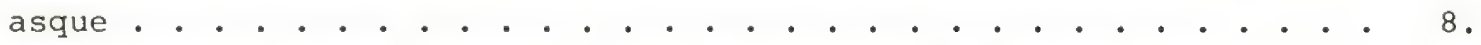

7. Thalle d'un ton vif de jaune d'oeuf ou vert jaune brillant. . . . . 9.

8. Spores unicellulaires, ellipsoïde ou ayant la forme d'une ellipse légèrement allongée; apothécies jaunâtre . Lecanora 8. Spores cloisonnées, ayant la forme d'un fuseau étiré; spores orange pâle . . . . . . . . . . . . . . . Dimerella

9. Thalle aréolé (fig. 68) ou grossièrement granuleux

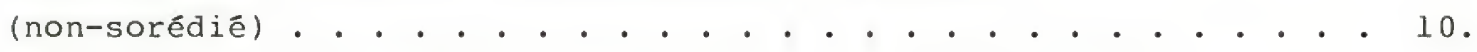

9. Thalle couvert de sorédies granuleuses, jaune vif; spores incolores, unicellulaires, petites, ellipsoides, au nombre de 16 par asque... . . . . . (fig. 3) Candelariella efflorescens

10. Fructifications jaunes, discoïdes (apothécies typiques); thalle composé de grosses granulations jaunes ou de petites aréoles; spores incolores, unicellulaires, fréquemment au nombre de 16 par asque . . . . . . Candelariella

10. Fructifications noires, constituées en réalitê d'une masse de spores en liberté à l'intérieur d'une sorte de "coupe", enfoncées dans les aréoles d'un thalle vivement coloré de jaune ou de vert jaunâtre; spores brun foncé, bicellulaires, non contenues dans des asques. Espèce peu fréquente, habitant les clôtures de bois et les souches de conifères .. . . . . (fig.7) Cyphelium tigillare

11. Thalle au pourtour nettement lobé, dont le cențre devient

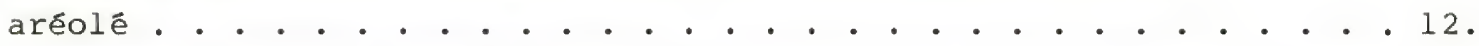

11. Pourtour du thalle pas du tout lobé . . . . . . . . . . . . . . 14 . 

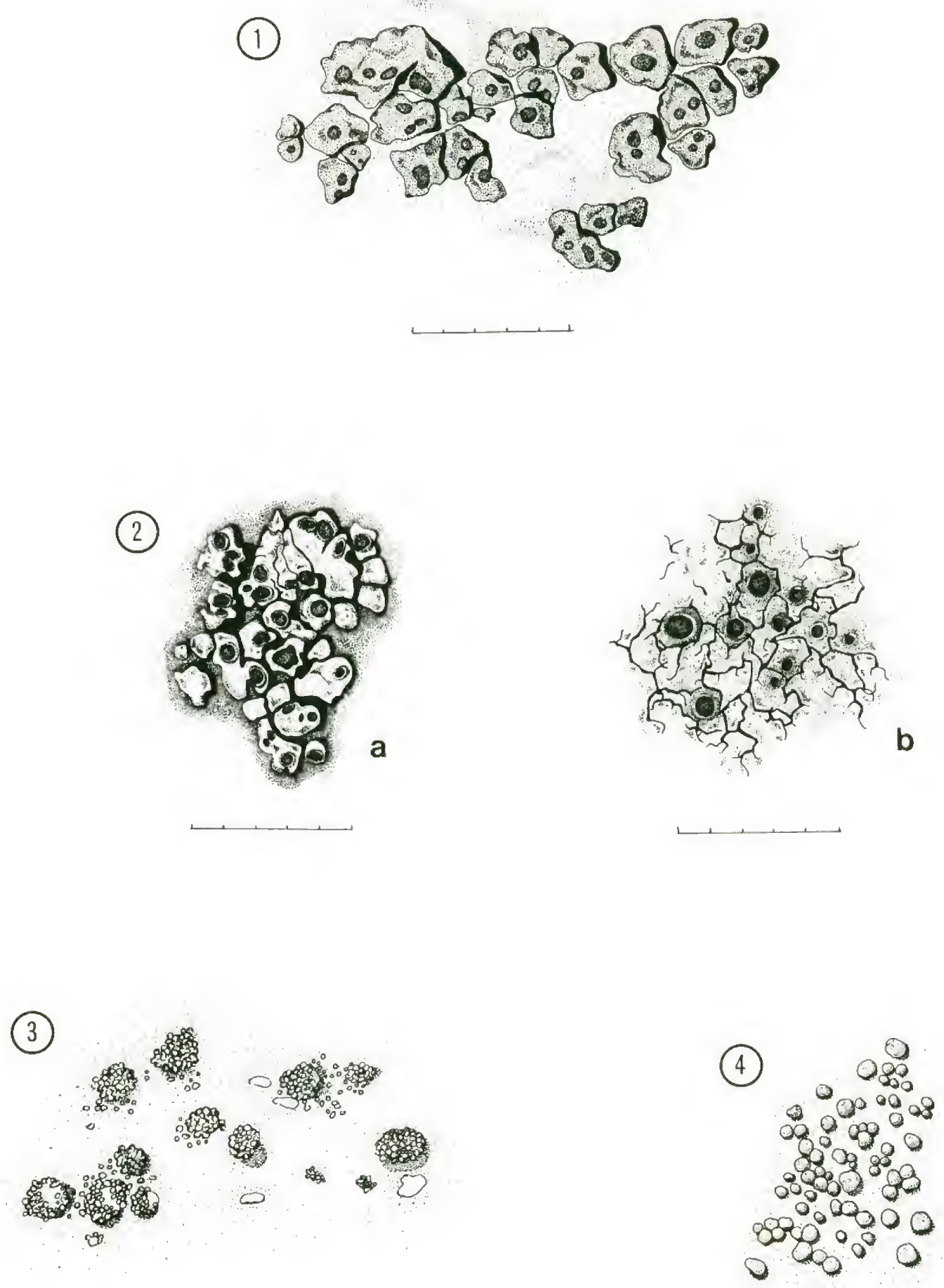

Figures 1 à 4.-1. Acarospora fuscata. -2. Aspicilia cinerea: a) thalle fendillé-aréolé; b) thalle continu par endroits et fendillé ailleurs. -3. Candelariella efflorescens. -4. Candelariella xanthostigma. Échelle: chaque division $=1 \mathrm{~mm}$. 
12. Thalle vivant sur les roches siliceuses, p. ex. le granite et le gneiss . . . . . . . . . . . . . . . . 13.

12. Thalle se trouvant ordinairement sur des roches calcaires, p. ex. le calcaire; apothécies brun jaunâtre pâle; spores incolores, unicellulaires. Espèce commune. . . . . . . . . . . . . . . Lecanora muralis

13. Apothécies variant entre brun foncé et noir; spores brun foncé, bicellulaires. Espèce peu fréquente

- (fig. 22) Dimelaena oreina

13. Apothécies allant de jaunâtre ou brun rougeâtre à roux; spores incolores, unicellulaires . . . . . . . . . . . . . Lecanora

14. Thalle ou apothécies $\mathrm{koH}+$ rouge violacé . . . . . . . . . . . 15.

14. Thalle et apothécies $\mathrm{KOH}-$ ou $\mathrm{KOH}+$ jaune (pas rouge violacé) . . . . . . . . . . . . . . . . . . . . 16.

15. Spores unicellulaires; apothécies convexes, dont la marge disparaît. Espèce rare; habitant les roches calcaires

15. Spores polariloculaires (fig. 72d); apothêcies généralement planes, entourées d'un bord distinct, persistant

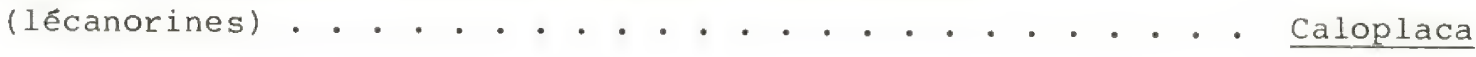

16. Spores unicellulaires, incolores, apothécies lécanorines et jaunes . • • . . . . . . . . . . . . . . . . Candelariella (N.B.: Le lichen aquatique, orange pâle, Hymenelia lacustris, peut ëgalement être identifié ici. Se reporter à la clef des Aspicizia.)

16. Spores murales (fig. 72a), brunes ou incolores; apothêcies lêcidêines, noires ou roux sombre

Rhizocarpon

17. Fructifications de forme irrégulière, surtout allongées et souvent ramifiées, jamais immergées dans le thalle (fig. 9). Toutes les espèces de la région outaouaise sont corticoles ou lignicoles . . . . . . . . . . . . . . . . . . . . . 18 .

17. Fructifications maculiformes, discoïdes, cupuliformes ou verruciformes, ni étirées, ni irrégulières, exception faite de quelques-unes qui sont immergées dans le thalle... . . . . . 21 .

18. Spores murales (fig. 72b) très grandes; taxons peu communs . . . . . . . . . . . . . . . . . . Arthothelium 
18. Spores seulement cloisonnẻes transversalement (fig. $72 \mathrm{e}$

à h) contenant de 2 à 14 cellules . . . . . . . . . . . . . . . 19.

19. Cellules sporifères lenticulaires (fig. 72h), spores

d'incolores à brun très clair, contenant de 6 à 14 cellules;

fructifications striêes d'une fissure distincte, étroite ou

large, dans le sens de la longueur, mais pourvues de parois toujours nettes et noires. Espèce fréquemment observée sur différents arbres . . . . . . . . . . (fig. 9) Graphis scripta

19. Cellules sporifères "carrées" (c.-ã-d. cylindriques fig. 72g) . . . . . . . . . . . . . . . . . . . . . . 20.

20. Fructifications proêminentes, s'ouvrant habituellement par une fissure bien nette; paroi noire, carbonacée et épaisse; nombre de cellules par spore variant de 4 à 6 . Espèce rare; corticole ou lignicole . . . . Opegrapha pulicaris (N.B.: Les champignons qui appartiennent au genre Hysterium ressemblent énormément à opegrapha spp.)

20. Fructifications aplaties (pas proéminentes), ne s'ouvrant pas par une fissure; absence de paroi externe

..................... (... (fig. 10) Arthonia

21. Fructifications au moins partiellement enfoncées dans le thalle. . . . . . . . . . . . . . . . . . . . 22.

21. Fructifications superficielles, qui ne sont pas ordinairement enfoncées dans le thalle (bien qu'elles soient parfois insérées entre les aréoles; v. fig. 68) nettement discolides ou cupuliformes, de temps en temps convexes ou même hêmisphériques (fig. $68,74 \mathrm{a}$ ) . . . . . . . . . . . . . . . . . . 42 .

22. Apothêcies enterrées sous un amas ou une couche de sorédies . . . . . . . . . . . . . . . . . . . . . . . . . 23.

22. Apothécies non enterrées sous un amas ou une couche de sorédies. . . . . . . . . . . . . . . . . . . . 24 . .

23. Spores murales, une par asque; thalle $\mathrm{KOH}+$ rouge (acide norstictique). Espèce rare; corticole . . . . . . . Phlyctis argena

23. Spores non cloisonnées, une ou deux par asque (fig. $7 \mathrm{lc}$ ); thalle $\mathrm{KOH}_{-}$- ou KOH+ jaune . . . . . . . . . . . . . . Pertusaria 
24. Fructifications s'ouvrant à la surface par une ouverture plus ou moins large, s'apparentant parfois à une ostiole; vu en coupe, l'organe reproducteur n'est pas urcéolé, ni pourvu d'une paroi noire et carbonacée (sauf chez Acarospora badiofusca); spores incolores, non cloisonnées . . . . . . . . . . . . . . . 25.

24. Fructifications ordinairement urcéolées, apparaissant sous la forme d'une saillie pointue, d'une petite plaque ronde ou d'un minuscule "cratère" sous le microscope; elles s'ouvrent vers 1 'extérieur par un petit pore ou ostiole (fig. $26,69,74 \mathrm{~b}$ à d) et sont partiellement ou totalement enveloppés par une paroi noire et carbonacée . . . . . . . . . . . . . . 27.

25. Fructifications enfoncees dans une "verrue" thalline, se retrouvant plus d'une par verrue habituellement (fig. 24, 74b); très grosses spores unicellulaires, à paroi épaisse ordinairement, dont le nombre varie le plus souvent entre 2 et 4 à l'intérieur d'un asque (fig. 71c) . . . . . . Pertusaria

25. Les fructifications ne sont pas situées à l'intérieur de verrues, mais sont bien plutôt enfouies dans le thalle . . . . . 26 .

26. Très petites spores au nombre de 32 ou même davantage par asque; thalle dispersé-aréolé (fig. 1), ordinairement brun, parfois pruineux . . . . . . . . Acarospora

26. Spores au nombre de 8 par asque; thalle continu, lisse à fendillé-aréolé ( $\mathrm{f}$ ig. 2), ordinairement gris, jamais pruineux . . . . . . . . . . . . . . . Aspicilia

27. Spores murales... . . . . . . . . . . . . . . 28 .

27. Spores allant d'unicellulaires à pluricellulaires, non murales . . . . . . . . . . . . . . . . 32 .

28. Espèces saxicoles ou terricoles . . . . . . . . . . . 29.

28. Espèces corticoles. Thalle extrêmement mince, voire absent . . . . . . . . . . . . . . 31.

29. Thalle légèrement brun, lisse, épais, C-; fructifications (périthèces) qui s'ouvrent par un pore minuscule (fig. 74c); spores brunes habituellement, très grandes, 2 à 6 par asque; petites cellules algales dans 1 'hyménium au milieu des asques (fig. 26) . . . . . . . . . . . . . 30 . 
29. Couleur du thalle variant entre gris et blanc, celui-ci étant aréolé, très épais, C+ rouge; fructifications ouvertes par un cratère profond, souvent large, qui donne dans la cavité hyméniale (fig. 74d). Très commun; sur les rochers secs, ensoleillés et exposés de même que sur le sol avoisinnant

\section{Diploschistes scruposus}

30. Thalle squamuleux, surface disjointe, uniforme ou entre les deux; périthèces enterrés sous les squamules, minuscules ostioles noirs, visibles à la surface. Rare; sur le calcaire... . . . . . . . . Endocarpon pusillum

30. Surface du thalle uniforme, fendillée-aréolée ou entre les deux; périthèces partiellement exposés parfois. . Staurothele

31. Fructifications divisées en un certain nombre de cavités, chacune d'elles s'ouvrant par son propre ostiole; spores hyalines, brunes ou entre les deux, 30-48(-51) x (12-) 15-18(-21) um (Harris, 1973). [Le spécimen de l'Outaouais était dépourvu de spores.] Rare; qui pousse sur les arbres à feuilles caduques, surtout le chêne et l'érable

31. Fructifications à une seule cavité; spores incolores (fig. 72d). Peu abondant, qui pousse surtout sur le bouleau blanc, mais très difficile à voir et sans doute négligé pour cette raison . . . . . . . . . . . Polyblastiopsis fallaciosa 32. Espèces saxicoles ou terricoles . . . . . . . . . . 33 . 32. Espèces corticoles . . . . . . . . . . . . . . 35.

33. Espèces terricoles. Spores unicellulaires, ellipsoïdes un

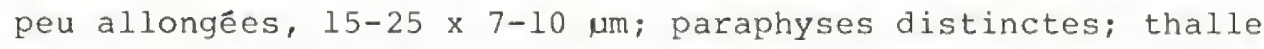
formant une croûte membraneuse et verte sur le sol nu, avec les périthẽces apparaissant comme des petits points noirs. Rare . . . . . . . . . . . . . . . . Thrombium epigaeum

33. Espèces saxicoles. Spores unicellulaires ou bicellulaires; paraphyses non distinctes . . . . . . . . . . . . . . 34 .

34. Spores unicellulaires, ellipsoïdes; thalle mince ou épais . . . . . . . . . . . . . . . . . Verrucaria 
34. Spores bicellulaires, ellipsoïdes, 22-30(-33) x 14-17 (-20) $\mu \mathrm{m}$; couleur du thalle variant entre gris et blanc, celui-ci étant épilithique ou endolithique; cratères creusés par des périthèces dans la roche. Rare; qui pousse sur le calcaire. . . . . . . . . . Thelidium decipiens

35. Spores qui peuvent atteindre 36 cellules, celles-ci sont incolores, très longues et filiformes et se rompent parfois en segments plus petits (fig. 72f); fructifications noires, légèrement surélevées et ouvertes par un cratère profond; le thalle s'étale en plaques blanches sur les érables à sucre ou, rarement, sur d'autres essences à feuilles caduques. Commun . . . . . . . . . . . . . . . . . Conotrema urceolatum

35. Spores qui ont jusqu'à 7 cloisons, forme des spores variable: fuseau étroit, ellipse ou forme intermédiaire; pore des fructifications petit et peu visible en général (pas de

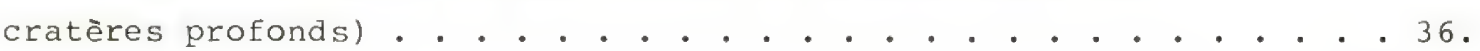

36. Quelques fructifications (pêrithèces), chacune ouverte par un ostiole, agglomérées de façon à former une verrue, les verrues sont ellipsoïdes, allongées ou entre les deux et mesurent jusqu'à $3 \mathrm{~mm}$ de longueur; spores incolores, 7 à 9 cloisons, cellules polygonales, lenticulaires ou entre les deux (comme chez Graphis, fig. 72h), 38-52 x 7-10 um (Harris, 1973). [Les spécimens de la région de l'Outaouais sont stériles habituellement.] Rare; croît sur le hêtre ou, exceptionnellement, sur d'autres essences à feuilles caduques . . . . . . . . . . . . . . . Trypethelium virens

36. Fructifications isolées, pas agglomérées de façon à

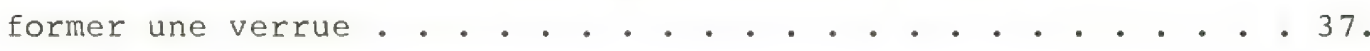

37. Ostiole des fructifications placé non pas au sommet, mais sur le côté, qui se termine par une sorte d'encolure montante; diamètre des fructifications $0,5-0,8 \mathrm{~mm}$; spores incolores ou brunes, 3 - 7 cloisons. Rare; vit sur les arbres à feuilles caduques, l'érable et le frêne surtout . . . . . . . . . . . Plagiocarpa

37. Ostiole des fructifications central, au sommet, peu visible souvent; diamètre des fructifications $0,1-0,8 \mathrm{~mm}$. . . . . . . . 38 . 


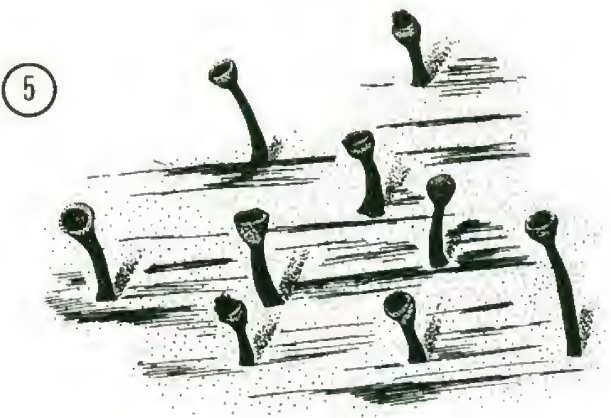

(6)
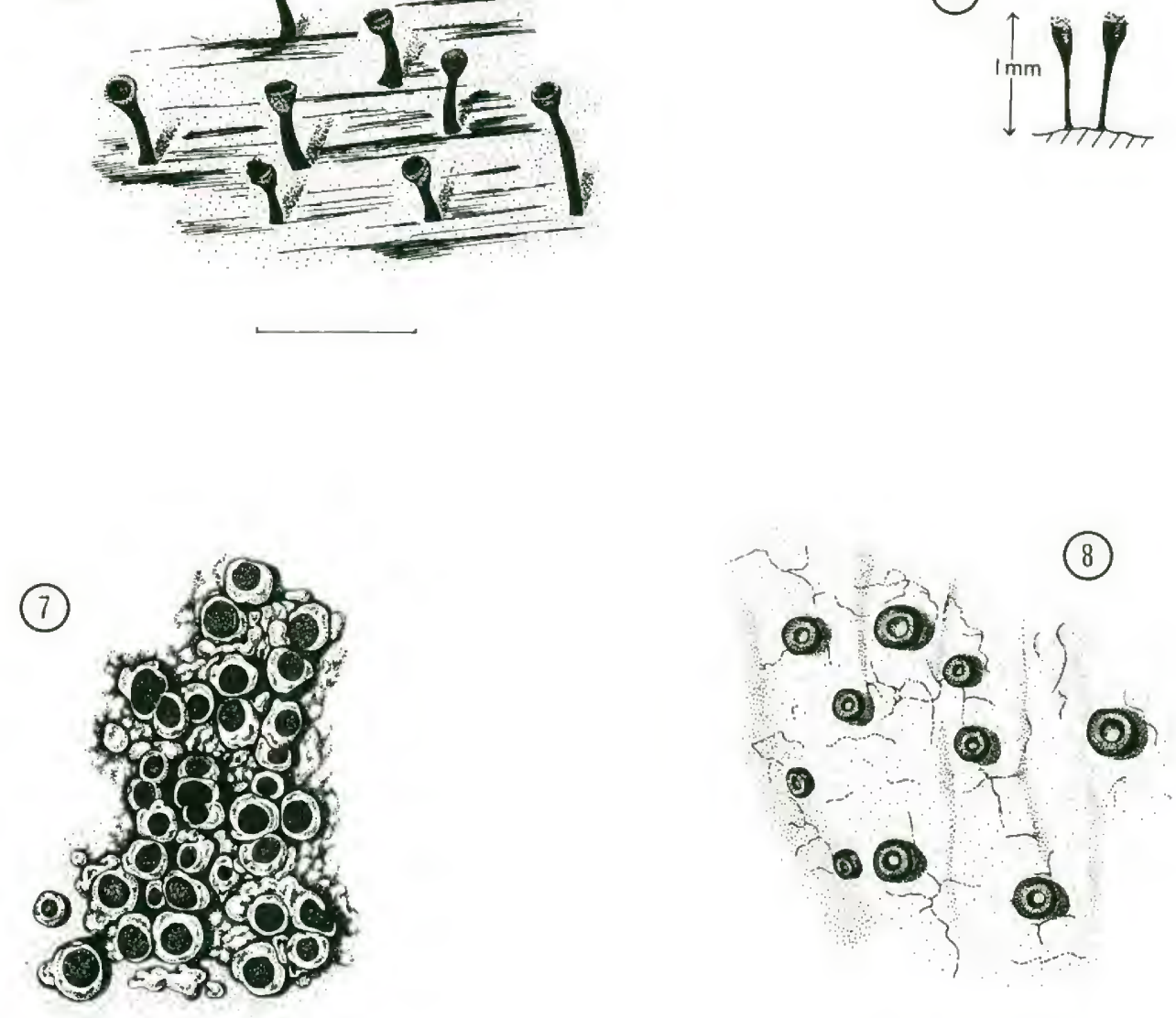

(9)

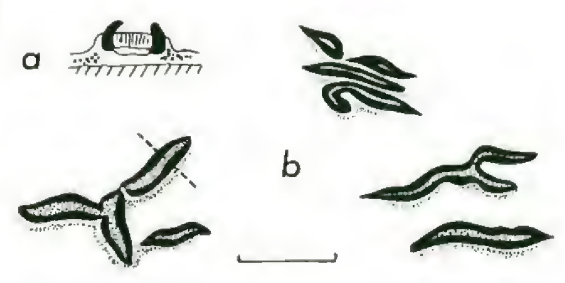

(10)

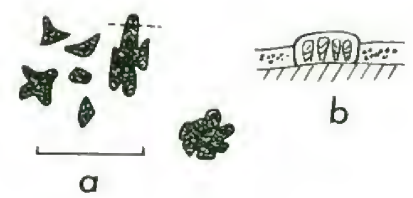

Figures 5 à 10.-5. Calicium trabinellum. -6. Mycocalicium parietinum. -7. Cyphelium tigillare. -8. Conotrema urceolatum. -9. Graphis scripta: a) coupe verticale d'un ascocarpe (v. ligne pointillêe); b) ascocarpes allongés (apothécies). -10. Arthonia radiata: a) ascocarpes; b) coupe verticale d'un ascocarpe (v. ligne pointillée en "a") illustrant les caractères des Ascoloculaires. Échelle: chaque division $=1 \mathrm{~mm}$. 
38. Fructifications de $0,1-0,3 \mathrm{~mm}$ de diamètre, minuscules; algues dans le thalle parfois; cellules sporifères cylindriques ou lenticulaires . . . . . . . . . . . . . . 39.

38. Fructifications de 0,3 - 0,8 mm de diamètre; le thalle renferme toujours des algues (Trentepohlia); cellules sporifères lenticulaires . . . . . . . . . . . . . 4 4 .

39. Spores brunes, 5 - 6 cloisons, 18-24 x 5-9 um (Harris, 1973), cellules lenticulaires; périthèces d'environ 0,2 - 0,4 mm de diamètre; base de la paroi du périthèce pâle; thalle blanc, hypophleode. Rare; crô̂t sur les arbres à feuilles caduques, l'érable surtout . . . . . . . . . . . . . Eopyrenula leucoplaca

39. Spores incolores, 0 - 3 cloisons, cellules cylindriques; diamètre des fructifications $0,1-0,25 \mathrm{~mm}$. . . . . . . . . . . . 40.

40. Espèce qui s'installe sur le bouleau blanc, plaque pâle, à peine visible sur l'écorce blanche; spores en forme de fuseau étroit, 20-30 x 2-4 um (Harris, 1973), en forme de banane (lēgè rement courbe), une ou deux cloisons sont parfois visibles. Fréquent, mais très difficile à voir . . . . . . . . . . . . . . . . Leptorhaphis epidermidis

40. Espèce qui pousse sur d'autres essences. Spores à $1(-3)$ cloisons, fusiformes, droites, 15-20 x 4,5-6,0 $\mu \mathrm{m}$ (Harris, 1973) . . . . . . . . . . Arthopyrenia s. lat.

41. 5 - 6 cloisons dans les spores; diamètre des périthèces: 0,2 - 0,4 mm; ostioles concaves, bien visibles (voir le doublet 39) . . . . . . . . . . . . . . . . Eopyrenula leucoplaca

41. Spores à 3 cloisons; diamètre des périthèces: $0,3-0,8 \mathrm{~mm}$, ostiole à peine visible, non concave . . . . . . . . . . . . Pyrenula

42. Algues bleues (couleur de la couche algale variant entre vert-de-gris foncé et vert bleuâtre) . . . . . . . . . . . . 43. 42. Algues (et la couche algale) vert tendre . . . . . . . . . . 44.

43. Thalle composé de squamules brunâtres, imbriquẻes et lobées; apothécies tantôt lécanorines, tantôt lécidéines. Peu frëquent; saxicole, vit habituellement dans des stations ombragées ou humides . . . . . . . . . . . . . Pannaria leucophaea 
43. Thalle presque noir, grossièrement granuleux ou isidié, ou encore entre les deux, qui devient aréolé; habituellement bordé d'une marge noir bleuâtre, bien visible; spores à une cloison, apothécies noires, lécideines. Fréquent; pousse sur toutes sortes de roche, mais surtout sur les roches calcaires... . . . . . . . . . . . placynthium nigrum

44. Présence d'algues dans les bords et sous l'hyménium des fructifications (fig. $70 \mathrm{~b}$ ) . . . . . . . . . . . 45 .

44. Absence d'algues dans les fructifications (fig. 70a) . . . 52 .

45. Fructifications à l'intérieur de saillies ou verrues sorédiêes, situées sur le thalle. . . . . . . . . . . . . . . 46.

45. Fructifications non situées dans des saillies sorédiées . . . . . 47.

46. Spores murales, incolores, une par asque (v. doublet 23) . . . . . . . . . . . . . . . . . . . Phlyctis argena 46. Spores unicellulaires, incolores........ . Pertusaria

47. Enormes spores, une par asque . . . . . . . . . . . . Pertusaria

47. Nombre de spores par asque variant entre 4 et 8 (fig. 71a). . . . 48.

48. Spores unicellulaires, ellipsoïdes . . . . . . . . . . 49.

48. Spores bicellulaires ou pluricellulaires, ellipsoïdes ou vermiformes . . . . . . . . . . . . . . . 50.

49. Largeur des apothécies atteignant $3 \mathrm{~mm}$, ces dernières étant pourvues de marges très épaisses; spores assez grandes, dont la longueur dépasse $30 \mu \mathrm{m}$, ellipsoïdes; disques légèrement teintés de rose normalement . . . . . . . . . . . Ochrolechia

49. Largeur des apothécies n'atteignant pas, en règle générale, $1,5 \mathrm{~mm}$; longeur des spores inferrieure à $20 \mu \mathrm{m}$; coloration des apothécies variant entre brun clair et brun foncé ou noir . . . . . . . . . . . . . . . . . . . . . Lecanora

50. Spores incolores... . . . . . . . . . . . . 51.

50. Spores brunes foncées; parois des spores d'épaisseur souvent variable ( $\mathrm{fig} .72 \mathrm{~m}$ ), mais parfois égale (fig. 721); apothécies à disque brun très foncé . . . . . Rinodina 
51. Spores ellipsoïdes, bicellulaires (la plupart se trouvent à l'intérieur d'apothécies de tout genre); thalle et bords de l'apothêcie $\mathrm{koH}-$. . . . . . . . . . . . . . . . . . . Lecania

51. Spores fusiformes, enroulees et courbees, plus ou moins cloisonnées souvent; thalle épais, verruqueux, $\mathrm{koH}+$ jaune vif (acide thamnolique); disque des apothécies roux, pruineux parfois, qui peut mesurer jusqu'à $2 \mathrm{~mm}$ de diamètre; le bord des apothécies semble se dédoubler souvent, la partie supérieure étant quelque peu déchiquetée. Rare; croît sur l'écorce, particulièrement sur celle du thuya occidental

. . . . . . . . . . . . . . . . Haema tomma ochrophaeum

52. Spores unicellulaires . . . . . . . . . . . 53 .

52. Spores bicellulaires ou pluricellulaires . . . . . . . 56.

53. Multitude de petites spores à l'intérieur de chaque asque (ce qui confère souvent à l'asque mûr un aspect grossièrement granuleux, fig. 7lb) apothécies noires ou pruineuses; thalle souvent presque absent (endolithique). Lichens saxicoles. . Sarcogyne

53. Spores en groupe de 8 dans chaque asque . . . . . . . . . . . 54.

54. Thalle entièrement constitué de grosses granulations vertes ou sorédies granuleuses; fructifications presque noires, dépourvues de rebord propre; thalle ct rouge. Espèce rare; se retrouvant sur le bois tendre en décomposition . . . . . . . . . . . . Micarea viridescens

54. Thalle jamais entièrement sorédié; fructifications

(apothécies) ordinairement pourvues d'un rebord distinct, (fig. 70c) quoique, chez certaines espèces, les vieilles apothêcies soient immarginées . . . . . . . . 55.

55. Thalle squamuleux................ Psora s. $1 .$.

55. Thalle continu, arêolé, ou entre les deux, pas squamuleux, parfois peu visible ou très mince. . . . . . . . . Lecidea $\mathrm{s}_{\text {. }}$.

56. Spores murales... . . . . . . . . . . . . 57.

56. Spores non murales, cloisonnées transversalement seulement . . . . . . . . . . . . . . . 58. 
57. Espèce corticole (habituellement sur le thuya occidental); apothêcies noires, énormes spores incolores, pluricellulaires, une par asque; thalle vert olive, souvent en compartiments ou aréoles. Espèce rare(?) . . Lopadium pezizoideum (N.B.: C'est M. John Macoun qui a signalé cette espèce dans la région outaouaise, mais il n'y a aucun spécimen d'herbier au CANL.)

57. Espèces de roches non calcaires, apothécies noires ou brunes foncées, habituellement insérées entre les aréoles (rarement enfoncées dans ces dernières) (fig. 68); spores incolores à vertes foncées ou brunes, 8 par asque . . . . . . . . . . . Rhizocarpon

58. Spores brunes foncées, bicellulaires; apothécies noires • Buellia 58. Spores incolores . . . . . . . . . . . . . . . . 59.

59. Rebord propre distinct, à disposition radiale (v. fig. $70 a$ et 74 a); paraphyses simples ou peu ramifiées, dont l'extrémité est ordinairement clairement épaissie (fructifications

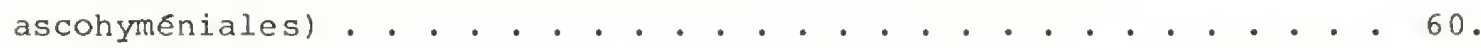

59. Rebord propre peu distinct ou absent, dont la disposition n'est jamais radiale (fig. lob, v. en coupe); filaments entre les asques très ramifiés et anastomosés, dont l'extrémité n'est pas épaissie (fructifications ascoloculaires) . . . . . . . . 64.

60. Spores formées de (2), 4 ou 16 cellules.......... . . . 61 . 60. Spores invariablement bicellulaires . . . . . . . . . . . 62 .

61. Spores (surtout les jeunes) entourêes d'un épais "halo" clair et gêlatineux, ou épispore, situé à l'extérieur de la paroi normale de la cellule (fig. 72a); spores bicellulaires ou quadricellulaires, forme variant entre celle d'une ellipse et celle d'un court fuseau. Lichens rares; saxicoles. . . . Rhizocarpon

61. Spores dépourvues d'épispore gêlatineuse, dont l'aspect varie de fusiforme à aciculaire, par 3-16 . . . . . . . . . . . Bacidia

62. Couleur du thalle variant entre rose pâle et brun cuivré; asques longs et élancés; forme des spores variable: fusiformes à presque aciculaires. Lichens corticoles; rares . . . . . . . . . . . . . . . Dimerella 
62. Apothécies noires ou brunes très foncées; asques

claviformes; spores ellipsoides, dont la longeur n'est

jamais plus de trois fois supérieure à la largeur. . . . . . 63.

63. Espèce corticole; spores $12-17(-21) \times 4,5-6,5 \mu \mathrm{m}$ (fig.

$72 \mathrm{k})$; hypothécium dont la teinte varie entre roux et brun

violacé et dont la coloration pourpre s'intensifie à

l'application de $\mathrm{KOH}$; rebord propre verdâtre, devenant

souvent violet au contact de кон. Espèce peu

fréquente.............. . . . . Catillaria laureri

63. Espèces saxicoles; spores $18-24 \times 8-10 \mu \mathrm{m}$; coloration de

l'hypothécium et du rebord propre brunâtre noir,

$\mathrm{KOH}-$. . . . . . . . . . . . . Rhizocarpon hochstetteri

64. Fructifications couvertes d'un "givre" gris ou d'un

gris nuancé de bleu (pruineuses), noires sous la pruine;

thalle entièrement recouvert de sorédies granuleuses,

vert jaunâtre pâle ou vert clair; spores

quadricellulaires, quelque peu resserrées à la hauteur

des cloisons transversales. Espèce fréquente; corticole

. . . . . . . . . . . . . . . Arthonia caesia

64. Fructifications non pruineuses . . . . . . . . . 65 .

65. Thalle mince ou à peu près invisible (à l'intérieur de

l'(eorce), se manifestant de temps en temps par une "tache"

grisâtre sur l'écorce . . . . . . . . . . . . . . . Arthonia

65. Thalle épais, de granuleux ou pulvérulent, verdâtre ou vert

très foncé. Lichens habitant le bois pourri ou l'écorce. . . . Micarea 


\section{Les espèces CRustacées et squamuleuses (Échantillons stériles)}

1. Espèces terricoles, lignicoles ou saxicoles, parfois muscicoles . . . . . . . . . . . • . . . . . . . . . . . 2 .

1. Espèces corticoles . . . . . . . . . . . . . . . . . 25.

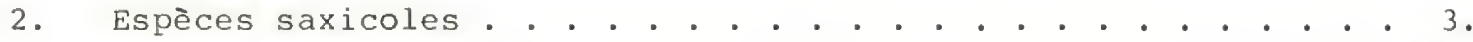

2. Espèces terricoles ou lignicoles, parfois muscicoles . . . . 12 .

3. Thalle noir, renfermant des algues bleues, croissant sur des roches calcaires . . . . . . . . . . . . . . . . . . . . 4.

3. Thalle dont la couleur est variable, non noir, contenant des algues vertes; lichens vivant sur des roches calcaires ou non

4. Thalle orbiculaire, bordé d'un liséré net, bleu ou vert bleuâtre (hypothalle); thalle formé de granulations semblables à des isidies qui s'amassent en plaques aréolées. Espèce commune . . . . . . . . Placynthium nigrum

4. Thalle entièrement pulvérulent, verruqueux ou granuleux; Gloeocapsa est l'algue, encore appelée phycosymbionte. Espèce rare. . . . . . Psorotichia schaereri

5. Thalle entièrement sorédiée (granuleux-pulvêrulent) . . • • • . . . 6.

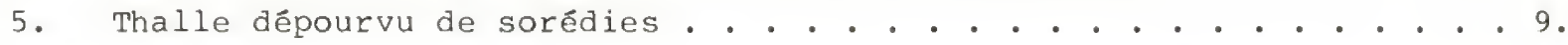

6. Thalle gris bleuâtre ou gris acier; lichens croissant à même le roc dans les habitats ensoleillés . . . . . . • . . . . 7 .

6. Thalle vert ou vert jaunâtre; lichens poussant sur le sol ou sur la tourbe recouvrant les roches, dans des stations ombragées . . . . . . . . . . . . . . . . . . 8 .

7. Thalle Pd+ jaune foncé, KoH+ jaune (acide alectorialique), formant fréquemment des plaques orbiculaires et zonées; très grosses granules. Espèce souvent rencontrée dans les stations ensoleillées . . . . . . . . . . . . . . Lepraria zonata

(N.B.: On peut appeler L. Zobificans Nyl. les membres d'une population contenant de l'atranorine et de l'acide fumar-protocétratique ou protocétratique (Pd+ rouge).) 
7. Thalle $\mathrm{Pd}-$, $\mathrm{KOH}+$ jaune (etranorine), mince, gris bleuâtre, mal dêlimité, non zoné. Espèce commune sur les rochers ombragés et au pied des arbres... . . . . . . . Lepraria incana

8. Thalle constitué d'une épaisse croûte mal délimitée, KOH+ jaune, Pd+ orange (acide stictique et zéorine). Espèce commune sur les pierres et au pied des arbres

8. Thalle composé de petites excroissances de sorédies, semblables à des écailles et membraneuses, koH+ jaune, Pd+ orange (acides pannarique et roccellique, et atranorine). Espèce rare; s'installe sur les parois rocheuses à l'ombre . . . . . . . . . Lepraria membranacea (N.B.: espèce qui ne se trouve peut-être pas dans la région d'ottawa.)

9. Thalle roux, épais, squamuleux, bord des squamules blanc; médulle $\mathrm{KOH-}, \mathrm{C-}, \mathrm{Pd}-$. Rare; s'installe sur le calcaire

9. Couleur du thalle qui varie entre vert grisâtre et vert Psora russellii jaunâtre, surface uniforme, aréolée, ou entre les deux, ou encore thalle lobé...................... . 10.

10. Thalle vert grisâtre, KoH+ jaune, Pdt orange (acide stictique), mal délimité, surface aréolée ou uniforme, ou encore entre les deux. Taxon rare; lichen d'habitats ombragés . . . . . . . . . (fig. 60) Baeomyces rufus

10. Thalle vert jaunâtre (acide usnique), orbiculaire, lobé au pourtour. Espèces d'habitats ensoleillés. . . . . . . 11.

11. Lobes thallins épais, ne devenant pas aréolês au centre, médulle $\mathrm{KOH}-, \mathrm{Pd}-$ et $\mathrm{C}-$. Espèce commune; vit sur les roches calcaires ou rarement au sommet de roches non calcaires où viennent se poser les oiseaux... . . . . . . . Lecanora muralis

11. Lobes thallins minces, fortement appliqués contre le substratum, devenant aréolés sauf au pourtour; médulle Ct rouge ou parfois Pd rouge. Espèce peu fréquente; crô̂t sur les roches non calcaires...... (fig. 22) Dimelaena oreina

12. Thalle jaune orangé, $\mathrm{KOH}+$ pourpre, aréolé, dont les aréoles deviennent sorédiées. Espèce peu fréquente; 
s'installe sur les clôtures de bois et sur l'écorce .............. . . . . Caloplaca microphyllina

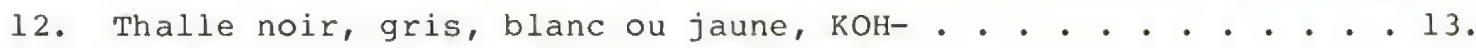

13. Thalle squamuleux ................. . . 14 .

(N.B.: voir également la section consacrée aux Cladonia

dans la clef des lichens fruticuleux.)

13. Thalle aréolé, verruqueux ou sorédié et mal délimité . . . . . 20.

14. Espèces qui croissent sur le sol et sur le calcaire . . . . . 15.

14. Espèces qui croissent sur le bois et les tronçons

d'arbres moussus . . . . . . . . . . . . . . . 18 .

15. Thalle composé d'épaisses squamules rousses à la marge

blanche, ni sorédiées, ni imbriquées; médulle KoH-, C-, Pd-.

Rare; s'installe sur les sols calcaires et le calcaire

. . . . . . . . . . . . . . . . . . psora russellii

15. Thalle composé de squamules minces, imbriquées d'habitude,

vert-de-gris sur le dessus et blanches en-dessous; $\mathrm{KOH}+$

jaune sur la face inférieure (atranorine). Espèces

terricoles . . . . . . . . . . . . . . . . . 16 .

(N.B.: Bien des espèces de Cladonia dépourvues de

podétion peuvent être identifiées ici. Les trois espèces

qui suivent sont les espèces dépourvues de podétions, les

plus fréquemment récoltées.)

16. Squamules Pd+ jaune vif (acide psoromique), grandes,

Etroites ( 4 - $6 \mathrm{~mm}$ de long, 2 - $3 \mathrm{~mm}$ de large),

ascendantes et retroussées, révélant ainsi leur face

inférieure blanche. Rare...... . . Cladonia dahliana

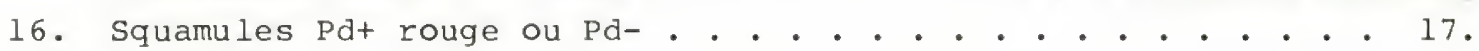

17. Très grandes squamules ascendantes, dont la largeur dépasse souvent $6 \mathrm{~mm}$, profondêment lobées et irrégulières; Pd+ rouge (acide fumar-protocétrarique). Peu fréquent... . Cladonia turgida

17. Petites squamules, dont la longeur n'excède pas $3 \mathrm{~mm}$, entières ou légèrement lobées; régulières; Pd+ rouge ou $\mathrm{Pd}$-.

Commun . . . . . . . . . . . . . . Cladonia cariosa

18. Squamules du thalle sorédiées, non lobées. S'installe sur le bois quelque peu carbonisé . . . . . . . . . . . 19. 
18. Squamules du thalle finement lobées, allongêes, ramifiées, non sorédiées; Pd+ rouge (acide

fumar-protocétrarique). Rare; vit sur les tronçons d'arbres moussus... . . . . . . . Cladonia caespiticia

19. Squamules semblables à des écailles, bombées, pourvues de sorédies se formant sur le revers; thalle C+ rouge, Pd(acide lécanorique). Espèce fréquente

(fig. 2l) Hypocenomyce scalaris

19. Squamules plates ou légèrement bombées, sorédiées au pourtour; thalle Pdt rouge, C- (substance inconnue). Taxon rare... . . . . . . . . . Hypocenomyce anthracophila

20. Thalle aréolé-uniforme, non sorédié ( $v$. doublet 10) ................ (fig. 60) Baeomyces rufus

20. Thalle sorédié: soralies le couvrant entièrement ou s'étalant en plaques distinctes . . . . . . . . . . . . 21 .

21. Thalle vert jaunâtre vif, granuleux-pulvérulent, K-, Pd-, C-. Espèce rare . . . . . . . . . . . . . . . Coniocybe furfuracea

21. Thalle vert ou gris, C+ rose ou rouge... . . . . . . . . . 22 .

(N.B.: Le thalle de Lecidea botryosa est gris cendré foncé, verruqueux ou grossièrement granuleux et devient sorédié par endroit tout comme $L$ granulosa. Néanmoins, il ne réagit pas au contact de $C$ (il renferme de l'acide perlatolique plutôt que de l'acide gyrophorique). Il a été repéré à l'ouest de l'Outaouais et il est probable qu'il vit également ici.)

22. Soralies s'étalant en plaques distinctes sur un thalle continu ou verruqueux, qui est teinté de vert-de-gris pâle ou de gris... . . . . . . . . . . . . . . 23.

22. Thalle lépreux, pulvérulent-granuleux, couvert de sorédies granuleuses, de couleur jaune verdâtre. Espèce rare; lignicole. . . . . . . . . . Micarea viridescens

23. Thalle grossiērement verruqueux, dont quelques verrues se désagrègent et forment des soralies granuleuses de couleur blanchâtre ou rosâtre. Espèce ordinairement terricole, rarement lignicole... . . . . . . . . . Lecidea granulosa

23. Thalle continu, granuleux, pourvu de soralies jaunâtres... . . 24 . 
24. Petites soralies, taille des sorédies variable: fines à granuleuses; mince thalle présentant en lumière ultraviolette des teintes de fluorescence oranges (grande onde). Espèce fréquemment corticole mais rarement lignicole... . . . . . . Ochrolechia arborea

24. Grandes soralies irrégulières, grossièrement granuleuses; épais thalle rugueux, insensible à UV. Taxon rare; muscicole et lignicole... . Ochrolechia androgyna

25. Teinte du thalle s'échelonnant entre le couleur jaune d'oeuf et orange . . . . . . . . . . . . . . . . . . 26 .

25. Coloration du thalle s'échelonnant entre vert et gris ou blanc..................... . . 29.

26. Thalle totalement recouvert de sorédies granuleuses ou granuleux, $\mathrm{KOH}-$. . . . . . . . . . . . . . . . . 27.

26. Morphologie du thalle variable: aréolée à presque squamuleuse, certains compartiments devenant sorédiés; thalle $\mathrm{KOH}+$ (pourpre). Espèce peu fréquente; pousse sur l'écorce d'orme ou sur le vieux bois . . Caloplaca microphyllina

27. Thalle granuleux-pulvérulent, granuleux, non sorédié; taille des granulations atteignant environ 0,05-0,15 mm. Espèce fréquemment rencontrêe, principalement sur l'orme .............. (fig. 4) Candelariella xanthostigma

27. Thalle granuleux-pulvérulent . . . . . . . . . . . . 28.

28. Soralies maculiformes, dont les petites plaques proviennent de la rupture de minuscules granules sphériques et de celle d'aréoles; absence totale de lobes foliacés. Espèce peu fréquente; s'installe sur différentes sortes d'écorce. . (fig. 3) Candelariella efflorescens

28. Soralies capitiformes (pas maculiformes); thalle pourvu de quelques lobes foliacés, ordinairement évidents. Taxon fréquent, s'installant sur différentes sortes d'écorce....... Candelaria concolor var. effusa

29. Thalle lépreux (entièrement composé de sorédies) . . . . . . . . 30.

29. Thalle pourvu de soralies distinctes (pouvant devenir confluentes sur les parties du thalle plus âgées) . . . . . . 33.

30. Thalle pourvu d'un net hypothalle blanc et fibreux

("fongique"); thalle vert jaunâtre, mince, KOH+ 
jaunâtre, C- et Pd- (atranorine, zéorine, acide

usnique). Espèce commune; pousse surtout sur les

érables à sucre . . . . . . . . . . . Lecanora thysanophora

30. Hypothalle blanc faisant défaut; coloration du thalle

variant entre vert clair et vert bleuâtre, jamais vert

jaunâtre (absence d'acide usnique) . . . . . . . . . 31.

31. Thalle épais, verdâtre pâle, KoH+ jaune, Pd+ orange (acide

stictique et zéorine). Commun . . . . . . . . . Lepraria finkii

31. Thalle mince, gris bleuâtre............... . 32 .

32. Thalle Pd-, KC-, KOH- ou KOH+ jaunâtre (atranorine et zéorine) . . . . . . . . . . . . . . Lepraria incana

32. Thalle Pd+ jaune vif, KC+ rouge, $\mathrm{K} \pm$ (acide alectorialique). Peu fréquent

. . . . . . . Lepraria sp. (no 2 au sens de Harris, 1977)

33. Thalle et sorédies C+ rouge (acide gyrophorique); thalle gris pâle, lisse (ou qui a la texture de son substrat:

l'écorce); soralies en petits amas, souvent jaunâtres, UV+

orange (lichexanthone). Rare......... Ochrolechia arborea

33. Thalle et sorédies C- . . . . . . . . . . . . . . 34.

34. Soralies Pd-, $\mathrm{KOH}-$. . . . . . . . . . . . . . 35 .

34. Soralies $\mathrm{Pd}+$ jaune ou orange, $\mathrm{KOH}+$ jaune ou rouge ou soralies Pdt rouge, KOH- . . . . . . . . . . . . . 36 .

35. Soralies blanches (ce sont en fait des ascocarpes qui peuvent ou non renfermer des asques fertiles); thalle mince. Peu

fréquent; vit sur différents types d'écorce. . Pertusaria ophthalmiza

35. Soralies vert jaunâtre, grossièrement granuleuses. Rare;

s'installe au pied des arbres, des frênes surtout

......... . Lecidea sp. (no 4 au sens de Harris, 1977)

36. Soralies Pd+ rouge (acide fumar-protocétrarique),

blanchâtres, granuleuses. Rare.. . . . Lecidea cinnabarina

(N.B.: Pertusaria multipunctoides, qui est pourvu

de verrues fructifères sorédiées, tourne également au

rouge après l'application de Pd.)

36. Soralies Pdt jaune ou orange, ou Pd- . . . . . . . 37. 
37. Thalle $\mathrm{KOH}+$ jaune vif, Pd+ jaune orangé (acide thamnolique); couleur du thalle s'echelonnant entre gris pâle et blanc, pustules thallines creuses qui, en éclatant, deviennent des sorêdies granuleuses. Peu fréquent... . . . . . Haematomma sp. (N.B.: Pertusaria trachythallina, pourvu de verrues fructifères sorédiées et fertiles, renferme également de l'acide thamnolique.)

37. Thalle $\mathrm{KOH}+$ jaune ou rouge, Pdt entre jaune pâle et jaune foncé ou Pd- (pas d'acide thamnolique); les sorédies ne proviennent pas de l'éclatement de pustules creuses . . . . . . 38 .

38. Thalle KOH+ jaune, Pd- ou Pd+ jaune pâle (atranorine); thalle gris pâle, parsemé de groupes de sorédies distincts (souvent jaunâtres). Commun; pousse sur les arbres en bordure des routes . . . . . . . Lecanora impudens

38. Thalle $\mathrm{KOH}+$ jaune virant au rouge, Pd+ jaune (acide norstictique); thalle gris pâle, verruculeux, qui, en se désagrégeant par endroits, se transforme en sorédies granuleuses. Rare; corticole......... Phlyctis argena 


\section{ACAROSPORA}

1. Thalle ou disque des apothécies couvert d'une couche de pruine, mince ou épaisse; coloration des arẻoles variant entre vert-de-gris et brun grisâtre; compartiments ayant $4 \mathrm{~mm}$ de large et lobés, ou, dans de rares cas, se réduisant à la marge de l'apothécie; apothécies d'abord enfoncées dans le thalle et devenant lécanorines par la suite. Espẽce Eréquente sur les roches calcaires. . . . . . . . . . A. glaucocarpa

1. Thalle et apothécies dépourvues de pruine; aréoles brunes,

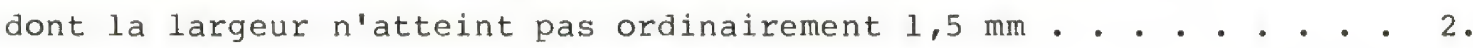

2. Une fois à maturité, apothécies devenant souvent sessiles, pourvues d'une marge distincte, noire, presque du type lécidéin; arẻoles luisantes. Espèce rare; colonise les roches non calcaires . . . . . . A. badiofusca

2. Apothécies enfoncées dans le thalle ou devenant lécanorines (de même couleur que le thalle), une fois à maturité . . . . . . . . . . . . . . . . . . . 3 .

3. Aréoles brun luisant; chaque aréole contient ordinairement un petit nombre d'apothécies qui demeurent enfouies dans le thalle et qui s'ouvrent par de petites ouvertures; thalle C+ rouge (difficile à observer). Espèce commune; colonise le granite et d'autres roches non calcaires dans les milieux ouverts... . . . . . . . . . . . . . (fig. 1) A. fuscata

3. Aréoles d'un brun rougeâtre terne; une seule apothécie par compartiment, chacune d'elles étant lécanorine à maturité. Taxon rare; calcicole. . . . . . . . . . . . . . . A. canadensis

\section{ARTHONIA}

1. Ascocarpes gris bleu, pruineux, ronds; couleur du thalle s'échelonnant entre vert jaunâtre et vert grisâtre, ce dernier étant lépreux spores quadricellulaires. Espẽce commune; vit sur les arbres. . . . . . . . . . . . . . . A. caesia

1. Ascocarpes non pruineux, dont la coloration s'échelonne entre noir et brun foncé; morphologie du thalle variable; lisse à peu visible. . . . . . . . . . . . . . . . . . . . . . . 2 . 
2. Ascocarpes élancés, souvent ramifiés . . . . . . . . . . . 3 .

2. Ascocarpes ronds ou quelque peu irréguliers, ni élancés,

ni ramifiés . . . . . . . . . . . . . . . 5.

3. Spores bicellulaires; thalle hypophléode, laissant sur l'ecorce une "tache" ou plaque blanche ou gris clair; ascocarpes minces, délicats (souvent n'atteingnant pas 0,1 mm de largeur), à une seule ramification souvent. Espèce commune; vit sur l'écorce d'arbres à feuilles caduques, surtout l'érable . . . . . . . . . . . . . . . A. dispersa

3. Spores quadricellulaires, thalle gris pâle ou à peine visible; ascocarpes plus larges, souvent ramifiés, de 0,15 à $0,35 \mathrm{~mm}$ de large. Taxons s'installant sur divers types d'écorce . . . . . . . . . . . . . . . . . . 4 4

4. Ascocarpes demeurant noirs au contact de l'eau; partie supérieure de l'hyménium bistre. Lichen peu fréquent

4. Ascocarpes devenant brun translucide au contact de (fig. 10) A. radiata l'eau; partie supérieure de l'hyménium brun clair. Lichen fréquent..... A. sp. (no 3 au sens de Harris, 1977)

5. Spores bicellulaires; ascocarpes noirs, ronds, semblables à ceux des Lecidea; thalle laissant paraître une plaque claire sur l'écorce. Lichen rare; habite sur l'écorce de peuplier. . . . . . . . . . . . . . . . A. patellulata

5. Spores contenant 4 ou 5 cellules (mais ordinairement absentes); ascocarpes de forme irrégulière; thalle pratiquement absent. Lichen peu fréquent; s'installe sur les arbres à feuilles caduques . . . . . . . . . . A. punctiformis

ARTHOPYRENIA S.1.

1. Spores à une cloison, dont la cellule inferieure est un peu plus longue que la cellule superieur (dans l'asque); pseudo-paraphyses consistant en cellules courtes, epaisses et très ramifiês. Rare; pousse habituellement sur l'écorce lisse, p. ex., celle de l'erable rouge ou de l'aulne 
1. Spores à $l(-3)$ cloisons, dont la cellule inférieure est nettement plus courte que la ou les cellules supérieures (dans l'asque); pseudo-paraphyses élancées, filiformes, ramifiés. Rare; s'installe sur l'écorce tendre ou épaisse, p. ex. celle de l'orme, de l'érable à sucre et du frêne . . . . . Anisomeridium willeyana (syn. Arthopyrenia willeyana)

\section{ARTHOTHELIUM}

1. Thalle blanc, ordinairement bien développé; ascocarpes $\mathrm{KOH}+$ rouge violacé, du moins en partie; spores 30-35 x 15-20 um, hyalines, murales ou submurales. Taxon peu fréquent;

s'installe sur les arbres à feuilles caduques, surtout l'erable . . . . . . . . . . . . . . . . . A. spectabile

1. Thalle mince ou peu visible, ne laissant voir en général qu'une tache grise; ascocarpes KOH-; spores $16-25 \times 5-10 \mu \mathrm{m} . . .2 .2$.

2. Spores devenant brun foncê, larges $(9-10 \mu \mathrm{m})$; tissu fertile (c.-à-d. la couche ou se trouvent les asques) verdâtre. Espèce rare; corticole... . . . . . A. ruanideum 2. Spores hyalines, etroites $(5-7,5 \mu \mathrm{m})$; tissu fertile noir brunâtre. Espẽce rare; corticole........ A. anastomosans

\section{ASPICILIA}

1. Thalle orange pâle; disque des apothécies orange rosâtre; spores 11-17 × 5-6,5 $\mu \mathrm{m}$; espèce fréquente, colonisant les rochers qui sont couverts au moins periodiquement par l'eau

1. Thalle allant de vert-de-gris à gris cendré; lichens non aquatiques . . . . . . . . . . . . . . . . . 2 .

2. Disque des apothécies brun rougeâtre; thalle vert-de-gris foncé, KOH-; longeur des spores n'atteignant pas 10 um. Espèce rare . . . . . . Lecanora sp.

2. Disque des apothécies noir; thalle gris cendrê pâle ou

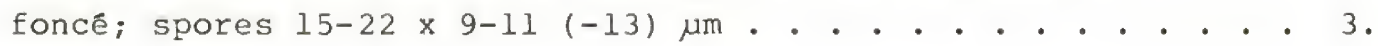


3. Thalle (cortex et medulle) KоH-, Pd-; thalle assez epais, gris cendré, fendillé-arêlé; apothécies plus ou moins en forme de cratère, dont le disque est souvent bordé de blanc; spores en forme d'ellipse large, 15-18 x 11-12 $\mu \mathrm{m}$ ............... . . cf. verrucigera (lignée inactive)

3. Thalle (le cortex ou la médulle ou les deux) KoH+ jaune ou rouge; thalle gris cendré foncé habituellement, pâle parfois; ordinairement verruqueux-arélé . . . . . . . . . . . . . . 4 .

4. Cortex ou médulle du thalle $\mathrm{koH}+$ rouge (acide norstictique); thalle uniforme, fendillé-aréolé ou un peu verruqueux (fig. 2). Fréquent . . . . . . . A. cinerea 4. Cortex ou medulle du thalle koH+ jaune persistant (acide stictique) . . . . . . . . . . . . . . 5 .

5. Thalle areole ou abondamment verruqueux, les verrues devenant un peu lobées ou presque squamuleuses dans les parties âgées. Frequent . . . . . . . . . . . . . . A. verrucigera

5. Thalle lisse, mince, continu ou fendille et finalement aréolé, gris cendré pâle. peu fréquent... A. cinerea var. laevata

BACIDIA

1. Lichens saxicoles; spores 25-35 x 2-3 $\mu$, aciculaires . . . . . . 2 .

1. Lichens muscicoles, lignicoles ou corticoles . . . . . . . . . . . 3 .

2. Spores incurvêes et spiralées dans l'asque; hypothécium hyalin, epithecium brun. Espèce rare.. Scoliciosporum umbrinum

2. Spores droites, bacillaires; hypothécium brun rouille sur le dessus, hyalin en dessous, ou jaune pâle; épithécium érugineux; rebord propre rouge violacé, coloration qui s'intensifie par $\mathrm{KOH}$. Espèce rare

(N.B.: Il s'agit peut-être de l'espèce no $l$ au sens de R. Harris 1977 , le vrai $B$. inundata possēde un épithécium brunâtre.)

3. Lichens muscicoles . . . . . . . . . . . . . . 4 .

3. Lichens corticoles et lignicoles . . . . . . . . . . . . 7 .

4. Spores fusiformes . . . . . . . . . . . . 5 . 
4. Spores aciculaires, 3 - 7 septums, $35-40 \times 2,5-3,5 \mu m$; épithêcium verdâtre; hypothêcium brun, mince; rebord propre surtout incolore à pourpre pâle au contact de $\mathrm{KOH}$. Rare . . . . . . . . . . . . . . . B. bagliettoana

5. Apothécies s'échelonnant entre jaunâtre pâle et chamois, ces dernières ne fonçant jamais (exception faite des spécimens d'herbier mal séchés), étant très bombées une fois à maturité; hypothécium jaune pâle; spores invariablement à trois cloisons, $15-20 \times 5-6 \mu m$; thalle granuleux. Espèce rare . . . . . . . . . . . . . . . . . . . . B. sphaeroides

5. Apothécies variant entre brun clair et noir,

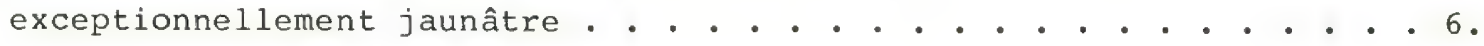

6. Spores à 3-5 cloisons, 18-30 x 5,7-7,5 $\mu \mathrm{m}$; hypothêcium brun rougeâtre, brun ou exceptionnellement brun clair (surtout sur le dessus); hypothécium KOH+ violet rougeâtre; thalle grossièrement granuleux ou presque squamuleux. Espèce fréquente . . . . (fig. 13) B. sabuletorum

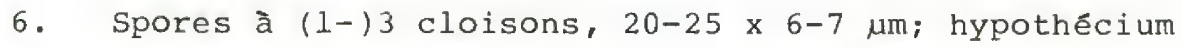
hyalin à brun clair; apothécies variant entre brun jaunâtre et brun rougeâtre ou noir, leur marge étant concolore au disque ou plus pâle que celui-ci; thalle granuleux. Espèce peu fréquente . . . . . B. obscurata

7. Minuscules apothécies, dont le diamètre s'échelonne entre 0,10 et $0,16 \mathrm{~mm}$, noires, hémisphériques et dépourvues de bord distinct; thalle vert foncé, granuleux à verruqueux . . . . . . . . . 8 .

7. Largeur đes apothécies: $0,25-1,6 \mathrm{~mm}$; coloration thalline qui s'échelonne entre vert-de-gris et vert olive; spores aciculaires . • • • • • • • • • • • • • • • • • • • • • • 9 .

8. Spores fusiformes ou presque aciculaires, ordinairement fortement effilées à l'une de leurs extrémités, 25-33 x

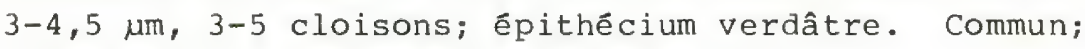
vit à l'ombre sur l'écorce et le bois... . . . . . . . . . . . . . (fig. 12) scoliosporum chlorococcum

8. Spores fusiformes, $16-19 \times 5-6 \mu \mathrm{m}, 1$ à 3 cloisons. Peu fréquent; vit sur les tronçons d'arbre et le bois en décomposition . . . . . . . . . . . . . Micarea melaena 
(11)
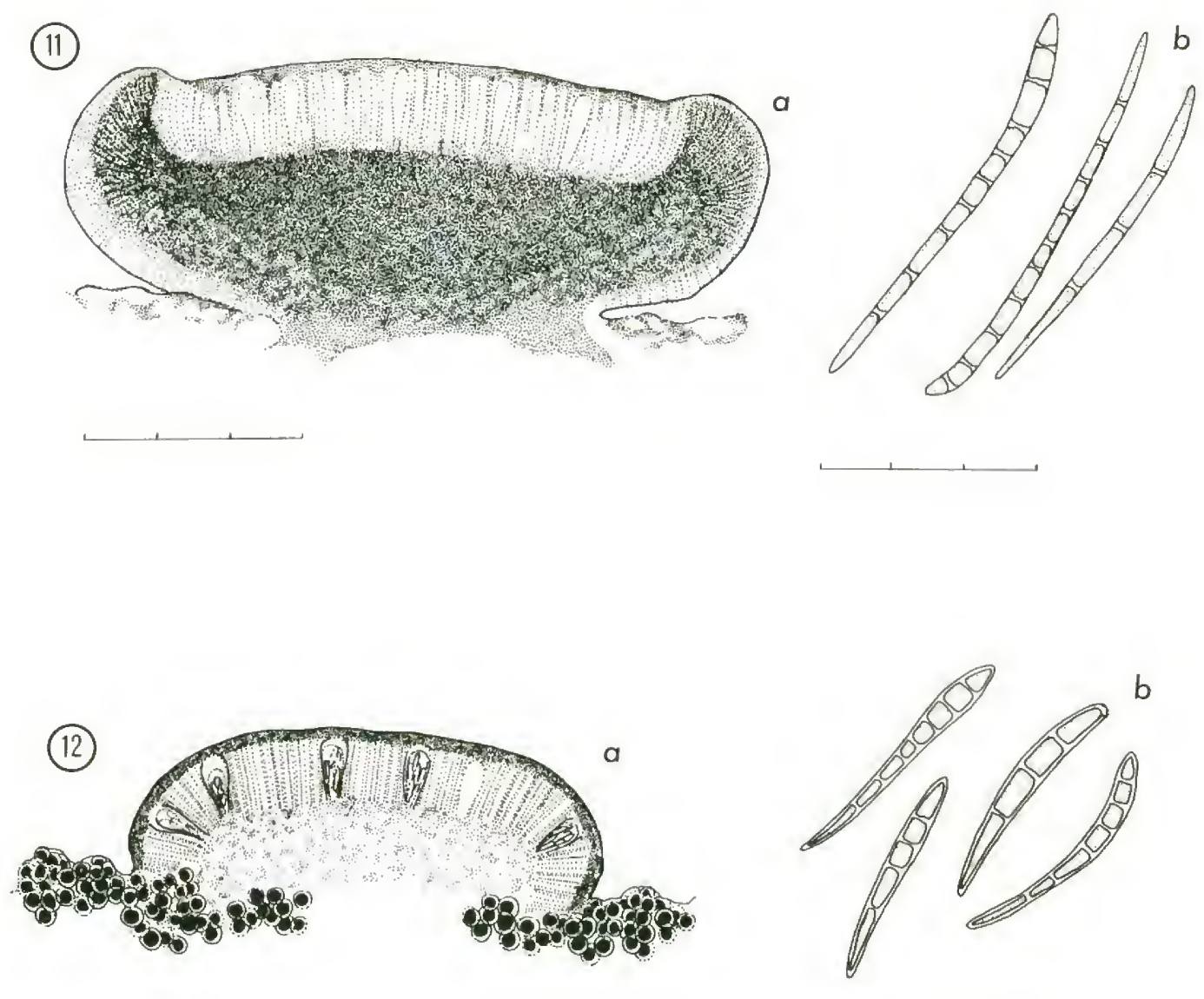

b

(13)

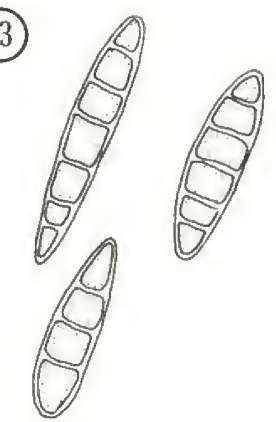

Figures 11 à 13.-11. Bacidia schweinitzii: a) coupe verticale d'une apothécie; b) ascospores.-12. Scoliciosporum chlorococcum: a) coupe verticale d'une apothécie; b) ascospores.-13. Bacidia sabuletorum: ascospores. Échelle: -11a, 12a: chaque division $=100 \mu \mathrm{m} ;-11 \mathrm{~b}, 12 \mathrm{~b}, 13$ : chaque division $=10 \mu \mathrm{m}$. 
9. Spores à (1-)3 cloisons, 25-31 x 1,3-2,5 um; diamètre des apothécies: 0,25-0,35 mm; leur coloration variant de noir à noir brunâtre; fructifications à marge mince; hypothécium brun jaunâtre pâle; êpithécium vert; couleur de l'intérieur du rebord propre s'échelonnant entre la transparence et le rose, bord du même tissu étant noir verdâtre. Espèce rare; vit sur le bois (et l'écorce) . . . . . . . . . . . B. beckhausii

9. Spores à 3-15 cloisons, de plus de 30 um de long; diamètre des apothécies: $0,4-1,6 \mathrm{~mm}$. . . . . . . . . . . . . . . . 10 .

10. Grandes apothécies de 0,8-1,6 mm de diamètre, nettement resserrées à la base; disque aplani, noir ébène, au bord mince mais proéminent; hypothécium brun, se fondant dans le rebord propre roux, dont la couleur varie entre une teinte plus claire de roux et la transparence; épithécium vert; spores à 3-8 cloisons, 30-55 x 2-3 $\mu$; thalle lisse à granuleux ou presque isidié. Espèce fréquente; corticole, se rencontre dans les forêts fermées............. (... (fig. 11) B. schweinitzii

10. Apothêcies de $0,4-0,8 \mathrm{~mm}$ de diamètre, fortement appliquées contre le thalle; si elles sont noires, leur bord ne surplombe pas le disque (non proéminent) et disparaît à maturité . . . . . . . . . . . . . . . . 11 .

11. Apothécies noires, jamais pruineuses; bord évanescent; hypothécium brun rougeâtre; épithêcium vert olive grisâtre; face interne du rebord propre incolore à brun jaunâtre;

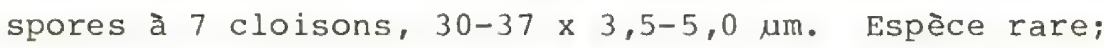

corticole. . . . . . . . B. accedens (au sens de Harris, 1977)

11. Coloration des apothécies variant entre une teinte sombre et une nuance claire de brun rougeâtre ou de brun jaunâtre;

fructifications fréquemment pruineuses, du moins

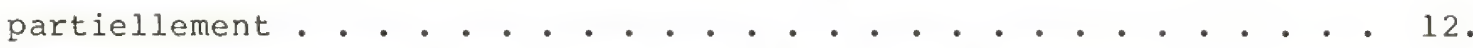

(N.B.: B. atrogrisea (Del. ex Hepp) Körb. est une espèce voisine, qui n'a pas encore été signalée dans l'Outaouais, mais qui s'y trouve probablement. La couleur des apothécies varie entre brun-rouge foncé et noir et elles sont dépourvues de pruine; hypothécium hyalin à jaunâtre; épithécium parsemé de pigments pourpre sombre; spores qui ont jusqu'à 18 cloisons, $45-60(-70) \times 2,2-4,0 \mu \mathrm{m}$.) 
12. Thalle composé de squamules qui s'allongent et ressemblent à des isidies; les spores contiennent

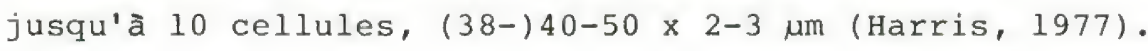
Rare . . . . . . . . . . . . . . . B. rubella

12. Thalle non isidié-granuleux . . . . . . . . . . 13.

13. Coloration des apothécies s'échelonnant entre brun rougeâtre vif et brun jaunâtre, devenant rarement brun rougeâtre très foncé (presque noir); certaines apothécies sont souvent légèrement pruineuses, surtout au pourtour; bord bas, qui a fréquemment tendance à disparaître; hypothécium brunâtre, au moins dans la partie supérieure; couleur du rebord propre variant entre la transparence et le brun franc ou le brun pourpre; rebord propre et hypothécium KOH+ violet rosâtre; thalle lisse; spores à 3-7 cloisons, (30-)33-35 x 2-3(-4) $\mu \mathrm{m}$. Lichen fréquent; habite l'écorce d'arbres feuillus . . B. fuscorubella

13. Apothécies brun rougeâtre foncé, à bord proéminent, habituellement très pruineuses sur le disque et le bord; coloration de l'hypothécium allant de la transparence au jaune claire, rebord propre KOH+ jaune pâle (jamais violet rosâtre); thalle devenant épais, fendillé; spores à 6-12(-15) cloisons, 45-72 × 3-4 $\mu$ m. Espèce peu fréquente; pousse sur les essences décidues . . . . . . . . . . . . B. suffusa

\section{BUELLIA}

1. Thalle colonisant les roches non calcaires, dont la couleur varie de gris cendré à gris brunâtre, épais, verruqueux; apothécies légèrement convexes, dont le bord est mince ou peu visible; spores 9,5-13,0 x 5,5-7,0 $\mu \mathrm{m}$. Taxon rare. . . B. turgescens

1. Taxons corticoles ou lignicoles . . . . . . . . . . . . . . . . 2 .

2. Grandes apothécies de 0,5-1,2 mm de diamètre, planes, pourvues d'un bord proéminent et persistant; spores 12-17 × 5-7 um; des coupes provenant des apothécies $\mathrm{KOH}+$ jaune, des cristaux rouges finissent par apparaitre dans la plupart des cas (acide norstictique). Taxon commun; s'installe sur l'écorce de feuillus et de conifères 
2. Petites apothécies dont le diamètre n'atteint pas 0,5 mm, en règle générale; absence d'acide norstictique . . . . . . 3 .

3. Spores au nombre de 12-24 par asque, 8,8-9,5 x 4,8-4,9 $\mu$;

intérieur du rebord propre pâle, extérieur noir; bord de l'apothécie mince et persistant. Lichen rare, rencontré sur les essences feuillues . . . . . . . . . . . . . . . B. polyspora

3. Spores au nombre de 8 par asque, $11-20$ x $5-8 \mu m$. . . . . . . . . 4.

4. Thalle assez épais dont la morphologie va de verruculeuse à granuleuse, vert-de-gris, Pd+ rouge (acide fumar-protocétrarique); apothécies globuleuses, devenant immarginēes; spores $14-20 \times 7,5-8,0 \mu \mathrm{m}$. Taxon rare; s'installe sur les conifères, en particulier Tsuga . . . . . . . . . . . . . . . . B. dialyta

4. Thalle mince et blanc, ou à peine visible, insensible à Pd; apothécies normalement aplaties, entourées đ'un bord persistant et mince; spores $12-15 \times 6-7 \mu \mathrm{m}$. Espèce peu fréquente; corticole et lignicole, surtout rencontrée sur Ulmus . . . . . . . . . . . . . . . B. punctata

\section{CALICIALES}

1. Ascocarpes dépourvues de pied; mazédium dans des verrues thallines, ressemblant à des apothécies lécanorines; thalle jaune verdâtre; spores bicellulaires. Espèce peu fréquente; se trouve sur le vieux bois dur. . . . . (fig. 7) Cyphelium tigillare

1. Ascocarpes portés par un pied filiforme, court ou long . . . . . . 2.

2. Espèce parasitant d'autres lichens (surtout

Lecanora et Pertusaria); pied extrêmement court, gris ou pâle; spores globuleuses, de 8-10 um de diamètre. Taxon rare . . . . . . . Sphinctrina microcephala

2. Lichens non parasites, corticoles, lignicoles ou muscicoles . . . . . . . . . . . . . . . . . . 3 . .

3. Spores cloisonnees, brunes. (fig. $72 j$ ) . . . . . . . . . . . . 4.

3. Spores non cloisonnẻes, brunes ou incolores (fig. $72 i$ ). . . . . 6 .

4. Espèce vivant sur les rameaux âgës de 2-4 ans de Rhus 
typhina (vinaigrier); pied n'atteignant pas $0,4 \mathrm{~mm}$ de haut, simple ou à une seule ramification; ascocarpes totalement noirs; spores brun clair, lisses, ellipsoïdes, à peine resserrées, $11,5-14,5 \times 3,5-5,0 \mu \mathrm{m}$. Taxon fréquent.......... . . Phaeocalicium curtisii

4. Espèces lignicoles; hauteur du pied dépassant $0,5 \mathrm{~mm}$ (jusqu'à l mm) . . . . . . . . . . . . . . . . . 5 .

5. Spores brunes foncées, resserrées aux cloisons, à surface

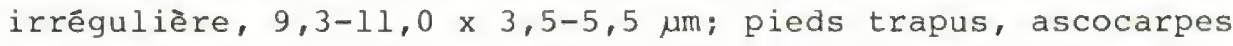
entourés d'une marge jaunâtre et distincte habituellement; pycnides urcéolées, abondantes souvent, dont l'ostiole est bordé d'un lisêré jaunâtre. Fréquent; pousse sur le bois de conifère . . . . . . . . . . (fig. 5) Calicium trabinellum

5. Spores brun pâle, ellipsoïdes à cylindriques, vaguement septées et non resserrées, lisses, 5,5-7,5 x 2,0-2,6 $\mu$ m; pieds élancés, filiformes, rougissant dans l'acide nitrique concentré; ascocarpes dépourvus de marge jaunâtre; pas de pycnides. Rare; pousse sur le bois des essences à feuilles caduques . . . . . . . . . . . . . Chaenothecopsis debilis (N.B.: Espèce signalée par Tibell, 1975; pas de spécimen au CANL.)

6. Spores ellipsoïdes à fusiformes (fig. 72i), brunes, 6-9 x $2,5-3,5 \mu \mathrm{m}$; thalle entièrement absent ou laissant paraître une tache blanche; ascocarpes noirs, plats et presque discoïdes. Taxon fréquemment rencontré sur le vieux bois, en particulier celui des conifères ................ (fig. 6) Mycocalicium subtile 6. Spores globuleuses, de 2,5-4,0 um de diamètre; ascocarpes brunâtres ou jaunâtres . . . . . . . . . . . . . . 7 .

7. Pied des ascocarpes et ascocarpes eux-mêmes recouverts d'une pruine jaune; thalle jaune verdâtre vif, lepreux, assez épais. Lichen poussant souvent au milieu des racines particulièrement ombragés de chablis ou dans d'autres habitats ombragés du même genre. Espèce peu fréquente

7. Pieds noirs, ascocarpes brun sale, dépourvus de pruine jaune Lichen vivant sur le bois mort exposé . . . . . . . . . . . . 8 . 
8. Contour du phycosymbionte carré ou rectangulaire

(Stichococcus) celle-ci étant très petite; thalle voyant, vert sale, lépreux. Lichen rare... . . . . Chaenotheca stemonea

8. Contour du phycosymbionte circulaire (Chlorococcales); thalle absent ou mince, gris bleuâtre, lépreux. Lichen rare ..................... . . . Chaenotheca brunneola

\section{CALOPLACA}

1. Parasite de lichens foliacés (Physciopsis)....... Caloplaca sp. Thalle saxicole, corticole ou lignicole. . . . . . . . . . . . 2 .

2. Thalle saxicole. . . . . . . . . . . . . . . . . . . . . 3 .

2. Thalle corticole ou lignicole. . . . . . . . . . . . . 6 .

3. Thalle fait surtout ou totalement de sorédies granuleuses jaunes, fréquemment stérile. Espèce rare, s'établissant sur le calcaire. . . . . . . . . . . . . . . . . . . c. citrina

3. Thalle dépourvu de sorédies. . . . . . . . . . . . . . . 4 .

4. Thalle bien visible, jaune soufre pâle; apothécies de 0,4-1,2 mm de diamètre, orange clair, dont le bord est presque concolore quand elles sont parvenues à maturité; spores 13-14 x 6-7,5 $\mu \mathrm{m}$; isthme de la spore large, mesurant plus du tiers de la longueur de la spore. Espèce fréquemment rencontrée sur les roches calcaires et le ciment............... . . . f flavovirescens

4. Thalle peu visible ou absent, noirâtre ou gris si présent; isthme de la spore très étroit, moins d'un quart de la longueur de la spore; apothécies orange brunâtre, entourées d'un bord d'une teinte orangée plus pâle... . . . . . . . . . . . . . . . . . 5.

5. Petites apothécies de moins de $0,25 \mathrm{~mm}$ de large; spores 14-16 x (6-)7-9 um (fig. 14); thalle empruntant la forme d'une croûte noirâtre entourant les apothécies. Lichen fréquemment rencontré sur le calcaire et le béton. . . C. feracissima

5. Apothécies de 0,25-0,50 mm de diamètre; spores 10-14 x 4-5 um; thalle ordinairement absent, jamais noirâtre. Lichen fréquent, saxicole... . . . . . . . . . . . . . C. arenaria 
6. Thalle de jaune à orange, кон+ pourpre . . . . . . . . . . 7 .

6. Thalle blanc, gris ou absent.............. 8 .

7. Thalle orange jaunâtre à orange, aréolé à squamuleux, sorédié (granuleux), ordinairement stérile. Taxon fréquent; s'établit sur les arbres poussant le long des chemins et sur les clôtures de bois . . . . . . . . . . . c. microphyllina

7. Thalle jaune soufre clair, plus ou moins continu; apothécies fréquentes, oranges, à bord plus clair. Taxon peu fréquent; s'installe sur les poteaux de clôture....... . . . flavorubescens

8. Apothécies entourées d'un bord gris, bien visible; diamètre des fructifications dépassant normalement 0,5 $\mathrm{mm}$. . . . . . . . . . . . . . . . . . . . 9.

8. Bord des apothécies jaune ou orange dans la plupart des cas; petites apothécies de $0,3-0,5 \mathrm{~mm}$ de diamètre dont la couleur varie entre orange foncé et orange jaunâtre; spores 10,5-13,0 × 6-8 um (fig. 15). Lichen fréquement rencontré sur les ormes, les peupliers et le bois . . C. holocarpa

(14)
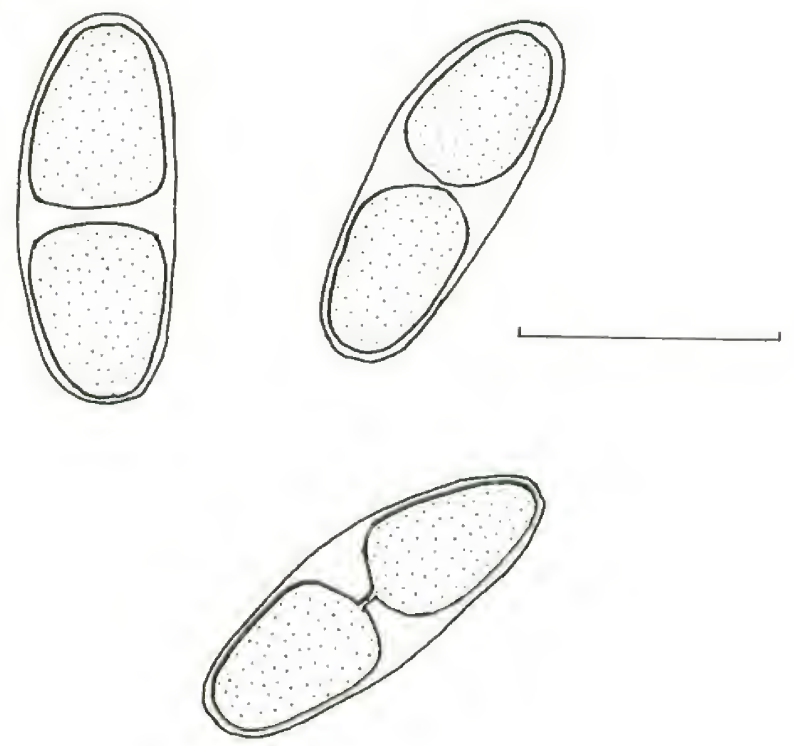

(15)

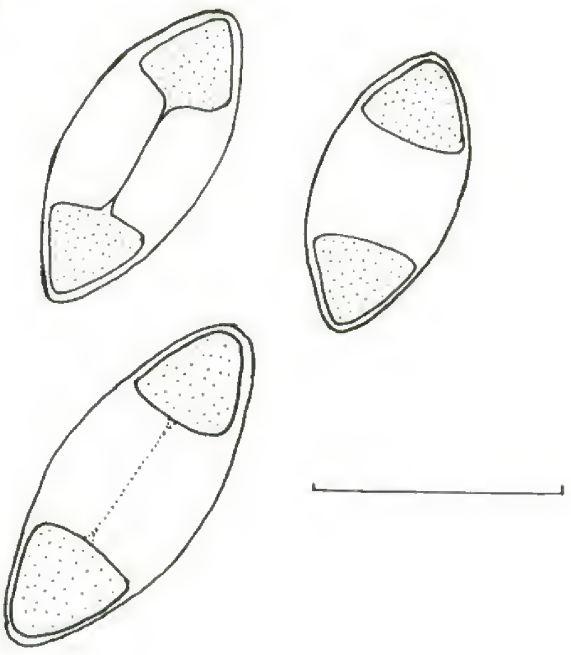

Figures 14 et 15.-14. Caloplaca feracissima: ascospores aux isthmes étroits.-15. Caloplaca holocarpa: ascospores aux isthmes larges. Échelle: chaque division $=10 \mu \mathrm{m}$. 
9. Bord des apothécies couvert d'une pruine blanche; leur disque revêtu d'une pruine jaune; thalle devenant souvent squamuleux; apothécies de 0,5-1,7 mm de diamètre. Lichen peu fréquent; crô̂t sur les arbres situés en bordure des chemins et sur le bois... . . . . . . . . . . . . c. ulmorum

9. Bord et disque des apothécies dépourvus de pruine ou à peu près; thalle gris cendré, non squamuleux; apothécies de 0,4-0,9 mm de diamètre. Lichen fréquent, vit sur les arbres installés le long des chemins et sur les peupliers . . . . C. cerina

\section{CANDELARIELLA}

1. Thalle aréolé, ou couvert de minuscules écailles ou encore absent, ni sorédié, ni finement granuleux; en règle générale, taxons fertiles... . . . . . . . . . . . . . . . 2 .

1. Thalle fait de granulations ou sorédies; taxons ordinairement

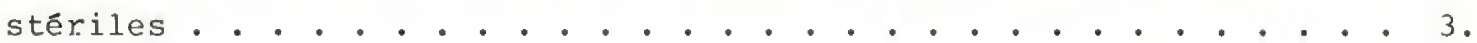

2. Espèce s'établissant sur le calcaire; 8 spores par asque; en règle générale, thalle pas évident. Lichen peu frequent... . . . . . . . . . . . . . . c. aurella

2. Espèce non calcicole ou rarement lignicole; 16-32 spores par asque; thalle ordinairement bien visible compose d'aréoles lobées, grandes ou minuscules. Taxon commun

C. vitellina

3. Thalle consistant en granulations dispersées, nettement cortiqués et rondes. Espèce commune; rencontrée tout particuliērement sur les ormes et Thuja... . (fig. 4) C. xanthostigma 3. Thalle totalement ou presque entièrement lépreux (sorếdié) . . . . 4.

4. Règle générale, thalle composé d'un petit nombre de lobes foliacés entourés de sorédies (ces dernières étant marginales au début); soralies ordinairement diffuses; 32 spores par asque. Taxon fréquent

. . . . . . . . . . Candelaria concolor var effusa

4. Thalle dépourvu de lobes foliacés; aréoles se désintégrent peu à peu, de l'extérieur vers le centre, ce qui forme des soralies maculiformes 32 spores par asque. Taxon peu fréquent...(fig. 3) Candelariella efflorescens 
1. Apothécies de 0,5-2,0 mm de diamètre, dont la coloration s'échelonne entre jaune et orange jaunâtre, aplaties; bord

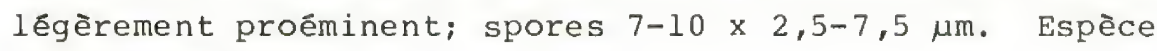
rare; corticole.. . . . . . . . . . . . . . . . . D. lutea

1. Apothécies dont le diamètre atteint $0,5 \mathrm{~mm}$ et dont la couleur varie entre chamois clair et rose terne; spores 10-15 x

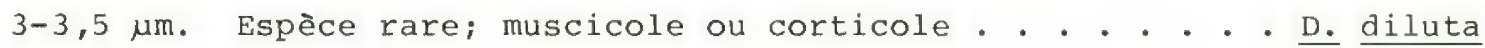

\section{HUILIA}

1. Apothécies couvertes d'une pruine grise, pourvues d'un bord noir, planes; thalle gris cendré, $\mathrm{KOH}+$ jaune foncé à rouge. Lichen fréquent; s'établit sur les roches siliceuses dans les forêts fermées... . . . . . . . . . . H. albocaerulescens

1. Apothécies dépourvues de pruine, souvent fortement convexes; thalle $\mathrm{KOH}-$. . . . . . . . . . . . . . . . . . . . . 2.

2. Très grandes apothécies de 0,5-2 mm de diamètre; spores

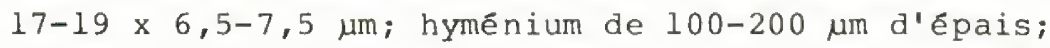
thalle souvent mince ou pratiquement absent. Espèce commune, vivant sur les roches siliceuses exposées . . . . . . . . . . . . . (fig. 20) H. macrocarpa

2. Apothécies de $0,35-0,75 \mathrm{~mm}$ de diamètre; spores $14,5-16,0$ $(-19,5) \times 6,2-7,6(-8,7) \mu \mathrm{m}$; hyménium ayant moins de 100 um d'épaisseur; thalle gris cendré. Espèce commune; poussant sur les rochers exposés. . . . . . . H. crustulata

\section{LECANIA}

1. Spores quelque peu courbées (fig. 72e), 13-16(-18) x 4,5-6,0 $\mu \mathrm{m}$; minuscules apothécies dont le diamètre atteint 0,25 mm et dont la couleur s'échellonne entre brun foncé et noir; bord mince, appelé à disparaître. Espèce peu fréquente; crô̂t sur l'écorce d'arbres à feuilles caduques . . . . . . . . . . L. dimera

1. Spores droites, $(8,5-) 11-13 \times 3,3-4,0 \mu \mathrm{m}$; apothécies de 0,25-0,8 mm de diamètre, roux foncé, convexes; bord mince. 
Espèce fréquemment rencontrée sur les arbres décidus, surtout le frêne noir... . . . . . . . . . . . . L. cyrtella

\author{
LECANORA
}

1. Espèces corticoles ou lignicoles . . . . . . . . . • . . . . . . . 2 .

1. Espèces saxicoles . • . . . . . . . . . . . . . . . . . 23.

2. Thalle ou bord des apothécies sorédié . . . . . . . . . . . . 3.

2. Thalle et bord des apothécies non sorédiés . • • • • • • . • . 5 .

3. Thalle ou bord des apothécies vert jaunâtre (acide usnique) . . . . 4 .

3. Thalle et bord des apothécies gris ou blanc, KOH+ jaune, sorédies granuleuses formant des plaques sur le thalle; apothécies rares, brun rougeâtre vif et bordées d'un lisêré distinct, blanc, souvent sorédié. Lichen fréquent; rencontré surtout sur les arbres le long des chemins . . . . . . . . . L. impudens

4. Thalle stérile, mince, lépreux, bordé, en général, d'un hypothalle blanc et fibreux. Espèce frêquente; s'établit surtout sur l'écorce d'érable à sucre. . L. thysanophora 4. Thalle fertile, pourvu de bon nombre d'apothécies dont la couleur varie entre jaune et brun sale; absence de l'hypothalle blanc et fibreux; thalle non lépreux (en général, il n'y a que le bord des apothécies qui devient sorẻdié). Espèce fréquente; s'installe sur différentes sortes d'écorce et de bois. . . . . . . . . . L . strobilina

5. Disque de toutes les apothécies fortement pruineux . • • . . . . . . 6 .

5. Apothécies dépourvues de pruine à maturité (c.-à-d. que chez un petit nombre d'espèces les jeunes apothécies sont légèrement pruineuses) . . . . . . . . . . . . . . . . . . 8 .

6. Thalle et bord des apothécies KOH-; spores 9-14 x 4-6(-7) $\mu \mathrm{m}$. Taxon rare; corticole ou lignicole... . L. hagenii 6. Thalle et bord des apothêcies koH+ jaune (atranorine) . . . . . 7 .

7. Bord des apothécies persistant et proêminent; grandes apothécies, dont la largeur atteint 1,2 mm; coupes dans I'apothécie (amphithécium) KoHt non pas rouge, mais jaune. Espèce peu fréquente, corticole. . L. caesiorubella ssp. caesiorubella 
7. Bord des apothécies mince ou appelé à disparâtre chez les plus vieilles apothécies; largeur des fructifications plus petite que $0,5 \mathrm{~mm}$, en général; coupes de l'apothêcie $\mathrm{KOH}+$ rouge (acide norstictique). Espèce rare; corticole

. . . . . . . . . . . . . L. pallida var rubescens

8. Bord des apothécies, leur disque ou thalle vert jaunâtre ou jaunâtre... . . . . . . . . . . . . . . . . 9.

8. Coloration du bord des apothécies, de leur disque et du thalle variant entre gris sale et blanc, dépourvue de teinte jaunâtre . . . . . . . . . . . . . . . . 12 .

9. Apothécies brun rougeâtre, à bord jaunâtre; thalle gris sale,

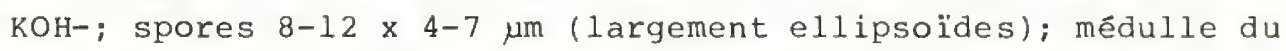
bord des apothécies (amphithécium) regorgeant d'algues. Taxon peu fréquent; s'établit sur les arbres le long des chemins et sur le bois............. . . . . . . . . L. saligna

9. Coloration du bord des apothécies et du thalle variant entre jaune et vert jaunâtre, $\mathrm{KOH} \pm$ jaunâtre; spores étroites,

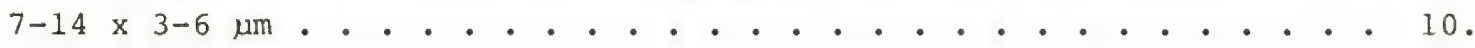

10. Bord des apothêcies disparaissant à maturitê, toujours lisse; amphithécium renfermant un petit nombre d'algues (apothécies d'aspect biatorines dans bien des cas); couleur des apothécies variant entre jaune et orange jaunâtre; thalle contenant de la zéorine. Taxon fréquent; corticole ou lignicole; vit particulièrement sur les conifères . . . . . . . . . . . . . . . . . . L. symmictera

10. Bord des apothécies normalement persistant; absence de zéorine . . . . . . . . . . . . . . . . . . 11.

11. Bord devenant granuleux ou sorédié; bord des apothécies qui ne renferme que quelques algues, couleur variant entre jaune et brun jaunâtre. Lichen commun; lignicole et corticole

11. Bord demeurant lisse; bord des apothécies qui regorge d'algues, très petites apothécies n'atteignant pas $0,25 \mathrm{~mm}$ de diamètre; coloration de leur disque variant entre brun jaunâtre et brun-rouge. Lichen rare; lignicole. . . . . L. piniperda

12. Thalle et bord des apothécies $\mathrm{KOH}+$ jaune (atranorine) . . . . 13 . 12. Thalle et bord des apothécies KOH- (absence d'atranorine) . . 20 . 
13. Épithécium coloré par des pigments brun-rouge clair (dans la partie supérieure de l'hyménium), non granuleux ni en surface, ni entre les extrémitês des paraphyses (fig. 18a) . . . . . 14 .

13. Epithécium pourvu de granulations de petite ou de grande taille disposées à la surface de l'hyménium (fig. l6a) ou entre les extrémités des paraphyses (fig. 17a); partie supérieure de l'hyménium pigmentêe ou presque entièrement hyaline, amphithécium renfermant des amas de gros cristaux incolores ( $\mathrm{fig} .16 \mathrm{~b}, 17 \mathrm{~b}$ ) (les cristaux et les granules de l'épithécium ont un grand pouvoir réfléchissant à la lumière polarisée) . . . . . . . . . . . . . . . . . . 17 .

14. Amphithécium contenant des amas d'énormes cristaux de forme irrégulière et insolubles dans KoH (les cristaux sont parfois épars); cortex se distinguant de la médulle de l'amphithécium, d'épaisseur uniforme; spores $(9,0-) 11,5-14,5(-16,5) \times(5,5-) 6,0-8,5 \mu \mathrm{m}$. Espèce rare; s'établi sur les arbres à feuilles caduques . . L. subfuscata

14. Amphithécium et cortex renfermant de minuscules cristaux anguleux et insolubles dans $\mathrm{KOH}$, remplissant ordinairement plus ou moins l'amphithécium (fig. 18b) . . . . 15 .

15. Cortex de l'amphithécium épais (tout particulièrement à sa base), gelatineux, ne se distinguant pas de la médulle (fig. $18 \mathrm{~b})$

15. Cortex de l'amphithécium mince ou absent, pas du tout gélatineux; spores $11-14 \times 7,0-8,5 \mu \mathrm{m}$. Lichen peu fréquent; crô̂t sur les arbres décidus... . . . . . . . . L. imshaugii

16. Petites apothécies devenant rapidement bombées de $0,3-0,6(-0,8) \mathrm{mm}$ de diamètre, fortement adnés au thalle; bord des apothécies lisse et mince; spores $9,5-14,5 \mathrm{x}$ $6,0-7,0(-8,0) \mu \mathrm{m}$. Taxon commun; pousse normalement sur l'écorce du hêtre ou de l'érable à sucre ............... (fig. 18,23) L. glabrata

16. Grandes apothécies plates, de $0,6-1,5(-2,0) \mathrm{mm}$ de diamètre, très resserrées à la base une fois à maturité; bord des fructifications bien visibles normalement flexueux; spores (12-)13-16(-19) x 7,5-10(-11) $\mu \mathrm{m}$. Taxon peu fréquent; s'installe sur l'écorce du peuplier et du frêne... . . . . . . . . . . . L. allophana 
(16)

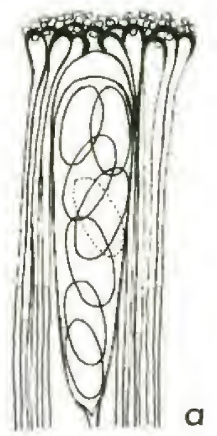

(17)

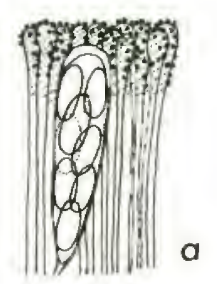

(18)

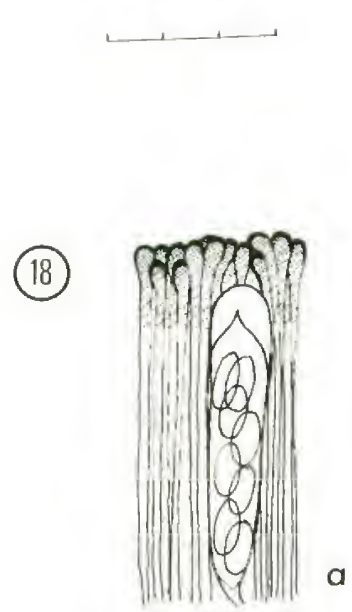

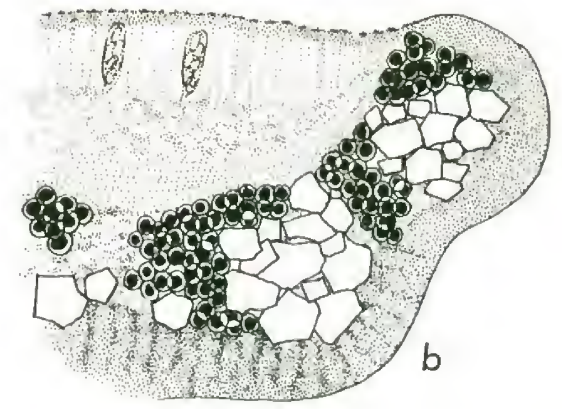
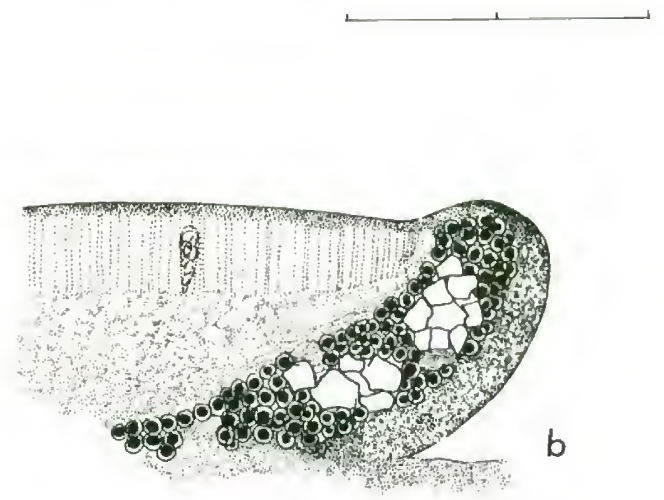

$b$
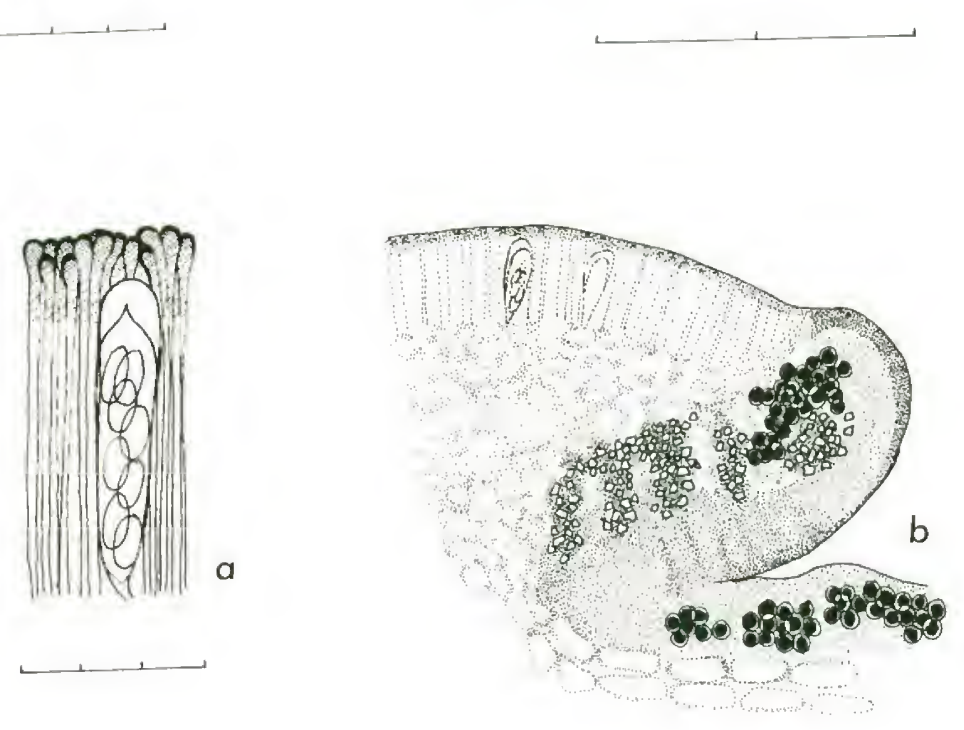

Figures 16 à 18.-16. Lecanora rugosella: a) fragment d'hyménium: épithécium granuleux en surface; b) coupe verticale d'une apothécie: l'amphithécium renferme des amas de gros cristaux.-17. Lecanora pseudochzarotera: a) fragment d'hyménium: fins granules entremêlés aux extrémités des paraphyses; b) coupe verticale de l'apothécie.-18. Lecanora glabrata: a) fragment d'hyménium: épithécium dépourvu de granules entremêlés aux extrémités des paraphyses, mais pigmenté; b) coupe verticale d'une apothécie; présence de petits cristaux dans l'amphithécium s'étendant du medulle au cortex. Échelle:-16a, 17a, 18a: chaque division $=10 \mu \mathrm{m} ;-16 \mathrm{~b}, 17 \mathrm{~b}, 18 \mathrm{~b}$ : chaque division $=100 \mu \mathrm{m}$. 
17. Apothécies d'abord enfoncées dans le thalle, devenant sessiles par la suite; bord verruqueux et discontinu; disque des fructifications brun nuancé d'orange ou brun-rouge, cortex des ascocarpes très mince (moins de $15 \mu \mathrm{m}$ ); difficilement distinguable; épithécium pàt orange, produisant des amas de petites aiguilles orange comme le révèle le microscope; spores $11,5-14,5 \times 7,5-8,5 \mu \mathrm{m}$. Espèce fréquente; s'établit sur les arbres decidus..... . L. cinereofusca

17. Toutes les apothécies sessiles; bord des fructifications entier, de temps en temps verruculeux; épithécium Pd-; cortex gélatineux, distinct . . . . . . . . . . . . . . . 18 .

18. Bord des apothécies Pd+ rouge (acide

fumar-protocétrarique); épithécium pigmenté, renfermant de fins granules entremêlés aux extrêmités des paraphyses. Taxon fréquent; rencontré particulièrement sur l'écorce des conifères . . . . . . . . . . L. pulicaris 18. Bord des apothécies Pdt jaune ou Pd- . . . . . . . . . 19.

19. Thalle verruqueux, épais, dont la coloration varie entre gris clair et presque blanc; constriction à la base des apothécies dont le bord est grossièrement verruqueux; disque ordinairement brun jaunâtre ou brun rosâtre, de temps en temps brun foncé, souvent légèrement pruineux chez les jeunes apothécies; épithécium grossièrement granuleux à la surface, non pigmentē (présence de pigments uniquement au bout des paraphyses); spores $12-18 \times 7-10(-11,5) \mu \mathrm{m}$. Espèce rare; habite surtout l'écorce de Thuja . . . . . (fig. 16) L. rugosella

19. Mince thalle gris cendré, verruculeux ou aréolé; apothécies sessiles et absence de constriction à la base; bord lisse à légèrement verruculeux; disque brun-rouge, pourvu de minuscules granules se mêlant aux extrémités des paraphyses; spores 10-13 x 6,5-8,5 $\mathrm{m}$. Taxon commun; corticole

................. (fig. 17) L. pseudochlarotera

(N.B.: L. chlarotera, espèce voisine dont l'épithêcium est parsemé de granules grossiers à la surface (comme chez L. rugosel(za), n'a pas été repéré avec certitude dans l'Outaouais; il s'agit d'une espèce rare en ontario.) 
20. Bord des apothécies épais, blanc; fructifications groupées, brun jaunâtre pâle, certaines d'entre elles étant légèrement pruineuses; cortex de l'amphithécium mince, indistinct, non gélatineux. Taxon rare, lignicole . . . . . . . . . . . . . L. umbrina f. gregaria (N.B.: Il s'agit peut-être d'un morphotype de $L$. hagenii.)

20. Bord des apothécies mince ou épais, jaunâtre, gris foncé ou concolore au thalle, pas blanc; teinte des fructifications allant du pâle au sombre . . . . . . . . . . . 21 .

21. Cortex du bord des apothécies distinct, gélatineux; apothécies presque biatorines, dont l'amphithécium contient quelques algues; mince bord concolore au disque; fructifications devenant immarginées. Taxon rare, lignicole

21. Cortex du bord des apothécies mince, indistinct, ni gélatineux, ni étendu; amphithécium regorgeant normalement d'algues; bord distinct, jaunâtre, habituellement persistant pendant longtemps . . . . . . . . . . . . . . . . . 22 .

22. Spores 8-12 × 4-7 um (forme d'une large ellipse); disque des apothécies brun-rouge; bord des fructifications épais, proéminent, de verruqueux à crênelé; apothêcies de 0,4-0,75 mm de diamètre dépourvues de pruine. Taxon peu fréquent; habite sur l'écorce de l'orme et le bois . . . . . . . . . . . L. saligna 22. Spores 7-12 × 3-4,5 um (forme d'une ellipse étroite); couleur des apothécies variant entre brun jaunâtre et brun très foncë; bord des fructifications mince, proéminent et allant de lisse à égal au disque; apothécies de $0,2-0,4 \mathrm{~mm}$ de diamètre, légèrement pruineuses. Taxon rare; lignicole . . . . . . . L. piniperda

23. Apothécies enfoncées dans le thalle, orange clair ou rose nuancé d'orange; thalle lisse à fendillé, uniforme. Lichen frēquemment retrouvé sur les pierres au moins submergẻes périodiquement . . . . . . . . . . . . . . Hymenelia lacustris

23. Apothécies sessiles; thalle non orange; lichens non aquatiques . . . . . . . . . . . . . . . . . . 24 . 
24. Thalle au contour nettement lobé, ou pourvu d'aréoles lobées; taxons vert jaunâtre (acide usnique) . . . . . . . . 25.

24. Thalle uniforme, ou constitué d'aréoles dispersées, non lobē . . . . . . . . . . . . . . . . . . . 28.

25. Apothécies pruineuses; médulle kOH- . . . . . . . . . . . . . . . 26. 25. Apothécies dépourvues de pruine . . . . . . . . . . . . . . . 27 .

26. Apothécies orange rosâtre ou orange jaunâtre; thalle terne, vert jaunâtre pâle, souvent presque ombiliqué; prêsence d'acide pseudoplacodialique. Lichen fréquent, colonisant les roches granitiques... . . . L. chrysoleuca s. . 26. Apothécies vert jaunâtre, thalle brun-jaune brillant, aréolé à légèrement lobé, présence d'acides gras.

Lichen rare; habite les roches granitiques. . L. cf. subdiscrepens

27. Médulle KOH+ jaune (substance inconnue); apothécies variant de jaunâtre à chamois jaunâtre; thalle composé d'arêoles dispersếes, mal fixées et lobées; présence d'acide placodialique et d'une substance inconnue. Taxon peu fréquent; s'installe sur le granite . . . . . . . . . . L. opiniconensis

27. Médulle KOH-; apothécies vert-jaune à brun jaunâtre; thalle formé de lobes à disposition radiale, fortement appliqué contre le substratum; présence de zéorine. Taxon commun; colonise les roches calcaires, et les roches siliceuses si ces dernières servent de perchoir aux oiseaux . . . . . . . . L. muralis

28. Thalle ou apothécies nettement jaunâtre ou vert

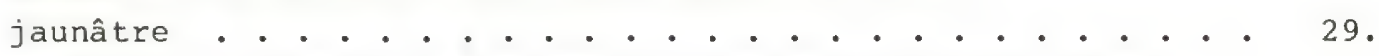
28. Thalle et apothécies depourvues de toute teinte de jaune . . . . . . . . . . . . . . . . . . . . . . . . . . .

29. Apothécies pruineuses, vert jaunâtre, épais thalle aréolé; présence d'acides gras ( $v$. doublet 26) . . . . . ‥ L.

29. Apothécies dépourvues de pruine, vert jaunâtre, la plus grande partie du thalle est d'habitude absente; présence de zéorine. Lichen fréquent, colonisant les roches non calcaires . . . . . . . . . . . . . . . . . . . L. polytropa

30. Thalle et bord des apothécies KoH+ jaune (atranorine). Taxons habitant les roches non calcaires . . . . . . . . . 31 . 
30. Thalle et bord des apothécies KOH- . . . . . . . . . . . . 34 .

(N.B.: Si le thalle est C+ rose et $\mathrm{KOH}-$, on a peut-être

affaire à Trapelia involuta. Consulter le doublet

13 dans la clef des Lecidea.)

31. Disque des apothécies pruineux; épithécium fortement

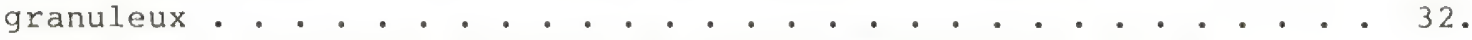

31. Disque des apothécies dépourvu de pruine; épithécium pas du tout granuleux . . . . . . . . . . . . . . . . . 33 .

32. Disque des apothécies fortement pruineux, $C+$ jaune (sordidone); jeunes apothécies souvent enfoncées dans le thalle; bord des fructifications ne contenant pas de gros cristaux. Espèce rare . . . . . . . . . . . L L rupicola

32. Disque des apothécies légèrement pruineux, C-; présence d'un acide gras; Eructifications saillantes, souvent entourées d'un bord verruqueux; bord renfermant des amas de gros cristaux d'oxalate. Espèce peu fréquente. . . L.

33. Disque des apothécies variant de brun foncé à noir; cortex du bord des fructifications épais (atteingnant 65 um à la base), rayonnant, à l'intérieur duquel de minuscules granules distincts sont dispersés; parfois absence de gros cristaux dans le bord; présence de gangaléoidine et d'une substance inconnue. Taxon peu fréquent . . . . . . . . . . . . . . L. sp. \#1 (N.B.: Peut-être est-il apparenté à $L$. cenisia ou gangaleoides.)

33. Disque des apothécies brun-rouge; bord mince ou épais, crénelé; cortex du bord des fructifications mince, 15-25 Mm, distinct ou non, non rayonnant; gros cristaux dans l'amphithêcium; présence d'une substance non identifiée.

Taxon fréquent. . . . . . . . . . . . . . . . . . galactinula

34. Espèces qui poussent sur les roches calcaires; thalle

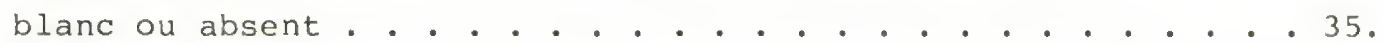

34. Espèce qui pousse sur des roches non calcaires; thalle brun grisâtre terne, fendillé-aréolé; spores 9-10 x 4,5-5,5 $\mu \mathrm{m}$. Lichen rare. . . . . . . . . Lecanora sp. \#2 
35. Disque des apothécies noir, couvert d'une pruine grise; thalle souvent bien visible, blanc, aréolé; spores adoptant la forme d'une ellipse étroite, $11,0-13,5 \times 5,0-6,5 \mu \mathrm{m}$. Lichen peu fréquent. . . . . . . . . . . . . L. crenulata

35. Disque des apothécies brun-rouge ou brun jaunâtre, sans pruine (ou chez les jeunes, revêtu d'une mince pruine); absence du thalle; spores ellipsoïdes, $8,5-10,5 \times 5,2-6,5 \mu \mathrm{m}$. Lichen fréquent............... . . L dispersa

\section{LECIDEA ET LECIDELLA (espèces fertiles)}

1. Espèces lignicoles, corticoles, muscicoles ou terricoles . . . . 2 .

1. Espèces saxicoles . . . . . . . . . . . . . . . . 11.

2. Lichens corticoles .. . . . . . . . . . . . . . . 3 .

2. Lichens lignicoles, muscicoles ou terricoles......... . 6 .

3. Thalle sorédié, amas ronds et distincts de sorédies vert jaunâtre disposés sur une croûte mince et continue; apothécies rares, brun pâle, les tissus entièrement hyalins; spores 11-13 x 2,5-3,5 $\mu$, étroitement ellipsoïdes à fusiformes. Rare; pousse au pied des arbres, du frêne surtout. . . . . . Lecidea sp. (no 4 au sens de Harris, 1977)

3. Thalle continu, mince ou épais, lisse ou granuleux, dépourvu de soralies distinctes . . . . . . . . . . . . . . 4 .

4. Très petites apothécies planes, de 0,18-0,40 mm de diamètre, souvent groupées, brun-rouge; mince thalle $\mathrm{KC}+$ orange pâle (de temps en temps $\mathrm{KC}-$ ); hypothécium hyalin; spores 10-13 x 5,2-7,0 um, de la forme d'une large ellipse. Espèce fréquemment retrouvée sur les arbres à feuilles caduques . . . . . . . . . . . . L v varians

4. Apothécies de planes à bombées, de 0,2-0,75 $\mathrm{mm}$ de diamètre, éparpillées, dont la couleur varie entre brun jaunâtre et marron ou noir; spores adoptant la forme d'une ellipse étroite . . . . . . . . . . . . . 5.

5. Couleur des apothécies s'échelonnant généralement entre brun foncé et noir (exceptionnellement pâles), ces dernières variant entre une forme plus ou moins plane et un aspect 
légèrement bombé, entourêes d'un mince bord luisant;

hypothêcium brun foncé; thalle très mince, uniforme, spores

$10-13,5 \times 3,0-4,0 \mu \mathrm{m}$. Lichen fréquent; habite sur différents

arbres . . . . . . . . . . . . . . . . L . albofuscescens

5. Couleur des apothécies s'échelonnant entre brun jaunâtre et

brun-rouge, apothécies convexes à globuleuses; bord

disparaissant rapidement; coloration de l'hypothécium variant

entre la transparence et un jaune sale pâle; thalle

vert-de-gris, granuleux; spores $15-17(-23) \times 4,0-6,0 \mu \mathrm{m}$.

Lichen peu fréquent; s'établit sur un grand nombre d'arbres,

près de leur base. . . . . . . . . . . . . . . L v vernalis

6. Couleur du thalle, variant entre brun foncé et noir, thalle granuleux,; apothécies noires, immarginées, pourvues d'un hypothécium et d'un rebord propre brun foncé; spores $10-15 \times 5-7(-9) \mu \mathrm{m}$. Taxon peu fréquent; habite sur les sols sablonneux ou tourbeux, ou encore sur le bois en décomposition . . . . . . . . . L. uliginosa

6. Couleur du thalle s'échelonnant entre gris et vert-de-gris, ou jaunâtre . . . . . . . . . . . . 7.

7. Thalle gris à vert-de-gris . . . . . . . . . . . . . . 8 .

7. Thalle jaunâtre; apothécies jaune clair (v. clef des

Lecanora, doublet 10) . . . . . . . . . Lecanora symmictera

8. Épais thalle, vert-de-gris, verruqueux, dont certaines

verrues deviennent sorédiées, ct rose (acide

gyrophorique); apothécies de forme irrégulière, planes à

convexes, dont la coloration s'étend du rose terne au

noir brunâtre ou au noir grisâtre; spores $9-13 \times 4-6$

um. Frëquent; terricole ou lignicole... . . . . L. granulosa

8. Thalle mince ou épais, non sorédié, C-; apothécies brunes ou noir ébène . . . . . . . . . . . . . . . . . . 9.

9. Apothécies noir ébène, luisantes, aplanies, irrégulières, entourées d'un mince bord; épais thalle verruqueux, KOH+ jaune, Pd+ rouge (substance non identifiée); spores 5,7-7,8

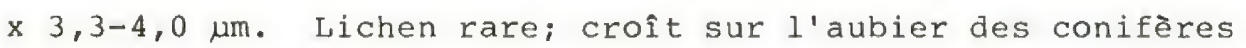
. . . . . . . . . . . . . . . . . . . . L. elabens 
9. Apothécies brun-rouge ou brun jaunâtre; apothécies bombées, rondes; thalle plus ou moins verruculeux à granuleux; longueur des spores dépassant $10 \mu \mathrm{m}$. . . . . . . . . . . 10 .

10. Hypothécium brun clair ou foncé, plus pâle en dessous; spores $10-14(-17) \times 3,6-4,8 \mu \mathrm{m}$. Taxon fréquent; pousse sur les mousses au pied des arbres... . . . . L. berengeriana

10. Couleur de l'hypothécium variant entre la transparence

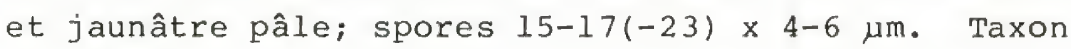
peu fréquent, muscicole, lignicole et corticole . . . L. vernalis

11. Espèce colonisant les roches calcaires; thalle épais, gris ou mince, voire absent; apothécies rondes, à bord épais et plat; coloration de l'hypothécium s'échelonnant entre la transparence et rose terne; rebord propre érugineux à l'extérieur (fig. 74 a); épithécium noir à noir olivâtre;

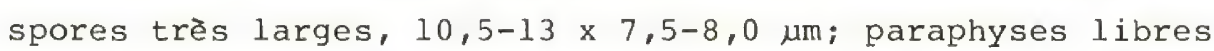
dans l'eau ou au moins dans $\mathrm{KOH}$. Espèce commune. . Lecidella stigmatea

11. Espèce s'établissant sur les roches non calcaires; thalle épais ou mince; hypothécium noir brunâtre, se fondant généralement plus ou moins avec le rebord propre . . . . . . . . 12 .

12. Très grandes spores, 13-24 x 6-11 um ........... 13 . 12. Spores plus petites, $6-13 \times 3-7 \mu \mathrm{m}$. . . . . . . . . 15 .

13. Thalle ct rose (acide gyrophorique), de morphologie variable: aréolé à dispersé, et alors verruqueux; mince rebord propre brun, parfois enveloppé d'un "bord thallin" secondaire; hypothécium hyalin; spores 17-22 x 8-10 $\mu \mathrm{m}$. Lichen peu fréquent . . . . . . . . . . . . . . Trapelia involuta

13. Thalle C-; rebord propre bien développé, normalement épais, dont la couleur s'échelonne entre brun foncé et noir, et carbonacé; hypothécium noir brunâtre... . . . . . . . . . . 14 .

14. Rebord propre à disposition radiale, noir brunâtre, paraplectenchymateux (V. Eig. 20); quelques spores, surtout des jeunes, pourvues d'une épispore ou "halo" (v. fig. 72a). Taxon commun....... (v. clef des Huilia) 
14. Rebord propre uniformément carbonacé, à disposition non radiale; bord des apothécies se fendillant souvent; thalle membraneux, gris à évanescent. Taxon rare . . . . . . . . . . . . . . . . L $\underline{\text {. cinereoatra }}$

(N.B.: L. subsimplex est aussi une possibilité.)

15. Épithécium verdâtre ou érugineux; rebord propre hyalin à l'intérieur, noir verdâtre au bord (fig. 19); très petites apothécies de 0,16-0,25 mm de diamètre; spores $6,1-7,5 \times$ $3,2-3,5 \mu \mathrm{m}$; thalle vert-de-gris foncé. Espèce fréquente; vit surtout sur les cailloux... . . . . . . . . . . . . L erratica

15. Épithécium brun; intérieur du rebord propre brun, extérieur pâlissant; apothécies de 0,3-0,5 mm de diamètre; spores 11-13 x 5-7 $\mu \mathrm{m}$; mince thalle uniforme. Espèce rare . . . . . L. delincta

\section{MICAREA}

1. Spores non cloisonnées . . . . . . . . . . . . . . . . . . . 2 .

l. Spores à $1-3$ cloisons; thalle C- . . . . . . . . . . . . . . . . . 3 .

2. Espèce qui pousse sur le bois décomposé. Couleur du

thalle qui varie entre vert-de-gris clair et vert

brunâtre, thalle grossièrement granuleux à sorédié et

mal délimité, C+ rose (acide gyrophorique); ascocarpes

noirs, groupés ou agglomérés; spores 6-10(-12) x 3-4(-5)

$\mu$ m. Peu fréquent . . . . . . . . . . . . . . M. viridescens

2. Espèce qui s'installe sur le roc dans les stations

ombragées. Thalle gris verdâtre, continu à

fendillé-aréolé, qui devient finalement granuleux, C- ; ascocarpes noirs à brun verâtre, éparpillés, de 0,2 $0,4 \mathrm{~mm}$ de diamètre; hypothëcium hyalin; spores 9-13 $\mathrm{x}$ 3,3-4,3 um. Rare . . . . . . . . . . . . . M. bauschiana

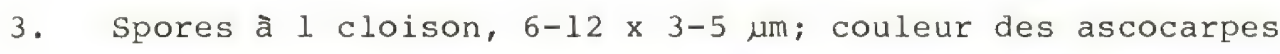
variant entre rose et jaune terne, ascocarpes de $0,1-0,3 \mathrm{~mm}$ de diamètre, groupés; thalle verdâtre, pulvérulent. Taxon rare, corticole ou, exceptionnellement, sur l'aubier dur 


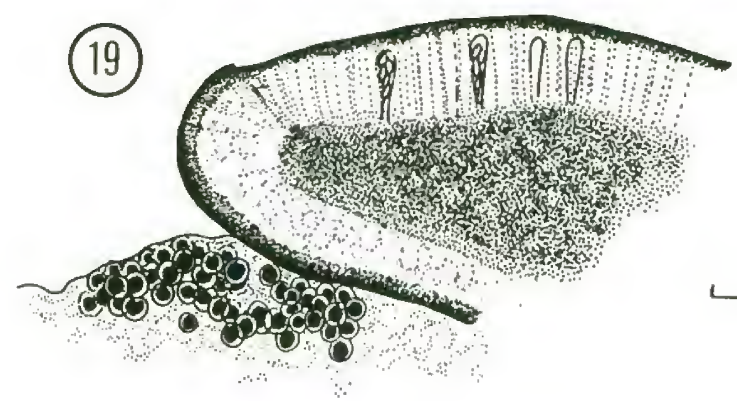

(20)
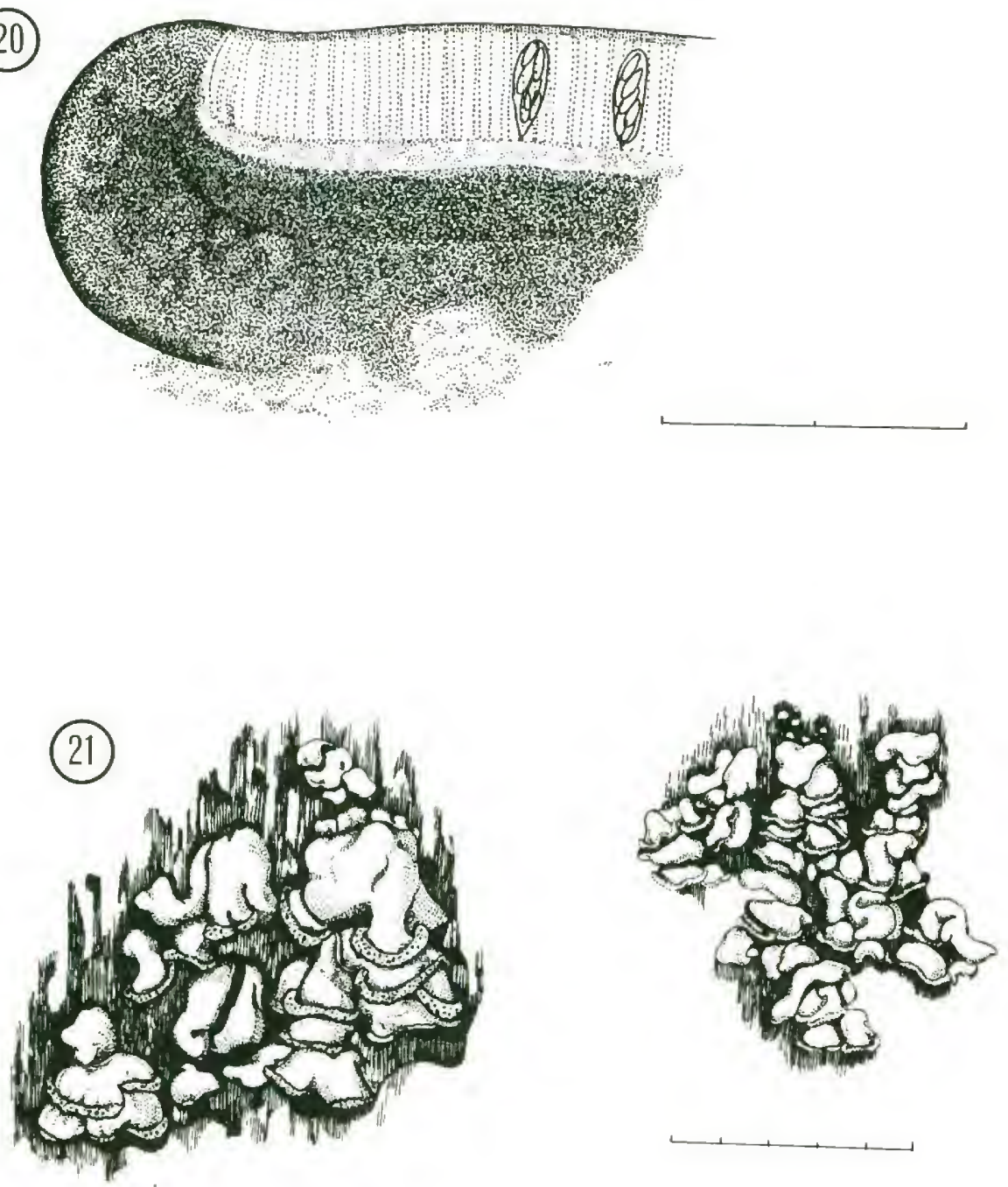

Figures 19 à 21.-19. Lecidea erratica: coupe verticale d'une apothécie. -20. Huilia macrocarpa: coupe verticale d'une apothécie. -21 .

Hypocenomyce scalaris. Échelle: $-19,20$ : chaque division $=100 \mu m$; -21: chaque division $=1 \mathrm{~mm}$. 


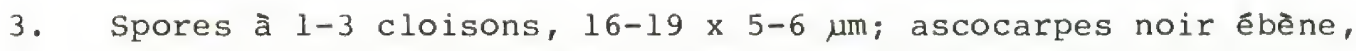
pour la plupart dispersés; couleur du thalle variant entre vert foncé et noir verdâtre, thalle lisse ou de verruculeux à granuleux. Taxon peu fréquent; pousse ordinairement sur les tronçons d'arbre et le bois en décomposition . . . . . . M. melaena

OCHROLECHIA

1. Thalle couvert de soralies distinctes, stérile; soralies C+ rose (acide gyrophorique) . . . . . . . . . . . . . . . 2 .

1. Thalle non sorédié, fertile... . . . . . . . . . . . . 3 .

2. Soralies UV+ orange (lichexanthone), finement granuleuses ou farineuses; thalle mince. Lichen fréquent; corticole . . . . . . . . . . . . o. arborea

2. Soralies UV-, faites de très grosses granulations; thalle épais. Lichen rare; muscicole, épiphyte ou s'établit exceptionnellement au pied des arbres . . ‥ androgyna

3. Thalle et bord des apothécies C-; disque C+ rose; un morceau de l'apothécie dans KоH donne de longs cristaux en forme d'aiguille (acide variolarique). Taxon rare; corticole . . . . . . . . . . . . . . . . . o. pseudopallescens

3. Thalle et bord des apothécies c+ rose (acide gyrophorique); absence de l'acide variolarique. Taxon fréquent; corticole orosella

\section{PERTUSARIA}

1. Verrues fructifères sorédiées ou lécanorines (entourées d'un épais bord reconnaissable) . . . . . . . . . . . . . . . 2 .

1. Verrues fructifères s'ouvrant par un ou quelques ostioles, ni sorédiées, ni lécanorines (v. fig. 24,74a) . . . . . . . . . . . 7 .

2. Verrues fructifères sorédiées . . . . . . . . . . . . . . . 3 . 2. Verrues fructifères lécanorines; une spore par asque . . . . 6 .

3. Verrues sorédiées et médulle KC+ pourpre, K-, C-, Pd- (acide

picrolichénique); thalle gris foncé, grossièrement granuleux, 
pourvu de saillies sorédiées. Lichen peu fréquent; corticole

3. Verrues sorédiées et médulle $\mathrm{kC}-$. . . . . . . . . . . . . 4.

4. Sorédies $\mathrm{K}-, \mathrm{Pd}-, \mathrm{C}-$; une spore par asque (fréquemment

absent). Taxon fréquent; corticole... . . . .

4. Sorêdies Pdt orange ou rouge . . . . . . . . . . . . . 5.

5. Sorédies $\mathrm{KOH}+$ jaune vif, Pd+ orange jaunâtre (acide

thamnolique); deux spores par asque. Espèce rare; corticole

. . . . . . . . . . . . . . . . . . . p. trachythallina

5. Sorédies KOH-, Pd+ rouge (acide fumar-protocétrarique); une

spore par asque. Espẽce peu fréquente, corticole. . P. multipunctoides

6. Disque $\mathrm{C}-, \mathrm{KOH}+$ rouge, $\mathrm{Pd}+$ jaune (acide norstictique);

disque noir, plus ou moins pruineux; spores 95-142 x

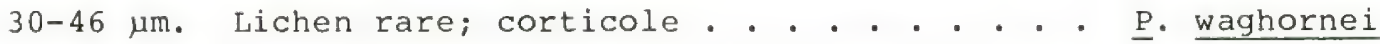

6. Disque C+ rouge, Кон-, Pd- (acide lécanorique); disque

revêtu d'une épaisse pruine grise; spores 180-274 x

47-81 um. Lichen peu fréquent; corticole....... . p. velata

7. 4 - 8 spores par asque . . . . . . . . . . . . . . . . . 8 .

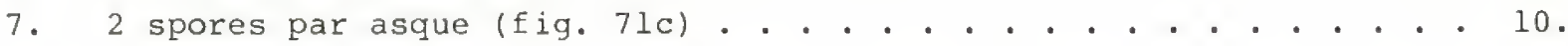

8. Spores unisériées; médulle (surtout dans les verrues)

Pdt orange clair, KoH+ jaune (acide stictique) . . . . . . . . 9.

8. Spores bisériées; cortex et médulle pd+ jaune, koH+

jaune qui vire au rouge sang (acide norstictique);

spores $6-8$ par asque, $72-100 \times 28-38 \mu \mathrm{m}$. Peu

fréquent; pousse sur les feuillus. . . . . . . . . $\underline{\text { P rubefacta }}$

9. Spores au nombre de 8 par asque, 36-60 x 18-32 um. Taxon peu

fréquent; corticole. . . . . . . . . . . . . . . p. alpina

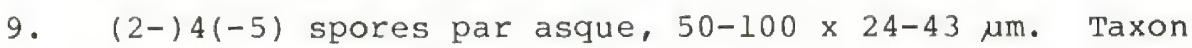

fréquent; corticole . . . . . . . . . . . . . . . P. leucostoma

10. Médulle KoH+ jaune qui tourne au rouge, Pd+ jaune

(acide norstictique); parois des spores rugueuses;

épithécium кон-. Espèce rare; corticole... . . . ‥ neoscotica

10. Médulle KoH+ jaune, Pd+ orange clair (acide stictique)... . 11. 
(22)

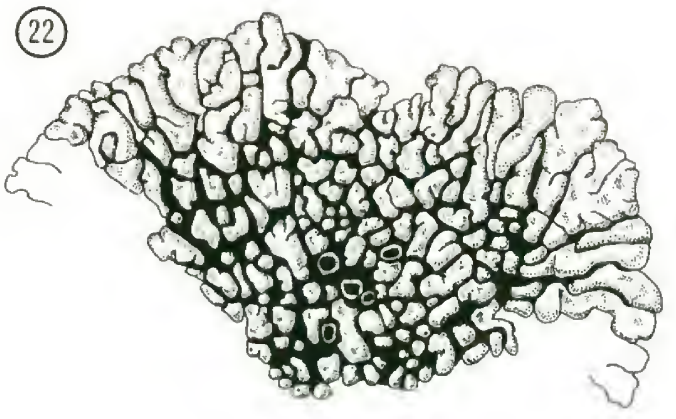

(23)

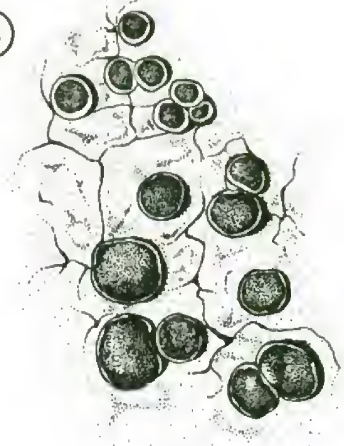

(25) 8

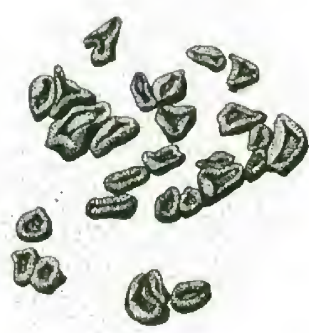

(24)
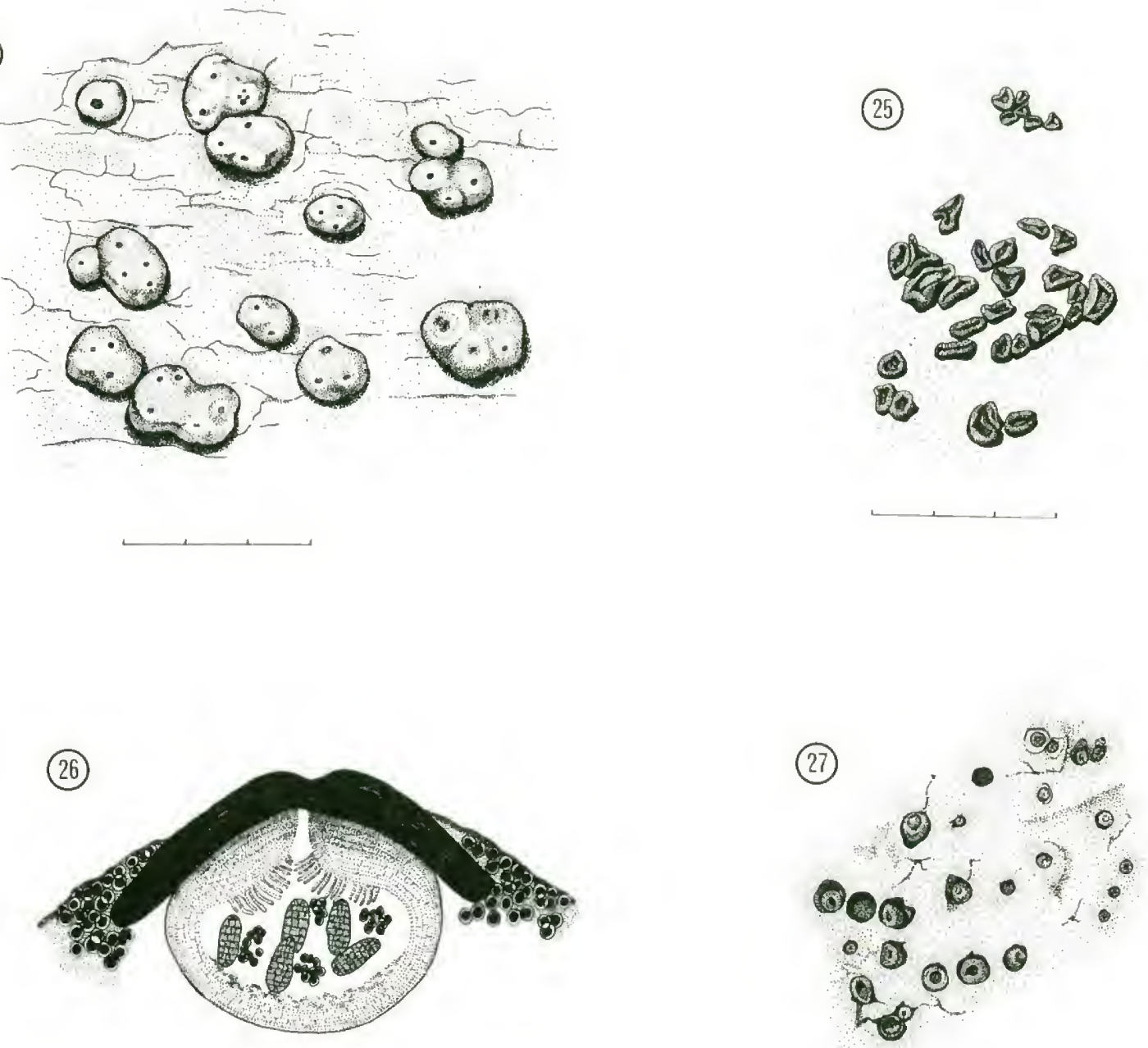

(27)

Figures 22 à 27.-22. Dimelaena oreina.-23. Lecanora glabrata.-24. Pertusaria macounit.-25. Sarcogyne simplex.-26. Staurothele fissa: coupe verticale d'un périthẽce. -27 . Verrucaria calciseda. Échelle:-22, 23, 24, 25, 27: chaque division $=1 \mathrm{~mm} ;-26$ : chaque division $=100 \mu \mathrm{m}$. 
11. Épithécium KOH+ pourpre; parois des spores entièrement lisses, KOH-. Lichen rare; corticole... . . . . . P. consocians

11. Épithécium KOH-; parois des spores striées radialement de façon nette, (fig. 5n), KOH+ pourpre spores vertes. Lichen commun; corticole.............. (fig. 24) $\underline{\text { p }}$ macounii

\section{PLAG IOCARPA}

(N.B.: Dimensions des spores tirées de Harris, 1973)

1. Spores hyalines, à trois cloisons, $18-27(-30) \times 7-10(-12) \mu \mathrm{m}$ . . . . . . . . . . . . . . . . . . . p. hyalospora

1. Spores brun foncé . . . . . . . . . . . . . . . . . . 2.

2. Spores à 3 cloisons, 30-40(-45) x (12-)14-16(-18) $\mu \mathrm{m}$

2. Spores à 7 cloisons................ 3

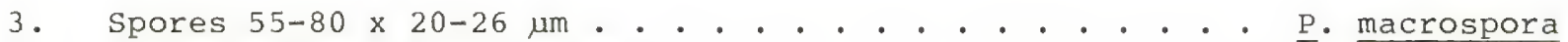

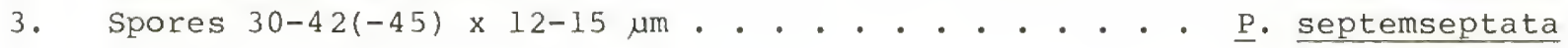

PSORA ET HYPOCENOMYCE

1. Lichen terricole ou calcicole; épaisses squamules, dont la couleur varie de brun à brun rouge, au bora blanc; médulle et cortex Pd- et C-. Lichen peu frëquent....... . Psora russellii

1. Lichen lignicole, s'installant d'habitude sur le bois carbonisé ou, exceptionnellement, sur l'écorce . . . . . . . . . 2.

2. Squamules sorédiées au bora de la face inférieure . . . . . . 3.

2. Squamules sans sorédies, très petites, Pd-, C-, d'habitude fertiles et pourvues de petites apothécies noires. Taxon peu fréquent....... Hypocenomyce friesii

3. Squamules olive clair, très bombées, Ct rouge, Pd- (acide lécanorique). Espèce fréquente... (fig. 2l) Hypocenomyce scalaris

3. Squamules olive qui tire sur le brun, ordinairement plus ou moins planes, C-, $\mathrm{Pd}+$ rouge (substance inconnue). Espèce

rare... . . . . . . . . . Hypocenomyce anthracophila 
PYRENULA

(N.B.: Dimensions des spores tirées de Harris, 1973)

1. Spores 13-24 um de long. . . . . . . . . . . . . . . . . . . 2.

1. Spores 22-35(-46) um de long . . . . . . . . . . . . . . . . . 3 .

2. Couleur du thalle qui s'échelonne entre blanc et gris, UV-; hyménium dépourvu de gouttelettes d'huile; spores $13-19(-21) \times 8-9(-11)$ um, les parois des cellules du bout sont très distinctes. Rare; pousse sur les essences à feuilles caduques . . . . . . . . . . . .

2. Couleur au thalle qui s'échelonne entre olive et olive jaunâtre, UV+ jaune; gouttelettes d'huile dispersées en grand nombre dans l'hyménium; spores 13-22(-24) x 7-12 $\mu \mathrm{m}$, parois des cellules du bout très minces, ce qui fait que les cellules sont saillantes, un peu comme des papilles. Fréquent; pousse sur l'écorce tendre, surtout celle du hêtre, du chêne et de l'érable . . . . . . . P. neglecta

3. Thalle parsemé de minuscules points blancs; présence de gouttelettes d'huile dans l'hyménium; spores $24-35(-46) \mathrm{x}$ 10-15(-17) um. Rare . . . . . . . . . . . . . … imperfecta

3. Thalle dépourvu de points blancs; absence de gouttelettes

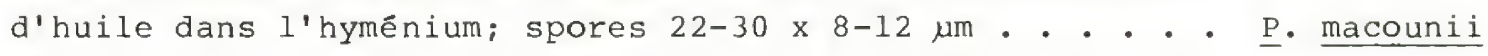
(N.B.: Il n'a pas encore été trouvé dans l'outaouais, mais il a été décrit à partir de spécimens récoltés dans le sud de l'ontario; il pousse sur l'écorce tendre, p. ex. celle de Carpinus, encore appelé le charme de Caroline.)

\section{RHIZOCARPON}

1. Thalle jaune verdâtre, pourvu d'un hypothalle noir et bien visible; aréoles en forme de croissant ou verrues bordant en partie les apothécies enfoncées dans le thalle; médulle IKI+ bleu; spores brun verdâtre très foncé, 30-38 x 12-16 um, murales, pluricellulaires. Taxon qui colonise les roches granitiques exposées . . . . . . . . . . . . . . . R lecanorinum (N.B.: Cette espèce, quoiqu'elle n'ait pas encore été signalée dans l'Outaouais, devrait, selon toute attente, s'y trouver.) 
1. Couleur du thalle qui varie entre gris et brun ou, parfois, plus ou moins orange . • . . . . . . . . . . . . . . . . . . 2 .

2. Spores à $1-3$ cloisons (non murales), hyalines . . . . . . . 3 .

2. Spores murales . . . . . . . . . . . . . . . . . . 4 .

3. Spores uniseptêes, 18-24 x 8-10 $\mu \mathrm{m}$; thalle uniforme,

fendillé, dont la couleur varie entre brun et gris brunâtre;

apothécies sessiles, à épais bord persistant. Espẽce peu

Eréquente . . . . . . . . . . . . . . . . . ‥ hochstetteri

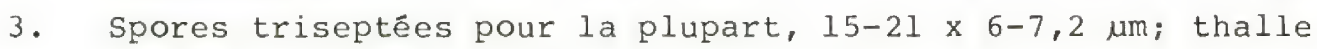
aréolé, brun foncé; apothécies enfoncées dans le thalle. Lichen rare . . . . . . . . . . . . . . . . . .

4. Thalle koH+ rouge, Pd+ jaune (acide norstictique);

thalle gris clair, aréolé-verruqueux; apothécies sessiles, à épais bord proêminent; spores hyalines, qui foncent en vieillissant, 20-25(-34) x 8,5-11(-16) um. Taxon rare

R. eupetraeum

4. Thalle $\mathrm{KOH}-$ ou $\mathrm{KOH}+$ jaune, $\mathrm{Pd}-$; coloration thalline variant entre gris et brun . . . . . . . . . . . . . . . 5 .

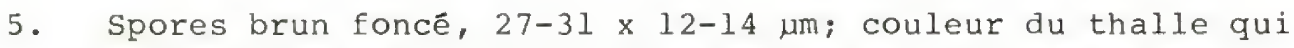
varie entre brun rosâtre et brun grisâtre foncé, thalle épais, arêolé-verruqueux, pourvu d'apothêcies bombêes et immarginêes, situées entre les aréoles; médulle IKI+ bleu, cortex C+ rose (acide gyrophorique). Espèce fréquente; colonise les roches exposés . . . . . . . . . . . . . . R grande

5. Spores hyalines; thalle aréolé, plat; en règle gênérale, apothécies entourées d'un bord gris et persistant; médulle IKI-, cortex $\mathrm{C}-$. . . . . . . . . . . . . . . . . . . . 6 .

6. Spores à 5-7(-9) cloisons transversales et $1-2$ cloisons longitudinales en général; 23-35 x 10-15 $\mu \mathrm{m}$; couleur du thalle variant entre brun rosâtre clair et brun grisâtre ou ocracé (orange); apothêcies insérẻes entre les aréoles. Lichen fréquent; saxicole, rencontré dans les cours d'eau ou à proximité . . . . . . . . . . . . . 1 . lavatum

6. Spores à 3-4 cloisons transversales et 1 cloison

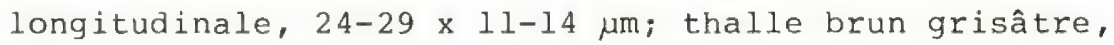


continu à fendillé; apothécies sessiles ou \pm enfoncées. Lichen peu fréquent; colonise les rochers secs. . $\underline{\text { R. obscuratum }}$

RINODINA

1. Thalle saxicole. . . . . . . . . . . . . . . . . . 2 .

l. Thalle corticole ou lignicole. . . . . . . . . . . . . 5.

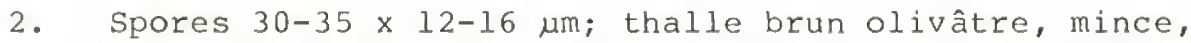
membraneux, devenant presque squamuleux en partie, KOH-; apothécies à épais bord persistant, de 0,50-0,75 mm de diamètre. Taxon rarement rencontré sur le roc (plus fréquemment sur l'écorce) . . . . . . . . . . . R. ascociscana 2. Spores n'atteignant pas 25 um de long et 12 um de large.... 3.

3. Spores entourées au centre d'une bande nettement sombre (fig. 28), 16-20 x 9,5-11,5 um; petites apothécies de 0,25-0,33 mm de diamètre, noires; bord foncé ou gris, mince; thalle absent ou gris et non uniforme. Espèce rare; vit sur le calcaire . . . . . . . . . . . . . . . . . . . . .

3. Parois des spores uniformément pigmentées, dépourvues de bande; thalle épais . . . . . . . . . . . . . . . . . . . 4.

4. Thalle blanc, verruqueux, KOH+ jaune; grandes apothécies de 0,4-0,8 mm de diamètre, à épais bord inégal et crénelé (qui disparaît parfois ce qui confère aux ascocarpes un aspect lécidéin); spores 22-23 x 10,5-12 $\mu \mathrm{m}$, dépourvues de cloisons évidentes. Lichen fréquent; calcicole . . . . . . . . . . . . . . . . R. verrucosa

4. Thalle brun olivâtre clair, aréolé à squamuleux, KoH- ; apothécies de $0,4-0,5 \mathrm{~mm}$ de diamètre, saillantes, entourêes d'un bord blanc, mince ou épais; spores 18-26

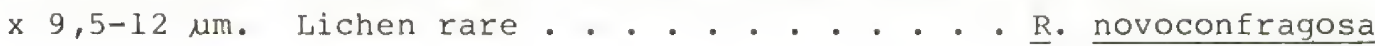

5. Spores dépassant $25 \mu \mathrm{m}$ de long (v. doublet 2) . . . . . R. ascociscana 5. Spores n'atteignant pas $25 \mu \mathrm{m}$ de long . . . . . . . . . . . . . 6 .

6. Spores au nombre de 8 par asque, dépassant généralement

$16 \mu \mathrm{m}$ de long. . . . . . . . . . . . . . . . . . . . 7 . 
6. Spores au nombre de 12-32 par asque, n'atteignant pas 16 um de long en général . . . . . . . . . . . . . . . 9.

(28)

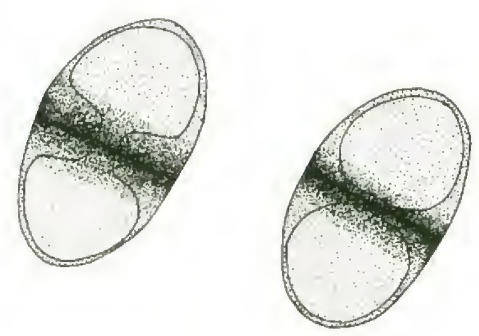

(29)

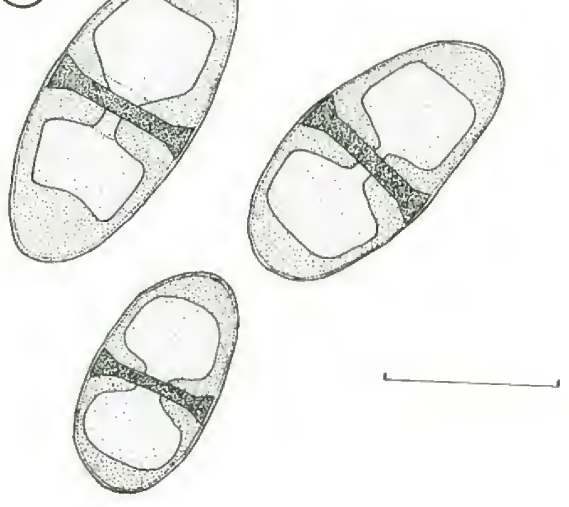

(30)

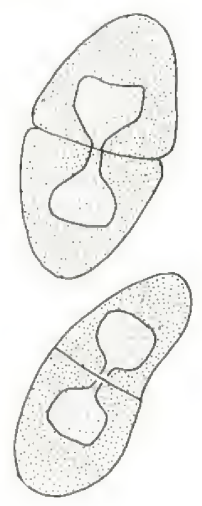

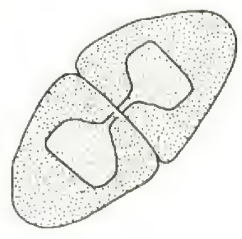

Figures 28 à 30 : ascospores. 28. Rinodina bischoffii. 29. Rinodina dakotensis. 30. Rinodina halei. Echelle: chaque division $=10$ $\mu \mathrm{m}$.

7. Apothécies à épais bord égal à crénelé, de 0,33-0,66 mm de diamètre; thalle verruculeux, dont la couleur varie entre gris verdâtre et brun terne; spores 16-20 x 6,5-10 $\mu \mathrm{m}$, septum brun nettement plus foncé (Fig. 29). Fréquent; corticole

R. dakotensis

7. Apothécies saillantes, souvent entourées d'un mince bord échancré faisant office de thalle, membraneux; septum des spores distinct, mais non bruni (fig. 30) . . . . . . . . . . . 8 .

8. Thalle blanc; spores $13,5-20 \times 7,5-9,5 \mu \mathrm{m}$; apothécies de 0,33-0,50 mm de diamètre; espèce fréquente, croissant sur l'écorce d'Acer, de Tilia et de Fraxinus

R. subminuta

8. Thalle gris tirant sur le vert; spores $15-21 \mathrm{x}$ $8,0-10,5(-11,5) \mu m ;$ apothécies de $0,25-0,41 \mathrm{~mm}$ de diamètre. Espèce fréquente. . . . . . . . . . . R. halei 
9. Spores au nombre de 12-16 par asque, $14,2-15,8$ x 6,8-7,4 $\mu$; parois des spores d'épaisseur inégale (fig. $72 \mathrm{~m}$ ); cortex des apothécies à peine visible, brun terne, d'environ $15 \mu \mathrm{m}$ d'épaisseur; bord des ascocarpes mince, finissant par disparaître et les fructifications deviennent convexes; thalle blanc, mince, membraneux. Lichen rare; pousse sur l'écorce de peuplier . . . . . . . . . . . . . . . ‥ polyspora

9. Spores au nombre de 16-32 par asque, 11-12 x 6 m; parois des spores d'épaisseur égale (comme dans Buelzia; v. fig. 722); cortex des apothécies bien visible, d'environ $65 \mu \mathrm{m}$ d'épaisseur; bord des ascocarpes épais, égal, persistant; thalle gris brunâtre, verruculeux. Lichen peu fréquent; croît sur l'ecorce de peuplier et d'orme . . . . . . . . . R. palustris (N.B.: R. populicola est une espèce voisine: les parois des spores sont d'épaisseur égale, par contre le nombre de spores par asque est $12-16$ et le thalle est vert grisâtre, parfois évanescent.)

\section{SARCOGYNE}

1. Lichen qui colonisant les roches calcaires (p. ex., le calcaire); apothécies pruineuses, de $(0,4-) 1,0-2,0 \mathrm{~mm}$ de diamètre, à bord mince mais distinct; rebord propre brun foncé, non carbonacé; mince thalle qui vit à la surface du substrat, ou incorporé à celui-ci et invisible. Taxon fréquent . . . . . . . . . . . . . . . . . . . s. regularis

1. Lichen qui s'installe sur les roches non calcaires (p. ex., le granite, le gneiss); apothécies non pruineuses; rebord propre carbonacé; thalle gênéralement invisible . . . . . . . . . . . 2 .

2. Apothécies de 1-2 mm de diamètre, rondes, normalement éparpillées, à bord assez lisse d'ordinaire et égal, parfois proéminent; disque lisse, marron (surtout une fois mouillé), \pm plat. Espèce peu fréquente . • • • . • $\underline{\text { s }}$ clavus

2. Apothécies de moins de 1 mm de diamètre, anguleuses, à bord généralement irrégulier et parfois discontinu . . . . . . . 3.

3. Disque des apothécies composé de nombreuses crêtes et bosses carbonacées dissimulant parfois presque entièrement 
l'épithécium brun-rouge; ascocarpes presque toujours

agglomérées. Lichen fréquent... . . . . (fig. 25) s. simplex

3. Disque des apothécies lisse, brun-rouge (surtout une fois mouillé), concave, pourvu dans de rares cas d'un "bouton" central et carbonacé; fructifications isolées ou groupées. Lichen fréquent. . . . . . . . . . . . . . . . s. privigna

\section{STAUROTHELE}

1. Lichen qui habite sur les roches périodiquement submergées ou mouillées; thalle brun foncé, lisse, fendillé-aréolé, pourvu de périthèces enfoncés dans celui-ci et formant des verrues proéminentes et bien visibles; 2 spores par asque, 27-34 x 16-17 $\mu \mathrm{m}$. Taxon commun; colonise les roches non calcaires ................. . . (fig. 26) s. fissa

1. Lichen qui vit sur les roches sèches; périthèces entièrement enfoncés dans le thalle... . . . . . . . . . . . . . 2 .

2. Spores au nombre de 2 par asque, 30-40 × 12-20 $\mu \mathrm{m}$; thalle brun, verruqueux à aréolé. Taxon fréquent; s'établit sur le calcaire... . . . . . . . .

2. Spores au nombre de 4-6 par asque, 25-28 x 10-12 $\mu \mathrm{m}$; thalle gris verdâtre pâle, lisse, fendillé-aréolé; médulle noire. Taxon fréquent; s'installe sur les roches siliceuses... . . . . . . . . s. diffractella

\section{VERRUCARIA}

1. Thalle blanc, extrêmement mince, endolithique, ou absent. . . . . 2 . 1. Couleur du thalle qui varie entre gris terne ou olive et à brun foncé ou noir, thalle épais ou plutôt mince; épilithique . . . . 3.

2. Périthèces entièrement enfoncés, formant des cratères distincts dans la roche; très petits périthèces de $0,15-0,16 \mathrm{~mm}$ de diamètre; involucrellum se fondant avec le rebord propre et ordinairement pas distinguable; spores 23-31 x 10,5-13,5 $\mu \mathrm{m}$. Lichen fréquent 
2. Périthèces presque entièrement enfouis, à certains

endroits du thalle, creusent parfois des périthèces dans

le substrat; fructifications habituellement semblables

à des bosses noires, de 0,25-0,4 mm de diamètre;

involucrellum d'ordinaire distinguable du rebord propre;

spores 18-26 × 9,3-11,5 $\mathrm{m}$. Lichen peu fréquent. . . $\underline{\mathrm{V}}$. muralis

3. Espèce qui pousse sur les rochers mouillés ou dans les cours

d'eau. Spores 25-30 x 10-12 $\mu \mathrm{m}$; involucrellum épais, noir,

qui se distingue du rebord propre mince, brun ou noir; thalle

mince, lisse à fendillé. Rare . . . . . . . . . $\underline{\text { V }}$ aethiobola

3. Espèces qui poussent sur les rochers secs; spores moins de

25 um de long. . . . . . . . . . . . . . . . . 4 .

4. Thalle aréolé et disjoint, brun ou brun grisâtre, épais;

spores 13-21 x 6-7,5 $\mu \mathrm{m}$; périthèces aux $3 / 4$ enfouis dans

le thalle, paroi noire, seulement au sommet

(involucrellum); rebord propre pâle; médulle noire

en-dessous, incolore sur le dessus. Rare... . . . . . $\underline{\text {. }}$ virens

4. Thalle uniforme et fendillé ou fendillé-aréolé, non

dispersé, mince ou épais. . . . . . . . . . . . . . . . 5 .

5. Couleur du thalle qui varie entre brun foncé et noir; très

épais, fendillé-aréolé; médulle noire . . . . . . . . . . . . . . 6.

5. Couleur du thalle qui varie du gris verdâtre au gris

brunâtre; médulle blanche (tirant légèrement sur le brun

terne) ou trop mince pour être distinguée. . . . . . . . . . . 7 .

6. Très petits périthèces, de 0,10-0,13 mm de diamètre, un

bon nombre d'entre eux se retrouvent sur chaque aréole,

émergeant partiellement du thalle; spores $16-17 \times 6-7$

um. Espèce peu fréquente. . . . . . . . . . . . $\underline{\text { V }}$ fuscella

6. Périthèces de 0,16-0,24 mm de diamètre, seuls ou en

petit nombre dans chaque aréole, entièrement ou à moitié

enfoncés seulement dans le thalle; spores $14-24 \times 7-11$

um. Espèce commune . . . . . . . . . . . . . ‥

7. Spores étroites, 14-25 x 4-6 $\mu \mathrm{m}$; petits périthèces, de

0,15-0,25 mm de diamētre, à moitié enfouis dans le thalle;

coloration thalline qui s'échelonne entre olive et gris

brunâtre; mince, granuleux à finement aréolé. Lichen rare 
7. Spores ellipsoïdes, $16-24 \times 8-10 \mu \mathrm{m}$; périthèces de 0,25-0,40 mm de diamètre, seulement au tiers enfouis; thalle gris terne.

Lichen peu fréquent. . . . . . . . . . . . . ‥ calkinsiana 


\section{LES LICHENS FOLIACÉES}

\section{Synopsis}

1. Thalle orange, jaune ou vert jaunâtre . . . . . . . . . . . . . . . 2.

1. Thalle gris, vert-de-gris, brun ou olive, dépourvu de toute trace de jaunâtre . . . . . . . . . . . . . . . . . . 16 .

2. Thalle orange ou jaune orange; face supérieure KoH+ pourpre foncé . . . . . . . . . . . . . . • . 3. (p.78)

2. Thalle jaune ou vert jaunâtre; face supérieure KoH- • • 5. (p. 78)

16. Thalle mouillé devient translucide et gélatineux; présence d'algues à toutes les profondeurs du thalle . . . . . . . 17. (p. 82)

16. Thalle mouillé ne devient jamais gélatineux; algues formant une couche algale bien définie . . . . . . . . . . . . . . . . . 37.

37. Une fois apparente, la couche algales (contient des algues bleues) est vert-bleu sombre; thalle à l'état sec vert brunâtre à brun . . . . . . . . . . . . . . . 38 .

37. Une fois apparente, la couche algale, (faite d'algues vertes) est vert pré . . . . . . . . . . . . . . . 54 .

38. Présence de sorédies ou d'isidies . . . . . . . . . . . . 39. (p. 87)

38. Absence de sorédies ou d'isidies . . . . . . . . . . . . 45. (p.88)

54. Plante qui croît en eau vive, sur des rochers ou sur les bords d'un cours d'eau sur des pierres immergées de temps en temps . . . . . . . . Dermatocarpon weberi (p. 91)

54. Lichen qui ne pousse pas sur des rochers immergés en tout temps ou de temps à autre . . . . . . . . . . . . 55.

55. Thalle ombiliqué; lichen saxicole . . . . . . . . . . . . . 56. (p. 91)

55. Thalle non ombiliqué . . . . . . . . . . . . . . . . 6l.

61. Thalle pourvu de très grandes fructifications (apothécies) enfoncées dans des dépressions; plante qui vit sur les mousses calcicoles et les sols sur calcaire. Taxon rare......... . . . . . Solorina saccata (p. 92) 
61. Lobes du thalle dépourvus d'apothécies enfoncées dans

des dépressions... . . . . . . . . . . . 62 .

62. Thalle gonflé et creux d'un bout à l'autre... . . . . 63. (p. 92)

62. Thalle plein (exception faite de l'extrémité des lobes d'un

petit nombre d'espèces) . . . . . . . . . . . . . 64 .

64. Thalle pourvu de sorédies ou d'isidies (pas de lobules

aplatis) . . . . . . . . . . . . . . 65.

64. Thalle dépourvu de sorédies ou d'isidies, mais quelques

espèces ont des lobules . . . . . . . . . . . . . . 95.

65. Lobes très larges dont la largeur varie ordinairement entre 8

et $30 \mathrm{~mm}$; thalle recouvert d'un réseau de creux et de crêtes

. . . . . . . . . . . . . . Lobaria pulmonaria (p.94)

65. Lobes de 0,5 à $8 \mathrm{~mm}$ de large. . . . . . . . . . . . . . 66 .

66. Thalle isidié, jamais sorédié . . . . . . . . 67. (p.94)

66. Thalle sorédié ou pourvu d'isidies grossièrement

granuleuses qui, par altération, se transforment en

sorédies avec l'âge . . . . . . . . . . . . 72 .

72. Sorédies situées surtout au pourtour des lobes ou à leur

extrémité . . . . . . . . . . . . . . . 73. (p.95)

72. Sorédies surtout réparties en plaques à la surface du thalle,

mais situées parfois à sa périphérie. . . . . . . . . . 89. (p. 99)

99. Lichens terricoles . . . . . . . . . 100. (p. 101)

99. Lichens saxicoles, lignicoles ou qui poussent sur

l'écorce . . . . . . . . . . . . . . . 102.

102. Thalle brun, brun olivâtre ou gris verdâtre sombre, mais

jamais gris clair; cortex supérieur $\mathrm{KOH}-$. . . . . . 103. (p. 103)

102. Thalle blanc grisâtre, gris clair ou nuancé de bleu; surface

supérieure KOH+ jaune . . . . . . . . . . 113. (p. 106) 


\section{Clef Générale}

(N.B.: Les espẽces squamuleuses sont considérées comme des lichens "crustacés", v. p. 16 et p. 30).

1. Thalle orange, jaune ou vert jaunâtre . . . . . . . . . . . . . . 2 .

1. Thalle gris, vert-de-gris, brun ou olive, dépourvu de toute trace de jaunâtre . . . . . . . . . . . . . . . . . . 16 .

2. Thalle orange ou jaune orangé; face supérieure KOH+ pourpre . . . . . . . . . . . . . . . . . . . . . . 3.

2. Thalle jaune ou vert jaunâtre; face supérieure koH- . . . . . . 5 .

3. A l'extrémité des lobes du thalle, le revers est recouvert de sorédies, les lobes étant souvent en forme de capuchon; absence d'apothécies. Plante commune sur les arbres en bordure des routes, surtout sur les ormes, et quelquefois sur le roc.. . . . . . . . . . . . (fig. 31) Xanthoria fallax

3. Absence totale de sorédies; lobes étroits et souvent finement

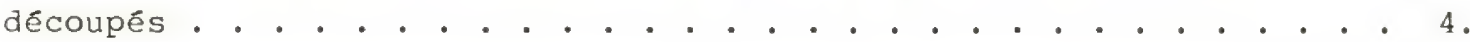

4. Lichen corticole. Apothécies pourvues de coupes orange presque toujours présentes et habituellement abondantes; lobes étroits et épais. Plante assez commune, poussant particulièrement sur les peupliers et les trembles

4. Lichen saxicole, ordinairement calcicole, ou rencontré sur les rochers fréquentës par les oiseaux (nids ou perchoirs). Lobes étroits, souvent allongés; thalle intimement lié au roc et presque crustacé; plante rarement pourvue d'apothécies dans la région d'ottawa

\section{Xanthoria elegans}

5. A l'état sec, thalle citrin vif à couleur jaune d'oeuf . . . . . 6.

5. Thalle présentant des tons de vert jaunâtre ou de jaune verdâtre . . . . . . . . . . . . . . . . . . . . . . . . 8 . .

6. Médulle citrin vif; lobes ronds, de $l$ à $3 \mathrm{~mm}$ de large; sorédies disposées à la périphérie des lobes en bandes jaunes et farineuses. Lichen colonisant le bois, l'écorce ou les rameaux de conifères et de bouleaux, mais rarement les roches, et frëquemment retrouvé dans les tourbières . . . . . . . . . . . . Cetraria pinastri 

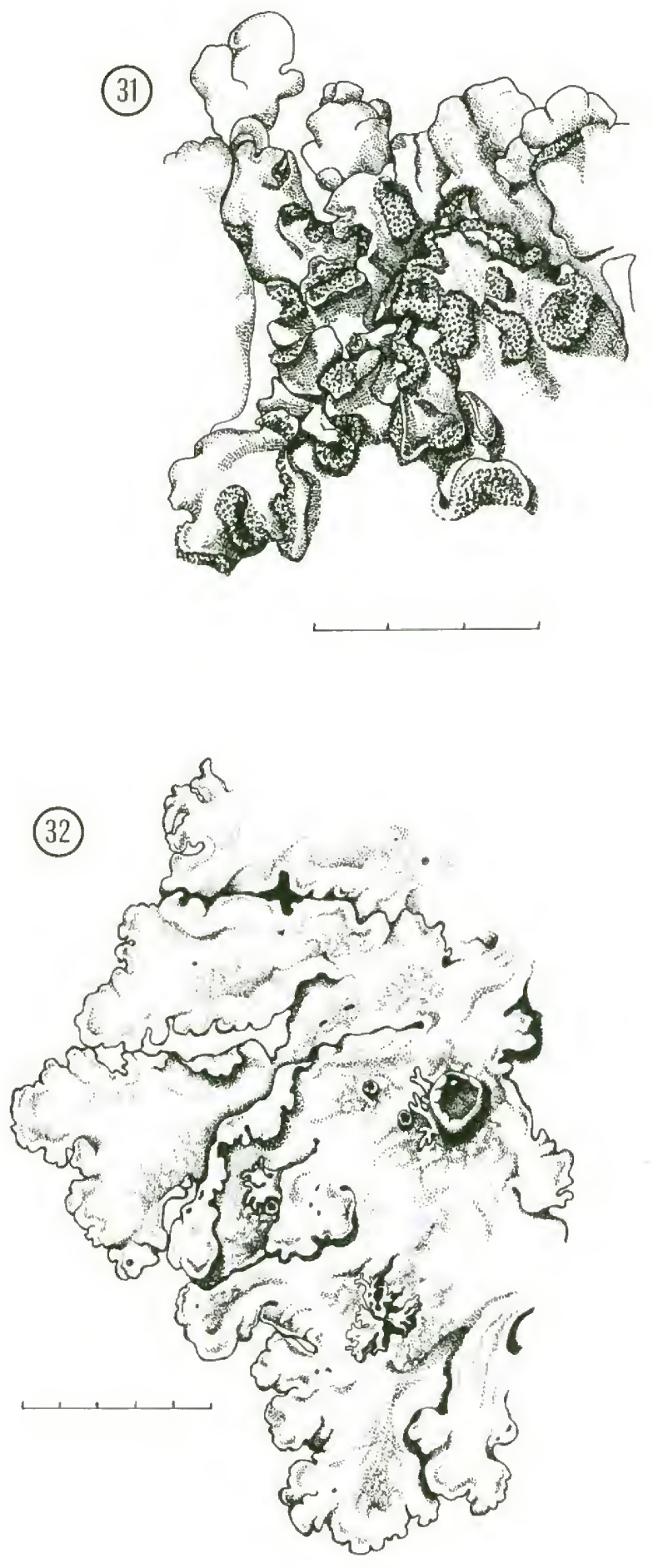

Figures 31 et 32.-31. Xanthoria fallax.-32. Parmelia cumberlandia. Échelle: chaque division $=1 \mathrm{~mm}$. 
6. Médulle blanche; lobes finement découpés (moins de I mm de large) . . . . . . . . . . . . . . . . . . 7 .

7. Lobes souvent pourvus de structures granulaires jaunes, ou sorédies, tout le long de leur marge; thalle se réduisant de temps en temps à une croûte granuleuse jaune. Taxon commun sur les arbres en bordure des routes, sur l'orme, le frêne, etc. . . . . . . . . . . . . . . . . . Candelaria concolor

7. Pas de sorédies ou de granules sur les lobes. Lichen rare . . . . . . . . . . . . . . . . . . . Candelaria fibrosa

8. Médulle KOH+ jaune puis rouge; absence de sorédies.

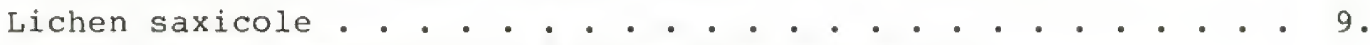

8. Médulle KоH-; présence de différentes sortes de sorédies. Lichen s'installant sur l'écorce ou le bois, mais rarement sur le roc . . . . . . . . . . . . . 12.

9. Thalle couvert d'isidies . . . . . . . . . . . . . . . . . 10.

9. Thalle dépourvu d'isidie en surface . . . . . . . . . . . . . . 11 .

10. Dessous du thalle noir de jais, sauf le contour qui est

brun foncé. Lichen peu fréquent. . . . . . Parmelia conspersa

10. Tout le revers du thalle brun pâle à brun franc. Plante fréquente . . . . . . . . . . . . . Parmelia plittii

11. Medulle $\mathrm{KOH}+$ jaune puis rouge sang Pat jaune vif ou orange (acide salazinique); thalle adhérant lâchement au roc et croissant souvent sur lui-même; lobes généralement étroits, de 1 à $3 \mathrm{~mm}$ de large. Plante commune . . . . . . Parmelia taractica

11. Médulle KoH+ jaune (parfois rouge), Pat orange ou orange rougeâtre (acides stictique et norstictique); en règle générale, thalle intimement lié au roc et formant un plant rond à un seul feuillet; lobes souvent plus larges que $3 \mathrm{~mm}$. plante commune . . . . . . . . (fig. 32) Parmelia cumberlandia

12. Lobes découpés, très étroits, de 0,5 à $1,5 \mathrm{~mm}$ de large, appliqués et fermement fixés au support; soralies disposées en grosses masses farineuses globuleuses; médulle C-, KOH- et $\mathrm{Pd}-$. Lichen relativement commun sur le bois ou l'écorce des conifẽres . . . . Parmeliopsis ambigua 
12. Lobes dépassant 1,5 mm de largeur, non découpés finement

13. Sorédies disposées en masses verruculeuses et irrégulières à la surface du thalle, jamais à la marge; très gros lobes dont la largeur atteint et dépasse parfois $6 \mathrm{~mm}$, et qui forment des thalles ronds . . . . . . . . . . . . . . . . . . . 14 .

13. Sorédies marginales, au moins en partie; lobes de 2 à $4 \mathrm{~mm}$ de large; médulle $\mathrm{Pd}$ - . . . . . . . . . . . . . . . . . 15 .

14. Lobes couverts de taches blanches, apparentes, rondes ou irrégulières (pseudo-cyphelles) sur la face supérieure; médulle C+ rouge, $\mathrm{Pd}-, \mathrm{KC}+$ rouge (acide lécanorique). Rare; corticole. . . . . . . . . . . Parmelia flaventior

14. Lobes dépourvus de taches blanches sur la face supérieure; médulle C-, Pd+ rouge orangé, $\mathrm{KC}+$ rose (acide protocétrarique). Très commun; pousse surtout sur l'écorce et, parfois, sur la roche . . . Parmelia caperata (N.B.: Une espèce très voisine, grossièrement pustuleuse à presque isidiée, $P$. baltimorensis, pousse sur la roche dans 1 'est des État-Unis et dans le sud de l'Ontario. Elle vit probablement dans l'outaouais.)

15. Thalle pourvu de rhizines sur le revers; pourtour du lobe rendu pulvérulent par les sorédies; lobes élancés; médulle C-. Plante poussant sur l'écorce (normalement de résineux) et le vieux bois; taxon commun . . . . . . . . . Cetraria oakesiana

15. Beaucoup de rhizines sur le revers; sorédies dispersées à la surface des lobes (superficielles) aussi bien qu'en périphêrie; lobes arrondis non allongês; médulle c+ vermeille. Lichen rare; pousse sur l'écorce. • Parmelia ulophyllodes

16. Une fois mouillé, thalle devenant translucide et gélatineux, vert brunâtre foncé ou vert bleuâtre; à l'état sec, sa couleur varie de gris brunâtre à gris ardoise ou noir; présence d'algues à toutes les profondeurs du thalle (fig. 33, 35) . . . . . . . . . . . . . 17.

16. Une fois mouillé, thalle jamais gélatineux; algues ne se trouvant que dans une couche algale bien définie (fig. 65) 37. 
17. Face inférieure recouverte de longs poils fins et blancs (tomentum); thalle gris, isidié. Plante rare

. . . . . . . . . . . . . . Leptogium burnetiae var. hirsutum

17. Face inferieure lisse, dépourvue de poils ou tomentum . . . . . . 18.

18. Thalle gris acier; lobes minces de 1 ou 2 à $8 \mathrm{~mm}$ de

large; faces supérieure et inférieure du thalle

recouvertes d'un cortex (visible sur une prēparation microscopique) (fig. 35) . . . . . . . . . . . . . . 19.

18. Thalle présentant des tons de brun, d'olive, de vert ou de noir (exceptionnellement de gris chez Leptogium Zichenoides); thalle pourvu ou dépourvu de cortex . . . . . . . 22.

19. Surface du thalle (et parfois son contour) couvert d'isidies dont la forme varie de cylindrique à aplatie . . . . . . . . . . . 20 .

19. Thalle dépourvu d'isidies. Lichen rare . . . . . . . . . . . . 21.

20. Apothécies plates et abondantes; lobes gris foncé à olive, de 1 à $4 \mathrm{~mm}$ de large. Lichen rare; colonise les rochers calcaires . . . . . . . . . . Leptogium dactylinum

20. Apothécies rares ou absentes; lobes minces, gris acier. Lichen commun; au pied des arbres et sur les rochers moussus . . . . . . . . . (fig. 36) Leptogium cyanescens

21. Largeur des lobes variant entre 1,2 et 2,4 mm, lobes accompagnés de temps en temps de petits lobules de régénération; grand nombre d'apothécies, de 0,3 à $0,7 \mathrm{~mm}$ de large. Lichen se trouvant à la base des arbres, sur les

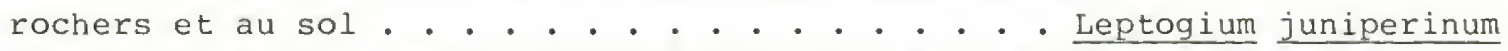

21. Largeur des lobes très minces variant entre 2 et 8 mm; dans certains cas, apothécies abondantes, de 0,5 à $2 \mathrm{~mm}$ de large. Lichen vivant sur l'écorce (N.B.: comme L. cyanescens, mais dépourvu d'isidies) . . . . . . . . . . . Leptogium azureum

22. Thalle uniformément coloré, variant de brun à brun rougeâtre (exceptionnellement gris); lobes de 1 à $3 \mathrm{~mm}$ de large, plus ou moins dressês, bordẻs de protubërances dentellées, finement découpées et semblables à des isidies (fimbriées); présence de cortex supérieur et inférieur (fig. 35). Espèce croissant sur les rochers calcaires, l'écorce et habituellement enfouie partiellement dans la mousse; lichen frēquent . . . . . . . . . ( . . (fig. 37) Leptogium lichenoides 
(33)
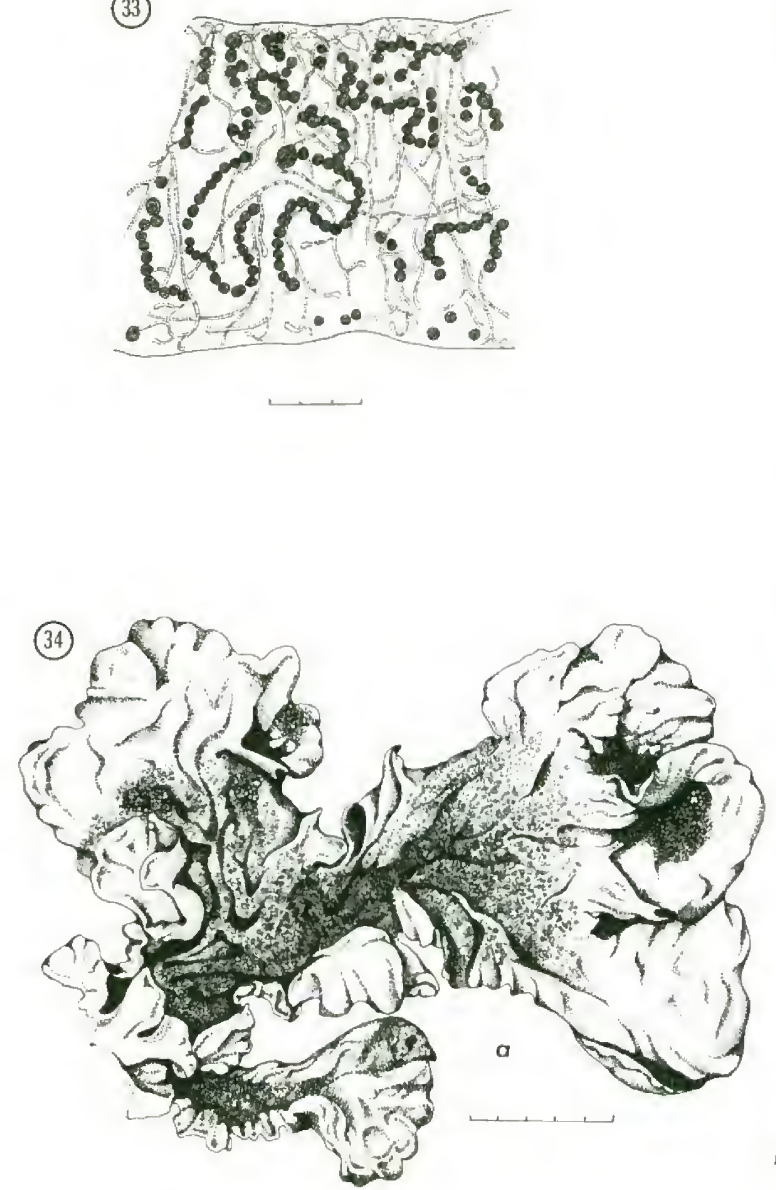

(35)
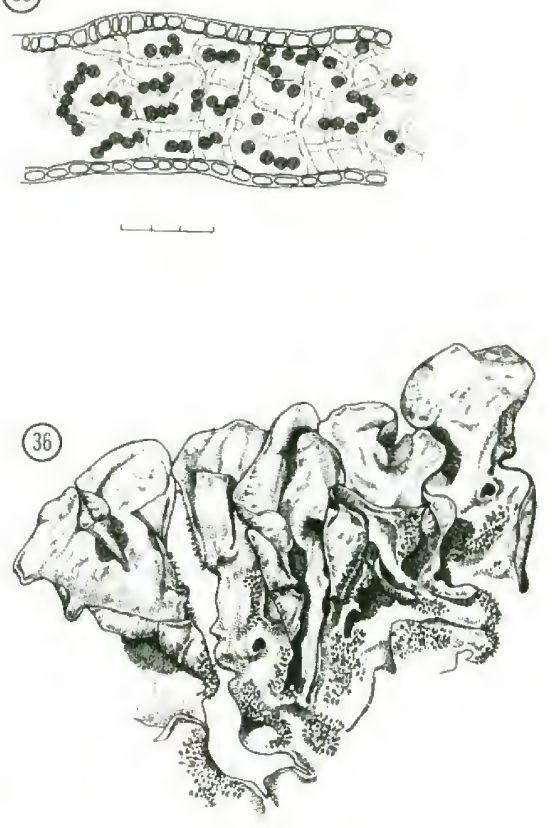

b

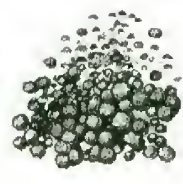

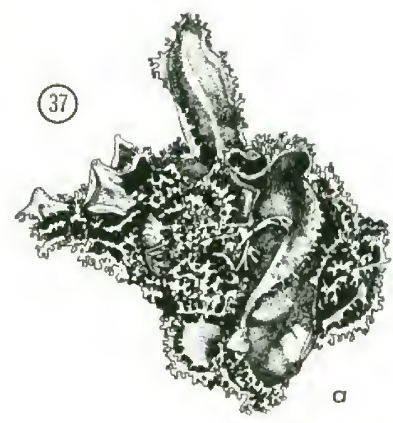

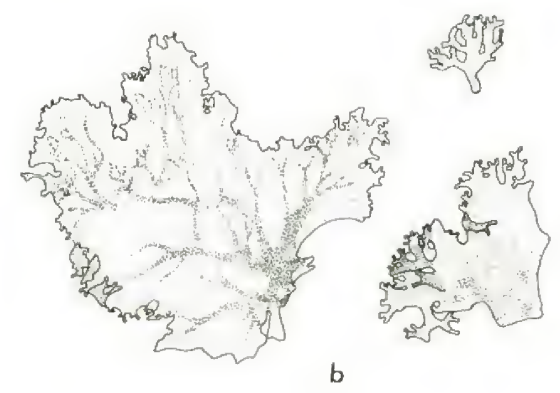

Figures 33 à 37.-33. Collema: coupe verticale du thalle.-34. Collema subflaccidum: a) thalle; b) isidies granuleuses.-35. Leptogium: coupe verticale du thalle. -36. Leptogium cyanescens.-37.

Leptogium lichenoides: a) thalle sec; b) lobes humides et aplatis.

Échelle:-33, 35: chaque division $=10 \mu \mathrm{m} ;-34,36,37$ : chaque division $=$ $1 \mathrm{~mm}$. 
22. Couleur du thalle variant entre noir olivâtre et noir verdatre; parfois coloration partiellement brunâtre et, dans ce cas, lobes épais et non fimbriés; thalle non cortiqué (fig. 33) . . . . . . . . . . . . . . 23 .

23. Lobes épais, spatulés, dressés, de 7 à $10 \mathrm{~mm}$ de haut, quelque peu découpés, dont les lobes terminaux mesurent de 0,7 à 1,5 mm de large, et dont la surface est souvent granuleuse ou couverte de minuscules granules (isidies); thalle noir; Gloeocapsa est le phycosymbionte. Espèce rare; pousse sur le calcaire. . . . . . . . . . . . . . Thyrea pulvinata

23. Lobes plus ou moins plats, non ascendants; thalle au moins en partie verdâtre ou olive; Nobtoc est le phycosymbionte . . . . . . 24.

24. Espèces qui vivent sur l'écorce. Lobes très importants de 5 à $15 \mathrm{~mm}$ de large . . . . . . . . . . . . . . . 25.

24. Espèces qui vivent sur les rochers et les sols calcaires. Lichen foliacé pourvu de lobes généralement de 0,5 à $4 \mathrm{~mm}$ de large; ou encore plante subcrustacée . . . . 26.

25. Lobes plus ou moins lisses, tout au plus légèrement plissés; thalle couvert d'isidies en forme de minuscules granules. Espèce très commune... . . . . . (f ig. 34) Collema subflaccidum

25. Lobes striés, plissés et pustuleux sur les faces supérieure et inférieure; isidies cylindriques, du moins en partie. Lichen peu commun . . . . . . . . . . Collema furfuraceum 26. Thalle stérile, dépourvu d'apothécies . . . . . . . . . . 27 .

26. Thalle fertile; apothécies pourvues d'ouvertures béantes ou en forme de points. . . . . . . . . . . . . . 29.

27. Thalle nettement brunâtre à brun rougeâtre, pourvu d'isidies granuleuses à sphériques; lobes très épais, dont la largeur va jusqu'à $4 \mathrm{~mm}$. Plante rare; colonise le calcaire.. . Collema sp.

27. Thalle olive à noirâtre . . . . . . . . . . . . . . . 28.

28. Lobes pourvus de marges visiblement épaisses ou boursouflées, souvent pliés longtidudinalement; isidies non remarquables ou absentes; lobes habituellement étendus à plat sur le support, se rétrécissant parfois 
et devenant plus ou moins ascendants. Lichen peu

fréquent; croît sur les sols et les mousses couvrant le calcaire. . . . . . . . . . . . . . . . . Collema tenax

28. Lobes plus ou moins minces, ni boursouflés ni pliés dans

le sens de la longueur, ordinairement dressés, découpés,

retroussés (comme des "Corn Flakes"), plus rarement

étendus à plat sur le substrat; thalle couvert de

grandes isidies globuleuses. Espèce fréquente; pousse

habituellement directement sur le calcaire . . Collema tuniforme

29. Spores entièrement ou presque entièrement non cloisonnées;

thalle subcrustacé, généralement membraneux; apothêcies

grêles (moins de $0,5 \mathrm{~mm}$ de large) . . . . . . . . . . . . . . . 30 .

29. Spores bicellulaires ou pluricellulaires à murales; thalle nettement lobé ou foliacé . . . . . . . . . . . . . . . . . . 31 .

30. Apothécies s'ouvrant par un petit ostiole et ressemblant

à des périthèces; spores hyalines, non-cloisonnées, à

membrane épaisse, subsphériques à elliptiques. Espēce

rare; muscicole et terricole.. . . . Lempholemma myriococcum

30. Apothécies discoïdes, à large orifice; spores parfois

uniseptées, à paroi mince, elliptiques à presque

fusiformes, 22-26 × 6-8 $\mathrm{m}$. Lichen rare; terricole et

muscicole. . . . . . . . . . . . cf. Lempholemma sp.

31. Spores uniseptées, au nombre de 8 par asque; lobes souvent

imbriqués, boursouflés au pourtour, dont les lobules gonflés

ressemblent parfois à des granules sphériques; apothécies

abondantes dotées de grands disques brun-rouge et de marges

épaisses. Taxon peu commun... . . . . Collema cf. coccophorum

31. Spores à 3 ou 4 cloisons ou encore murales.... . . . . . . . 32.

32. Une fois à maturité, les spores prêsentent 3 ou 4

cloisons...................... 33.

32. A maturité, spores surtout murales ou submurales..... . 34 .

33. Lobes pas ou à peine boursouflés au pourtour, munis ou non

d'isidies globuleuses ou granules; lobes devenant découpés,

dressés, très fertiles et pourvus de fines apothécies

aplanies, souvent portées sur de courts lobes latéraux ou 
marginaux; marges des apothécies minces et lisses. Lichen peu fréquent . . . . . . . . . . . . . . . . collema polycarpon

33. Lobes ascendants, légèrement découpés et nettement boursouflés au pourtour; absence d'isidies ou de granules; apothêcies rares, larges, fréquemment convexes, enfoncées dans le thalle ou encore fermement fixées à ce dernier. Lichen peu fréquent. . . . . . . . . . . . . . . Collema tenax

34. Lobes découpés, non boursouflés au pourtour; thalle souvent doté de lobes plus épais, affectant la forme de "Corn Flakes", recouvert irrégulièrement d'isidies globuleuses; apothécies peu abondantes; 8 spores par asque. Lichen commun, colonisant le calcaire. . Collema tuniforme

34. Lobes appliqués et fermement fixês au support, nettement boursouflés au pourtour, les plus vieilles parties devenant souvent un peu membraneuses; apothécies fréquentes, munies de grands aisques . . . . . . . . . . . 35 .

35. Thalle membraneux à évanescent et remplacé par des lobes gonflés et éparpillés; grosses apothécies abondantes, aux rebords habituellement minces et plans; spores submurales à murales, au nombre de 4 par asque, 25-34 x 10-13 $\mu \mathrm{m}$. Plante se trouvant particulièrement sur les sols et les mousses sur calcaire . . . . . . . . . . . . . . . . . . Collema limosum

35. Majeure partie de la périphérie thalline distinctement lobée, quoique parfois les vieilles divisions deviennent membraneuses; spores submurales, au nombre de 8 par asque . • • • 36 .

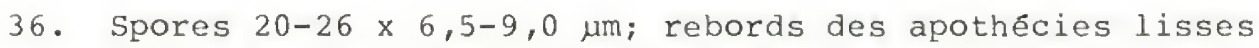
et aplatis; apothécies habituellement clairsemées. Lichen peu fréquent; terricole et parfois rupicole. . Collema tenax 36. Spores 25-34 × 9,0-13 um; marges des apothécies épaisses, dentelées à lobées; apothécies abondantes. Taxon fréquemment rencontré sur le calcaire ou les sols sur calcaire. . . . . . . . . . . Collema bachmanianum

37. Une fois apparente, la couche algale est vert-bleu foncé; lobes dont la largeur est de $5 \mathrm{~mm}$ ou plus, à l'exception du genre Pannaria, dont les espèces sont squamuleuses. Lichen poussant surtout sur la terre ou sur les tronçons 
d'arbre et les souches moussues, tout autant que sur les rochers ombragés. . . . . . . . . . . . . . . . . . . . . 38 .

37. Une fois apparente, la couche algale est vert pré; lobes de $0,3 \mathrm{~mm}$ de large, jamais granuleux . . . . . . . . . . . . 54 .

38. Présence de sorédies ou d'isidies . . . . . . . . . . 39.

38. Absence de sorédies ou d'isidies. . . . . . . . . . . 45 .

39. Sorédies ou isidies surtout marginales . . . . . . . . . . . 4 40.

39. Sorédies ou isidies superficielles, non marginales; face inférieure de blanche à noire, feutrée (dêpourvue de cortex) . . . . 43.

40. Face inférieure du thalle feutrée (dépourvue de cortex) . . . 4l. 40. Face supérieure du thalle lisse, cortiquée et teintée

d'ocre. Espèce rare; lichen de forêts denses, poussant sur les rochers, les troncs et, de temps à autre, sur l'ecorce . . . . . . . . . . . . . Nephroma parile

41. Contour du thalle garni d'abondantes sorédies granuleuses, d'une coloration grise nuancée de bleu; thalle subsquamuleux à foliacé. Taxon trēs rare; rencontré au pied d'arbres moussus . . . . . . . . . . . . . . . Pannaria ahlneri

41. Contour du thalle avec des "isidies" planes, lobulées et pas de sorédies; thalle foliacé dans sa majeure partie. Lichen terricole. . . . . . . . . . . . . . . . 4 42 .

42. Face inférieure noire, maculée de quelques taches blanches, surtout au bord; face supérieure luisante ou parfois, scabre à pruineuse, du moins en périphérie. Plante peu fréquente......... Peltigera elizabethae

42. Face inférieure pâle, pourvu d'un réseau de veines saillantes; face supérieure mate et tomenteuse près de la périphérie. Espèce rare.... . . Peltigera praetextata

43. Thalle de petite taille, doté de lobes dont la largeur ne dépasse pas 5 à $8 \mathrm{~mm}$. . . . . . . . . . . . . . . . 44 44

43. Très grand thalle aux larges lobes (largeur excédant couramment $15 \mathrm{~mm})$, aplatis, munis d'un revêtement fait de minuscules isidies ou granules fixées lâchement et empruntant des formes variées allant de cylindriques à presque globuleuses. Taxon terricole dans les stations ombragées . . . . . . . . . . . . . . . . Peltigera evansiana 
44. Thalle pourvu de plaques de sorédies rondes, individualisees et gris clair; lobes fortement concaves.

Espèce rare; terricole... . . . . . . . Peltigera spuria

44. Thalle doté de menues isidies brunes et squamiformes disséminées sur la face supêrieure; absence de sorêdies; en règle générale, lobes plans. Lichen rare; terricole, croît en milieu ouvert........ . Peltigera lepidophora

45. Thalle composé presque entièrement de lobes squamiformes, se chevauchant plus ou moins. Plante peu fréquente; rupicole, crô̂t dans les milieux ombragés.. . . . . . Pannaria leucophaea

45. Thalle nettement foliacé . . . . . . . . . . . . . . 46.

46. Face inférieure cortiquée, de couleur brun jaunâtre clair ou chamois, pourvue ou non de tomentum; lobes de 2 à $6 \mathrm{~mm}$ de large; thalle généralement fertile, ayant de grandes fructifications discoïdes et rousses situées au revers de l'extrémité des lobes . . . . . . . . . 47.

46. Face inférieure dépourvue de cortex, (feutrée); lobes de 4 à $15 \mathrm{~mm}$ de large; si le lichen est fertile, présence de fructifications sur le dessus de l'extrémité de lobes spéciaux . . . . . . . . . . . . . . . . 48 .

47. Revers lisse, ni tomenteux, ni verruqueux; lobes de 2 à $4 \mathrm{~mm}$ de large, se chevauchant souvent. Espèce peu fréquente .. . . . . . . . . . . . . . . . . Nephroma bellum

47. Revers recouvert d'un tomentum court et blanc de même que de verrues éparpillées; lobes de 3 à $6 \mathrm{~mm}$ de large. Taxon rare

. . . . . . . . . . . . . . . . . Nephroma resupinatum

48. Revers du thalle surtout brun, parsemé de plaques

blanches, ce caractère ne s'observant parfois que sur

les plus jeunes sections du lobe; veines ou crêtes filiformes non évidentes . . . . . . . . . . . . 49.

48. Revers du thalle surtout blanc, couvert de veines individualisées blanches ou brunes, ou crêtes filiformes .. . . . . . . . . . . . . 52.

49. Dessous parsemé de quelques taches blanches, concentrées pour la plupart au bord des lobes; marges et fentes du thalle 
souvent pourvues de petits lobules ou squamules; apothécies rondes, ne sont pas portées sur des lobes pédicellés; spores à 3 cloisons, moins de $45 \mu \mathrm{m}$ de long. Peu fréquent

. . . . . . . . . . . . . . . . Peltigera elizabethae

49. Dessous parsemé de taches blanches, apparentes, disposées entre un réseau de sections plates et brunes, qui se fondent ensemble au milieu du thalle; thalle dépourvu de lobules ou squamules . . . . . . . . . . . . . . . . . . . . 50 .

50. Spores fusiformes, montrant 3 ou 4 cloisons; apothécies presque arrondies, brun rougeâtre foncé, aplaties, ni dresées, ni révolutées. Espèce rare. . . Peltigera horizontalis

50. Spores en forme d'aiguille (aciculaires), 3-9 cloisons, longueur plus de $45 \mu \mathrm{m}$; apothécies allongées, dressées, en forme de selle . . . . . . . . . . . . . 51.

51. Largeur des lobes 6-12 mm surtout; apothécies 2-4 mm de longueur, noires, situées au bord des lobes ou sur des lobes trẽs courts; extrémités des lobes deviennent souvent légèrement pruineuses. Fréquent; pousse dans les forêts fermées . . . . . . . . . . . . . . . . . . Peltigera neckeri

51. Largeur des lobes 10-22 $\mathrm{mm} ;$ apothécies $3-6 \mathrm{~mm}$ de long, brun-rouge foncé, situées habituellement au bout de lobes en forme de doigt; lobes toujours dépourvus de pruine. Rare ... . . . . . . . . . . . Peltigera polydactyla $\underline{\text { s. }} \underline{\text { str. }}$

52. Lobes couverts d'un duvet clairsemé ou tomentum à la surface, particulièrement près de l'extrémité des lobes . . . . . . . . . . . . . . . . . . . . . 53.

52. Surface du thalle très mate, non tomenteuse, rugeuse ou croûteuse (scabrée); lobes généralement larges et arrondis. Lichen peu fréquent... . . . . . Peltigera scabrosa

53. A l'état sec, lobes à marge épaissie et recourbée. Espèce commune; terricole, recherche les endroits secs ................... (... (fig. 38) peltigera rufescens

53. Marge des lobes ni épaissie, ni visiblement recourbée. Espèce commune; s'installe à l'ombre ou dans les milieux humides, sur la mousse, les tronçons d'arbre ou l'humus . . . Peltigera canina 

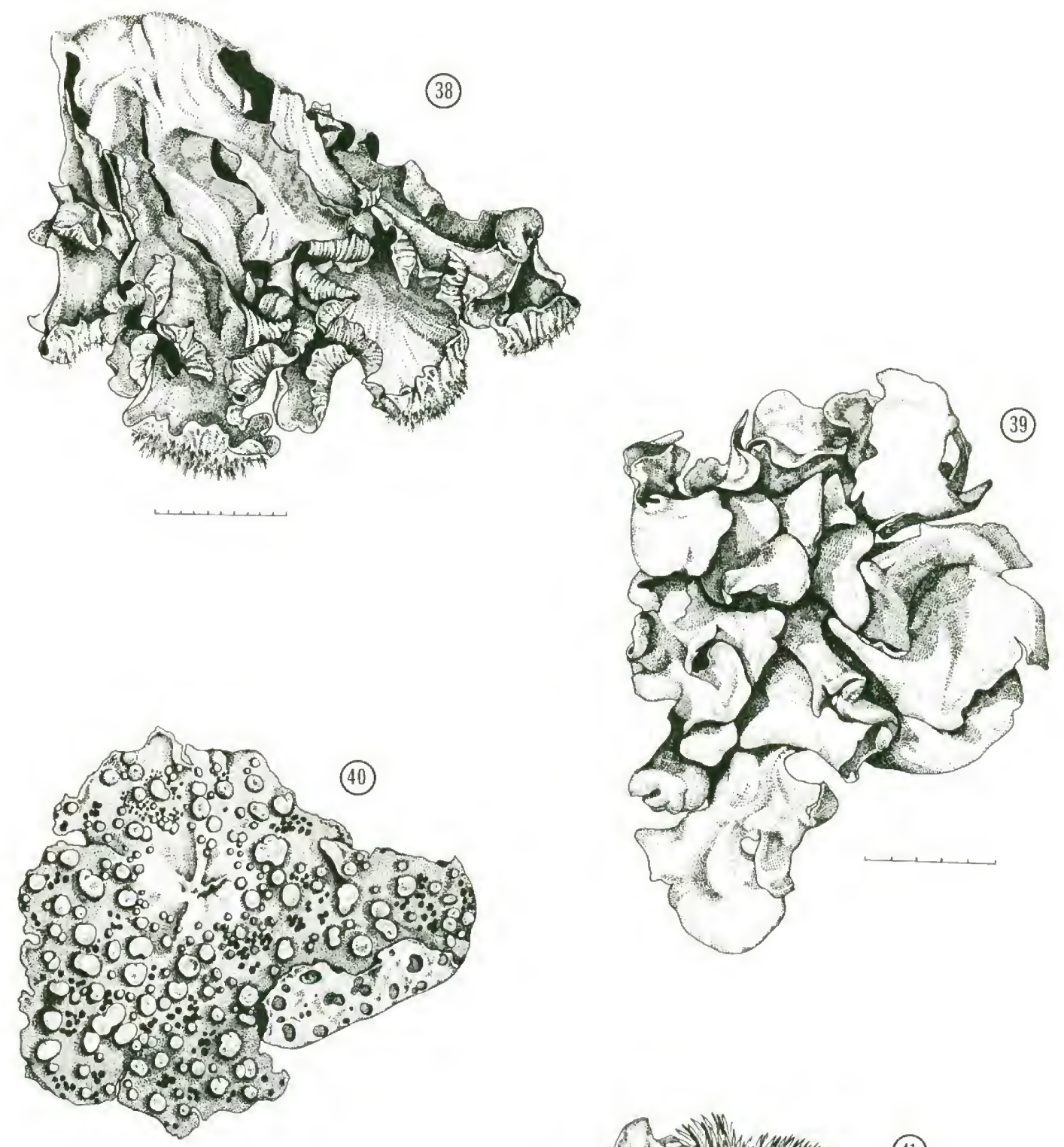

Figures 38 à 41.-38. Peltigera rufescens. -39. Dermatocarpon weberi. -40. Lasallia papulosa. -41. Solorina saccata. Échelle: chaque division $=1 \mathrm{~mm}$. 
54. Plante croissant en eau vive sur des rochers ou au bord d'un cours d'eau, sur des pierres immergées de temps en temps; une fois mouillé, thalle verdâtre, à l'état sec brun; appareil végétatif composé de petits lobes entassês; revers lisse ou bien parfois strié. Lichen commun . . . . . . . . (fig. 39) Dermatocarpon weberi

54. Lichen ne poussant pas sur des rochers immergés en tout temps ou de temps à autre . . . . . . . . . . . . 55.

55. Thalle plus ou moins rond, non lobé, fixé au substrat par un seul point central (ombiliqué); coloration brune à gris brunâtre. Lichen saxicole. . . . . . . . . . . . . 56.

55. Thalle adhérant au support par une portion importante de sa face inférieure ou pourvu de rameaux ténus et ascendants formant des coussinets presque fruticuleux . . . . . . . . . .61.

56. Thalle couvert de grosses pustules, saillantes au-dessus et creuses au-dessous; revers lisse (pas de rhizines). Lichen peu fréquent........ (fig. 40) Lasallia papulosa 56. Face superrieure du thalle plus ou moins lisse, dépourvue de pustules... . . . . . . . . . . . . 57.

57. Face supérieure couverte d'isidies finement granuleuses; thalle friable, extrêmement mince et presque noir. Taxon peu fréquent............... . . . Umbilicaria deusta

57. Face supérieure non granuleuse ou isidiée . . . . . . . . . . 58.

58. Face inférieure brun jaunâtre clair, parfaitement lisse, dépourvue de rhizines ou de lamelles de tissu; thalle gris à brun jaunâtre clair, parsemé de minuscules points noirs indiquant la présence de périthèces enfouis. Plante rare; colonise en général différentes sortes de calcaire. . . . . . . . . . . Dermatocarpon miniatum

58. Face inférieure jamais lisse, présentant un coussin de rhizines ou de lamelles de tissu; les points noirs à la surface, quand il y en a, sont dus à des pycnides, pas à des périthèces. Espèce croissant sur la "roche dure", par ex., le granite. . . . . . . . . . . . . . 59.

59. Revers composé de tissus imbriqués, réticulês en forme de lamelles rayonnant autour du point de fixation; rhizines 
absentes ou très clairsemées; fructifications noires (apothécies) pourvues de crêtes irrêgulières et de surfaces ridées habituellement apparentes. Espèce commune

59. Revers presque entièrement recouvert de rhizines noires

formant un revêtement mince quoique dense et aussi pourvu de lamelles de tissu; pas d'apothécies. . . . . . . . . . . 60 .

60. Thalle très épais, gris pâle à brun grisâtre pâle et rigide (un peu comme du carton tout en étant fragile). Lichen rare............ . . Umbilicaria vellea 60. Thalle relativement mince, présentant une teinte uniforme de brun à brun foncé. Lichen commun

- Umbilicaria mammulata

61. Lobes possédant des apothécies en forme de grands disques bruns (jusqu'à $4 \mathrm{~mm}$ de diamètre) enfoncées dans des dépressions. Taxon rare; vit sur les mousses calcicoles ou les sols sur calcaire.......... (fig. 4l) Solorina saccata

61. Lobes dépourvus d'apothécies enfoncées dans des dépressions .. . . . . . . . . . . . . . . 62

62. Thalle boursoufflé et creux; revers noir, tout à fait dêpourvu de rhizines; pas de cils marginaux . . . . . . . 63.

62. Thalle plein (si l'extrémité des lobes est renflée, le revers est alors blanc et on remarque la présence de longs cils marginaux)................ 64 .

63. Éclatement de l'extrểmité des lobes, ce qui donne une structure en forme de capuchon regorgeant de sorédies granuleuses. Lichen très fréquemment rencontré sur l'écorce, les rameaux et le bois........ (fig. 42) Hypogymnia physodes

63. Si elles sont présentes, sorédies réparties en plaques à la surface; face supérieure perforée. Taxon rare; recherchant l'écorce . . . . . . . . . . . . . Menegazzia terebrata

64. Thalle granuleux ou pulvérulent (sorédies), ou bien pourvu d'isidies (et non de lobules). . . . . . . . . . 65 .

64. Thalle ni sorédié, ni isidié, mais pourvu de temps à autre de lobules. . . . . . . . . . . . . . 99. 

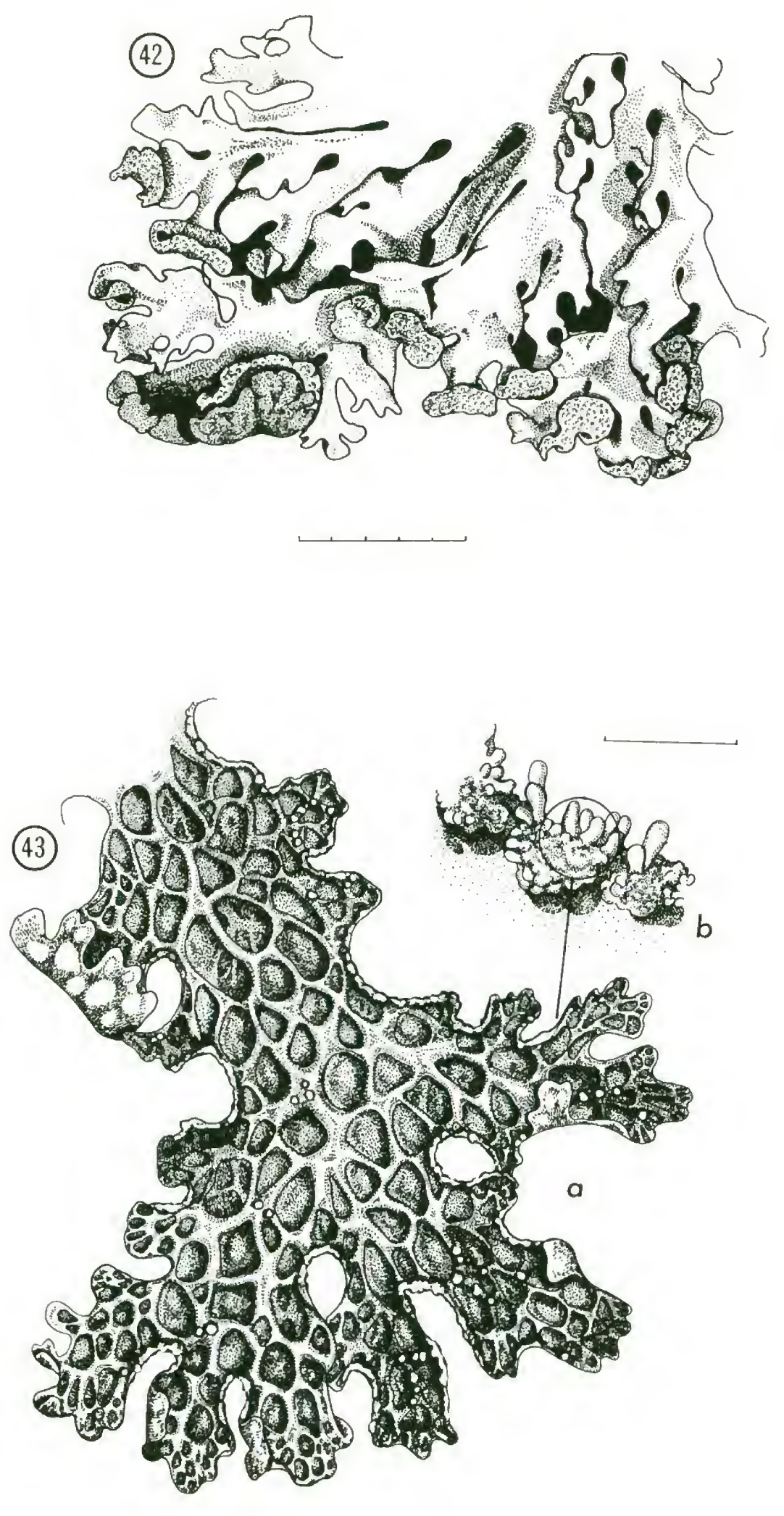

Figures 42 et 43.-42. Hypogymnia physodes. -43. Lobaria pulmonaria:

a) thalle; b) soralies isidiēes. Échelle: chaque division $=1 \mathrm{~mm}$. 
65. Très grands lobes dont la largeur varie entre 8 et $30 \mathrm{~mm}$; thalle couvert d'un réseau de creux et de crêtes, pourvu de sorédies ou d'isidies ou des deux se retrouvant seulement sur ces veines; face inférieure brun jaunâtre pâle, couverte d'un tomentum duveteux et dépourvue de vraies rhizines. Lichen rare; vit sur les arbres, ordinairement dans les forêts denses et riches . . . . . . . . . (fig. 43) Lobaria pulmonaria

65. Lobes de 0,5-6 mm de large, ou s'ils sont plus larges, dépourvus d'un réseau de crêtes et de creux . . . . . . . . . . . 66 .

66. Thalle toujours dépourvu de sorédies et pourvu d'isidies plus ou moins cylindriques, quelquefois rameuses . . . . . . 67.

66. Thalle pourvu de sorédies ou d'isidies verruciformes s'altérant avec l'âge et se métamorphosant en sorédies . . . . 72.

67. A l'état sec, thalle brun ou brun olivâtre, isidies plus ou moins boursoufflées, spatulées ou claviformes; médulle C-, KOH- et Pd- . . . . . . . . . . . . . . Parmelia exasperatula

67. Thalle gris ou vert tirant sur le gris, mais jamais brun olivâtre . . . . . . . . . . . . . . . . . 68.

68. Minuscules points blancs (pseudocyphelles) disséminés sur la face supérieure des lobes thallins; coloration du revers brun jaunâtre pâle; médulle c+ rouge, KOH-, Pdi-. Taxon très commun; épiphyte ou lignicole, parfois saxicole... . . . . . . . . . . . . Parmelia rudecta

68. Thalle dépourvu de points blancs visibles; médulle C-, KOH+ jaune et Pdt jaune ou orange . . . . . . . . . . . . 69.

69. Petits lobes dont la largeur n'excède pas $1,5 \mathrm{~mm}$; revers luisant brun pâle; petit thalle rond, croissant souvent sur l'écorce ou le bois de conifères secs et durs; cortex et médulle KoH+ jaune vif, Pdt orange. Lichen peu fréquent

69. Lobe mesurant gênéralement plus de $2 \mathrm{~mm}$ de large; revers noir au centre (brun à la périphérie) . . . . . . . . . . . . . . . . . . 70 .

70. Larges lobes ronds, de 6 à $15 \mathrm{~mm}$ de large, souvent pourvus de cils noirs et filiformes s'insérant à la marge; revers partiellement dénudé (c.-à-d., dépourvu de 
rhizines) en périphérie; médulle koH+ jaune (sans tourner au rouge), $\mathrm{Pd}+$ jaune. Espèce rare; croît sur l'écorce... . . . . . . . . . . Parmelia crinita

70. Lobes fins et "équarries", dont la surface est couverte d'un réseau de crêtes; présence de rhizines jusqu'au pourtour du thalle; médulle KoH+ jaune, virant au rouge sang, Pdt jaune. . . . . . . . . . . . . . . 71 .

71. Rhizines squarreuses (fig. 65). Espèce très commune; pousse surtout sur l'écorce . . . . . . . Parmelia squarrosa

71. Rhizines simples (non ramifiées) ou dichotomes. Taxon rare; habituellement saxicole.......... . Parmelia saxatilis

72. Sorédies disposées surtout à la marge ou à l'extrémité des lobes. . . . . . . . . . . . . . . . 73.

72. Sorédies distribuées surtout en plaques ou sur les crêtes à la surface du thalle, de même que parfois en périphêrie . . . . . . . . . . . . . . . . . 89.

73. Médulle jaune moutarde ou orangé vif ............ . 74 .

73. Médulle blanche ou jaune très pâle . . . . . . . . . . . . 75 .

74. Thalle comme givré car recouvert d'une pruine blanche et poussiéreuse, parfois uniquement à l'extrémité des lobes; médulle jaune moutarde; lobes de 1 à $2 \mathrm{~mm}$ de large; toutes les sorédies en position marginale. Lichen peu fréquent; pousse sur l'écorce dans les forêts décidues parvenues à maturité . . . . . . Pyxine sorediata

74. Pas de pruine sur le thalle; médulle orange vif (quelquefois blanche chez certains lobes); lobes de moins de $1 \mathrm{~mm}$ de large; sorédies marginales et superficielles. Taxon commun sur l'écorce de toutes sortes d'arbre...... (fig. 44) Phaeophyscia rubropulchra

75. Thalle pruineux, quoique de temps en temps seulement à l'extrémité des lobes; sorédies entièrement marginales; revers noir; rhizines squarreuses; médulle et cortex insensibles à $\mathrm{KOH}$. . . . . . . . . . . . . . . . 76 . 75. Thalle complètement dépourvu de pruine; médulle KoH+ ou KOH- . . . 77. 
76. Médulle blanche, KOH- . Plante commune; fréquemment rencontrée sur les ormes et les peupliers au bord des routes, mais rarement sur les rochers . . . . . Physconia detersa 76. Médulle et sorédies jaunâtre pâle, KoH+ jaune (parfois dêlavé). Taxon rare; crô̂t sur les arbres le long des chemins . . . . . . . Physconia cf. enteroxantha (dont la différenciation de $P h$. detersa est douteuse).

77. Lobes larges, arrondis, dont la largeur excède $3 \mathrm{~mm}$; médulle C+ rouge ou rose; thalle maculé de blanc (pseudocyphelles) . . . . 78.

77. Lobes non arrondis, dont la largeur n'atteint pas $3 \mathrm{~mm}$;

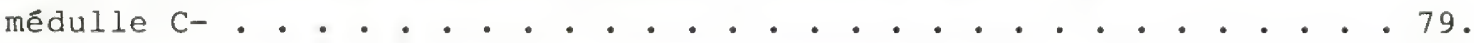

78. Sorédies entièrement situées en position marginale; pourtour du revers présentant une large plage dénudée. Espèce fréquente; colonise les rochers moussus partiellement à l'ombre, surtout les falaises . . . . . . . . . . . . . . . . . Cetrelia olivetorum

78. Sorédies disposées à la surface du thalle (superficielles) tout autant que sur les rebords; rhizines couvrant la face inférieure presque jusqu'au contour des lobes. Espèce rare; vit sur l'écorce . . . . . . . . . . . . . . . . . . Parmelia borreri

79. Thalle gris clair à vert grisâtre; cortex supérieur koH+

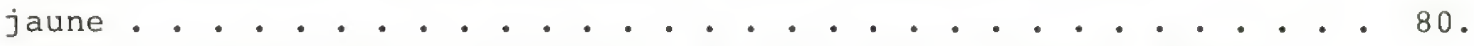

79. Thalle brun olivâtre ou grisâtre, ou bien vert grisâtre foncé (gris clair seulement chez Physcia chloantha); cortex supérieur KOH- • • • • • • • • • • • • • • • • • • . . . 83 .

80. Extrémité des lobes formant des structures nettes cucullées ou capitées, pleines de sorédies granuleuses; cils habituellement visibles à l'extrémité des lobes. Lichen commun; s'installe surtout sur les arbres au bord du chemin aussi bien que sur les rochers calcaires • . . . . . . . . . . . . . . . . . Physcia adscendens

80. Extrémité des lobes plus ou moins aplanie, en forme ni de capuchon, ni de casque . . . . . . . . . . . . . 81 . 
81. Grosses sorédies devenant des granules, ne se retrouvant, dans la plupart des cas, que sur le rebord des lobes; lobes finement découpés. Plante très commune; pousse sur l'écorce d'arbre, surtout l'orme, le peuplier et le frêne et, parfois, sur les rochers........... . (fig. 45) physcia millegrana

81. Sorédies formant des plaques farineuses se retrouvant presque entièrement sur le revers de l'extrémité légèrement élargie des lobes (labriforme); lobes non finement découpés . . . . . . 82 . (N.B.: Parmeliopsis hyperopta, souvent sorédié sur la face supérieure de l'extrêmité des lobes, peut être identifié ici. Se référer au doublet 97.)

82. Rhizines ramifiées, blanches ou brun jaunâtre clair, ou encore cils ressemblant à des rhizines se prolongeant souvent au-delà du contour des lobes. Plante peu fréquente; pousse sur l'écorce. . (fig. 46) Heterodermia speciosa

82. Pas de cils marginaux, ramifiés, semblables à des rhizines. Taxon peu fréquent; ordinairement saxicole, quoiqu'il croisse de temps en temps sur les arbres près du bas-côté des routes... . . . . . . . . Physcia dubia

83. Revers blanc; soralies surtout labriformes et farineuses, quelques-une étant marginales; lobes atteignant 0,4 à $0,7 \mathrm{~mm}$ de large. Lichen peu fréquent; se retrouve beaucoup sur l'écorce et exceptionnellement sur le roc . . . . . Physcia chloantha

83. Revers noir; soralies marginales ou terminales, non labriformes . . . . . . . . . . . . . . . 84 .

84. Lobes plutôt larges, aux extrémités concaves, ordinairement de 0,8 à $3 \mathrm{~mm}$ de large; soralies rondes, capitées, marginales à submarginales et superficielles; longues rhizines se prolongeant au-delà du rebord des lobes. Plante rare; se rencontre sur l'écorce

84. Lobes étroits, de moins de $1.5 \mathrm{~mm}$ de large; soralies $\mathrm{ni}$ submarginales, ni superficielles; rhizines longues ou courtes . . . . . . . . . . . . . . . . 85 .

85. Jeunes lobes et bord des apothêcies souvent pourvus de minuscules poils incolores, c.-à-d., translucides; sorédies 

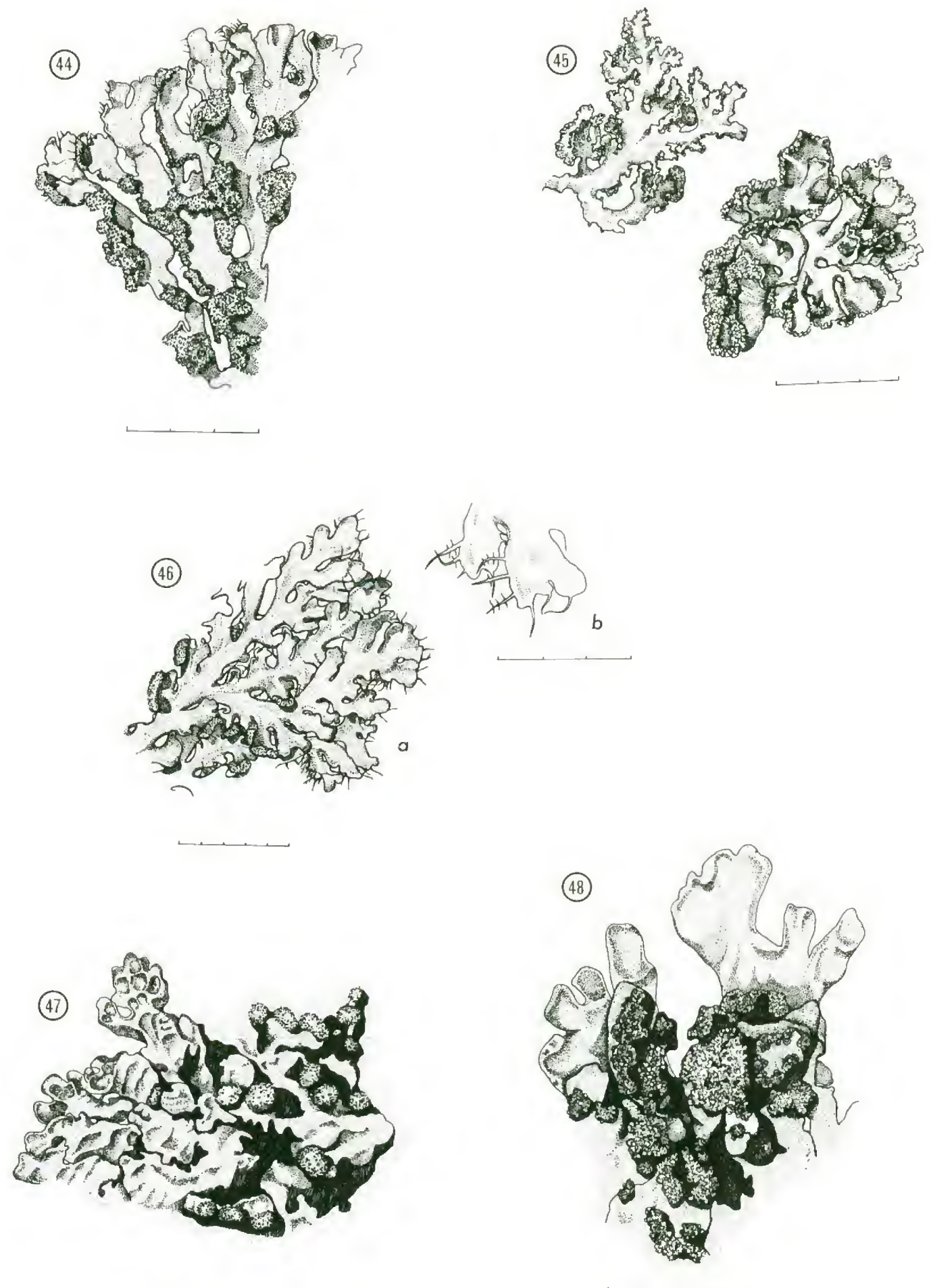

Figures 44 à 48.-44. Phaeophyscia rubropulchra. -45. Physcia millegrana: a) et b) sont deux morphotypes. -46 . Heterodermia speciosa: a) thalle; b) cils marginaux. -47.Parmelia sorediosa. 48. Parmelia disjuncta. Échelle: chaque division $=1 \mathrm{~mm}$. 
verruciformes, uniquement marginales. Taxon peu fréquent; crôt habituellement sur le roc, exceptionnellement sur l'écorce . . . . . . . . . . . . Phaeophyscia cernohorskyi 85. Lobes et marges d'apothếcies sans poils incolores . . . . . . . . 86.

86. Espèce saxicole (croissant de temps en temps sur les rochers moussus) . . . . . . . . . . . . . 87.

86. Espèce se développant sur l'écorce ou le bois soralies réparties en plaques farineuses, presque sphériques, d'olive foncé à noir jaunâtre, plus ou moins portées sur le bout de lobes courts; pourtour des lobes et apothécies souvent cilié ou frangé de rhizines. Taxon fréquent... . . . . . . . . . Phaeophyscia pusilloides

87. Coloration du thalle brun foncé à brun olivâtre; soralies rondes à subsphériques, fréquemment surélevées sur de courts lobes latéraux ou terminaux; sorédies verdâtres, farineuses. Plante peu fréquente, colonise le granite ou d'autres roches dures dans les stations ensoleillées . . (fig. 47) Parmelia sorediosa

87. Thalle brun grisâtre à olive; sorédies jamais surélevés ou en plaques subsphériques; sorédies verruciformes à isidifères . . . 88 .

88. Lobes thallins aplatis ou bombés, atteignant une largeur variant entre 0,15 et $0,5 \mathrm{~mm}$; abondantes sorédies isidifères, sombres, ordinairement en touffes terminales ou marginales. Taxon rare; normalement uniquement saxicole... . . . . . . Phaeophyscia sciastra

88. Lobes thallins de 0,6 à $1,5 \mathrm{~mm}$ de large, concaves aux extrémités (comme chez Phaeophyscia hispidula), plutôt luisants et lisses. Plante fréquente . . . . . . . . . . . . . . Phaeophyscia adiastola

89. Thalle subcrustacé, très étroitement soudé au substrat; rhizines presque absentes; menus lobes gris brunâtre, moins de 0,4 mm de large; cortex кон-. Lichen rare; se développe sur l'écorce. . . . . . . . . . . Physciopsis adglutinata

89. Thalle foliacé, adhérant lâchement au support (dont il est facilement détachable); largeur des lobes dépassant 0,5 mm . . . . 90 . 90. Thalle sec brun à brun olivâtre..... . . . . . . . . 91 . 
90. Thalle gris clair ou parfois brunâtre à la périphérie . . . . 93.

91. Lobes thallins plats, minces; sorédies en plaques très irrêgulières, provenant de granules isidifères; médulle C+ rouge. Plante commune; pousse sur l'écorce... Parmelia subaurifera

91. Lobes étroits, de temps en temps convexes, ordinairement épais; sorédies se limitant à des amas ronds individualisés; médulle C- ou C+ jaunâtre. Espèce saxicole . . . . . . . . 9 92.

92. Sorédies farineuses, réellement disposées à l'extrémité de courts lobes ascendants et par conséquent, paraissant légèrement surélevées. Taxon peu fréquent . . . . . . . . . . . . (fig. 47) parmelia sorediosa

92. Sorédies non surélevées, nettement superficielles, venant de grosses granules noires, semblables à des isidies. Plante rare ...... (fig. 48) parmelia disjuncta

93. Gros lobes dont la largeur excède $4 \mathrm{~mm}$; médulle c+ rouge; sorédies également marginales (voir doublet 78) . . Parmelia borreri 93. Largeur des lobes n'atteignant pas $4 \mathrm{~mm}$; médulle C- . . . . . . . 94.

94. Sorédies s'étalant le long de veines disposés en filet à la surface; médulle blanche, $\mathrm{KOH}+\mathrm{j}$ aune puis rouge. Lichen très commun sur toutes sortes d'arbres, le bois et parfois les rochers ........ (fig. 49) parmelia sulcata 94. Sorédies disposées en plaques rondes ou irrégulières . . . . 95.

95. Lichen croissant sur les écorces d'arbres ou le bois . . . . . . 96. 95. Espèce saxicole . . . . . . . . . . . . . . . . 98.

96. Largeur des lobes n'atteignant pas $2 \mathrm{~mm}$; médulle blanche; petites plaques rondes de sorédies à la surface; thalle très intimement lié au substrat . . . . . . . 97.

96. Largeur des lobes excédant $2 \mathrm{~mm}$; thalle gris, non tacheté de blanc; sorédies réparties en petites surfaces irrégulières; médulle jaune pâle, particulièrement près de la couche algale. Espèce commune, pousse sur l'écorce.............. . . . . . Parmelia aurulenta 
97. Revers luisant, brun pâle à foncé; fines sorédies

(farineuses) blanches à noir verdâtre, provenant de plaques

érodēes et souvent situées près de l'extrémité des lobes;

spores incolores et non cloisonnées. Espèce rare; pousse sur

les rameaux de résineux et le bois mort. . . Parmeliopsis hyperopta

97. Revers mat allant de blanc à une teinte claire de chamois

crème; sorédies verruciformes, dont la coloration varie entre

blanc et un gris à reflet bleuté, surgissant du cortex

supérieur, empruntant la forme de plaques rondes, devenant

parfois confluentes, jamais disposées près de l'extrémité des

lobes; spores brunes, uniseptées. Espèce rare; s'installe

sur l'écorce d'espèces décidues . . . . . . . . Physcia tribacoides

98. Médulle KOH+ jaune; thalle blanc bleuâtre ou gris, tachê

de blanc, pourvu de plaques blanches de sorédies

individualisées et globuleuses. Taxon peu fréquent;

colonise habituellement le calcaire ou associé aux

perchoirs d'oiseaux . . . . . . . . . . . . Physcia caesia

98. Médulle KOH-; thalle gris clair; largeur des lobes

n'atteignant pas $0,5 \mathrm{~mm}$. Lichen rare; crô̂t

généralement sur le roc ne réagissant pas à une

application de $\mathrm{HCl}$. . . . . . . morphotype de Physcia dubia

99. Lichen terricole . . . . . . . . . . . . . . . . . 100.

99. Lichen saxicole, corticole ou lignicole . . . . . . . . . . . . 102 .

100. Largeur des lobes excédant $20 \mathrm{~mm}$; face supérieure pourvue ici et là de petites céphalodies brunes et

plates; revers feutré et décortiqué. En règle générale, espèce de milieux humides et moussus . . . . . . . . . . . 101.

100. Lobes ténus (moins de $5 \mathrm{~mm}$ de large), ascendants, dichotomiques, formant des coussins subfruticuleux; couleur thalline allant de brun à brun olivâtre. Lichen rare, s'installant sur de minces sols secs et rocailleux ............... (... (fig. 50) Cetraria arenaria

101. Face inférieure colorẻe d'une teinte plus ou moins uniforme de noir brunâtre et pourvue ou dépourvue de veines plus ou moins accentuées; face inférieure des apothécies totalement cortiquée. Espèce fréquente... . . . . . . . Peltigera aphthosa 

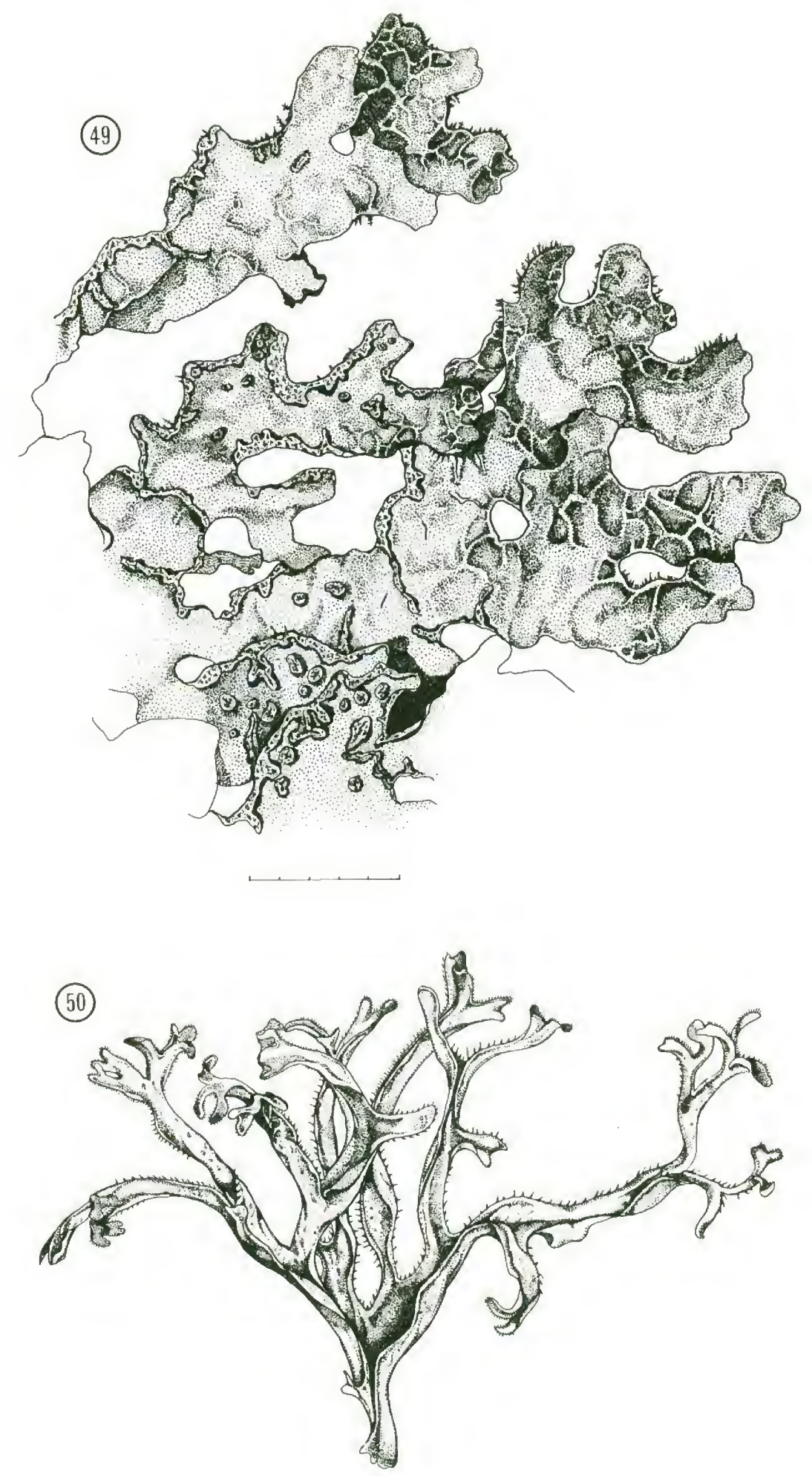

Figures 49 et 50.-49. Parmelia sulcata. -50. Cetraria arenaria. Échelle: chaque division $=1 \mathrm{~mm}$. 
101. Veines saillantes, brunes à noires, courant sur la face inférieure; face inférieure des apothécies parsemée

d'arēoles cortiquées. Espèce rare. . . . . Peltigera leucophlebia

102. Thalle brun, brun olivâtre ou gris verdâtre sombre, mais rarement gris clair; face supérieure KOH- . . . . . . . . . . 103.

102. Thalle blanc grisâtre, gris clair ou gris nuancé de bleu; à la face supérieure, KoH+ jaune . . . . . . . . . . 113.

103. Nombreux petits lobules plans naissant à la périphérie des lobes (lobules un peu cylindriques parfois, ce qui les fait ressembler à des isidies) . . . . . . . . . . . . . . . . . 104 .

103. Lobes thallins dépourvus de lobules de quelque sorte que ce soit........................... . . 106.

104. Thalle tout entier fortement pruineux, surtout sur les lobules; revers entièrement blanc ou chamois très pâle, ne noircissant pas; apothêcies abondantes, à marges pourvues de prolongements sous forme de lobules radiés. Plante peu commune; se développe sur l'écorce et exceptionnellement sur les rochers moussus . . . . . . . . . . . . . . . . . Physconia pulverulacea

104. Thalle sans pruine, ou un peu scabré, ou encore couvert de taches pruineuses éparpillées à l'extrêmité des lobes; revers blanc en périphérie, mais devenant toujours noir vers le centre du thalle; si la marge des apothécies est lobulée, alors elle n'est pas rađiée. . . . . 105.

105. Lobes thallins longs, plus ou moins linêaires, pourvus de lobules élancés, parfois presque cylindriques (v. fig. 65), qui sont marginaux et superficiels; thalle ordinairement brunâtre ou olive sombre; rhizines squarreuses sur les spécimens bien développés. Lichen peu fréquent; rencontré sur les arbres ou quelquefois sur les rochers moussus

105. Lobes ronds, irréguliers, un peu imbriqués; thalle gênéralement gris ou gris veraâtre, parfois légèrement brunâtre; jamais de rhizines squarreuses. Espèce peu commune; se trouve sur les rochers moussus et très rarement au pied des arbres . . . . . . . . . . . Phaeophyscia imbricata 

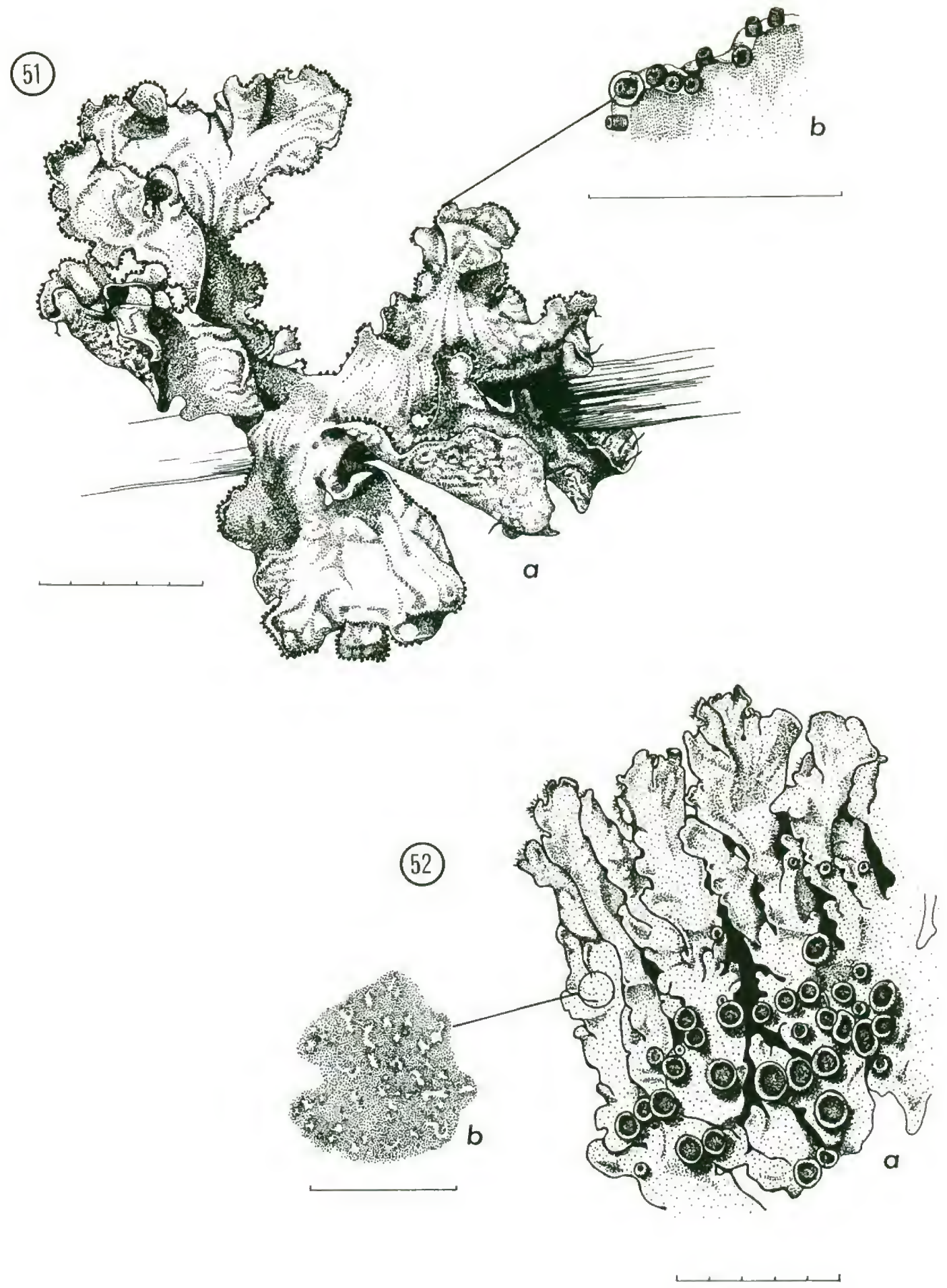

Figures 51 et 52.-5l. Cetraria halei: a) thalle; b) pycnides.-52. Physcia aipolia: a) thalle; b) taches blanches (macules) à la surface. Échelle: chaque division $=1 \mathrm{~mm}$. 
106. Présence d'apothécies ou pycnides le long de la marge des lobes ou près de celle-ci (v. fig. 5l); rhizines clairsemées. Taxon croissant ordinairement sur l'écorce et les branches de conifères, d'aulne ou de bouleau . . . . . 107.

106. Apothécies ou pycnides présentes ou absentes, non marginales; abondance de rhizines. Plante s'installant sur différents substrats . . . . . . . . . . . 109.

107. Thalle de grande taille, 2 à $4 \mathrm{~cm}$ de large; apothécies se développant sur le revers de l'extrémité des lobes, qui se retrousse, créant ainsi l'impression que les fructifications sont sur la face supérieure . . . . . . . . . . . . 108.

107. Thalle de très petite taille n'atteignant pas $2 \mathrm{~cm}$ de large; apothécies prenant naissance à la face supérieure des lobes. (Rechercher les jeunes apothécies.) Espèce rare. . Cetraria sepincola

108. Médulle C-, KC+ rose, et dégageant une fluorescence brillante et blanche, si on l'expose à une lumière ultra-violette. Lichen commun ..... (fig. 51) Cetraria halei 108. Medulle $\mathrm{C}+$ rose, $\mathrm{KC}+$ rouge mais UV-. Plante rare Cetraria ciliaris

109. Lobes thallins de 2 à $5 \mathrm{~mm}$ de large, fixés lâchement; revers brun, luisant; à l'état sec, coloration du lichen ordinairement olive brunâtre, plus ou moins luisant; médulle Pdt rouge. Taxon corticole. . . . . . . . . . . . 110 .

109. Lobes thallins de 0,5 à $2 \mathrm{~mm}$ de large; revers noir et mat; thalle mat, brun foncé à brun grisâtre, plat et fermement fixé au substrat; médulle Pd- . . . . . . . . . . . . . Ill.

110. Thalle lisse; pseudocyphelles absentes ou très clairsemées; apothécies plates à maturité, dotées d'une mince marge lisse et évanescente. Espèce peu commune . . . . . . . . . . . . . Parmelia septentrionalis

110. Thalle rugueux, plissé; pseudocyphelles généralement visibles, particulièrement sur le contour des apothécies; apothécies cupuliformes, à marge persistante et souvent finement crénelée. Espèce rare . . Parmelia olivacea 
111. Lichen saxicole; lobes élancés de moins de $0,6 \mathrm{~mm}$ de large, normalement brun foncé; thalle ni verruqueux, ni pulvérulent, ordinairement stérile. Taxon rare...... . Phaeophyscia decolor

1ll. Lichen corticole ou lignicole (exceptionnellement saxicole); lobes plus larges, de 0,4 à $1,5 \mathrm{~mm}$ de large; thalle vert-de-gris foncé à gris brunâtre, souvent verruqueux, ordinairement fertile, muni de rhizines naissant à la base du

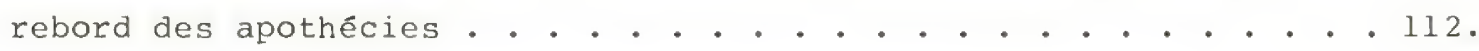

112. Fins poils incolores et raides, garnissant au moins l'extrémitê de quelques lobes et la marge des apothécies. Espèce fréquente. . . . . Phaeophyscia hirtella 112. Espèce dépourvue de poils ....... Phaeophyscia ciliata

113. Face inférieure brun jaunâtre clair, "duveteuse" (tomenteuse), pourvue ou non de rhizines clairsemés; médulle Ct rose, surtout au-dessous de la couche algale; thalle très grand, au diamètre variant souvent entre 12 et $15 \mathrm{~cm}$; largeur des. lobes excédant souvent $10 \mathrm{~mm}$; grosses apothécies brunes et, en règle générale, abondantes; pas de pseudocyphelles et de lobules. Lichen rare; se rencontre dans les peuplements riches ou près des tourbières, sur l'écorce... . Lobaria quercizans

113. Face inférieure soit garnie de rhizines, soit entièrement dénudée, non tomenteuse; médulle C- . . . . . . . . . . 114.

114. Thalle volumineux, strié, plissê et profondément grêlé; face inférieure luisante, presque complètement dépourvue de rhizines, maculée de blanc, de brun et de noir. Taxon peu commun, poussant sur le bois ou l'écorce de résineux . . . . . . . . . . . platismatia tuckermanii

114. Thalle plus ou moins aplani, ni strié, ni plissé; coloration de la face inférieure uniforme (c.-à-d., pas de maculage) . . . . . . . . . . . . . 115.

115. Revers noir au centre, brun foncé au bord; médulle ordinairement jaune pâle, au moins près de la couche algale, $\mathrm{kOH}+$ jaune foncé à orange. Plante commune; pousse sur l'écorce... . . . . . . . . . . . Parmelia galbina 115. Revers tout entier brun jaunâtre clair à blanc . . . . . . . 116. 
116. Pseudocyphelles normalement visibles à la surface des jeunes lobes; des points noirs (les sommets des pycnides qui sont enfoncés dans le thalle) se trouvent fréquemment sur la face supérieure du thalle; revers brun, luisant, totalement cortiqué; lobes principaux larges, de 2 à $5 \mathrm{~mm}$ de large, et abondance de lobules ténus sur tout le thalle; abondance de grosses apothécies marron, luisantes et profondément concaves; médulle $\mathrm{KOH}-$. Espèce rare; crốt sur l'écorce

116. Pas de pseudocyphelles ni de pycnides; revers au moins blanc en partie et dépourvu de cortex; lobes surtout étroits, de l à $3 \mathrm{~mm}$ de large, s'évasant un peu au bout. . . 117.

117. Lobules s'insérant abondamment à la marge des lobes et des apothécies; apothécies fréquentes, très déprimées, non pruineuses; médulle кон+ jaune pâle. Espèce rare

117. Pas de lobules; apothécies de 1 à $2 \mathrm{~mm}$ de diamètre, aplanies à légèrement concaves, brun foncé à presque noir, souvent couvertes de pruine... . . . . . . . . . . . 118 .

118. Médulle KoH-; face supérieure dotée d'un gris plus ou moins uniforme, non tachetée; taxon fréquent; pousse sur les arbres en bordure des routes, surtout l'orme

118. Médulle KoHt jaune; face supérieure visiblement tachée de blanc.................... . . . . . . . 9.

119. Lichen très commun sur l'écorce, surtout celle des ormes et des peupliers. Thalle blanc à blanc tirant sur le gris ................ (. . (fig. 52) physcia aipolia

119. Lichen peu fréquent, saxicole. Thalle gris bleuâtre à gris violacé . . . . . . . . . . . . . . . . Physcia phaea 


\section{LES LICHENS FRUTICULEUX}

\section{Synopsis}

1. Tige ou branches nettement creuses . . . . . . . . . . . . . . . . 2 .

1. Tiges ou branches pleines, mais mëdulle parfois lâche et cotonneuse . . . . . . . . . . . . . . . . . . . . . . . . . . 49.

2. Podétions fortement ramifiées, formant des coussins, ou touffes, buissonneux . . . . . . . . . . . . 3. (p. 109)

2. Podétions pas du tout rameux ou, tout au plus, ramifiés une ou deux fois, à l'allure jamais "buissonneuse" . . . . . . . 8.

8. Podétions se terminant par des fructifications (apothécies) vermeilles. . . . . . . . . . . . . . . . . . . 9. (p. 110)

8. Podétions soit pourvus d'apothécies brunes, soit dépourvus

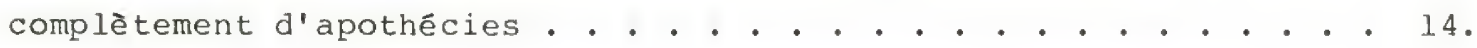

14. Podétions munis de coupes plus ou moins individualisées ou de scyphes superposés les uns aux autres, plats et discoïdes . . . . . . . . . . . . . . 15. (p. 112)

14. Podétions sans coupe, soit atténués en pointes, soit pourvus de fructifications au sommet . . . . . . . . . . . 33 .

33. Podétions dépourvus de sorédies ou de granules . . . • • 34. (p. 115)

33. Podétions plus ou moins sorédiés ou granuleux . • • • • • . 40. (p. 117)

49. Branches ou tiges à croissance verticale dirigée vers le haut, naissant souvent d'une croûte granuleuse ou poussiēreuse . . . . . . . . . . . . . . . 50. (p. 119)

49. Branches ou tiges poussant perpendiculairement à une surface verticale, ou pendantes, ne provenant jamais d'une croûte basilaire . . . . . . . . . . . . . . . . 53.

53. Une coupe transversale rẻvèle la rondeur presque parfaite des rameaux; branches plus ou moins filamenteuses . . . . . . . 54. (p. 120)

53. Une coupe transversale présente des rameaux nettement aplanis ou très irréguliers, au moins à la base . • . • • • • • • . 58. (p. 122) 


\section{Clef Générale}

1. Tiges ou branches nettement creuses . . . . . . . • . . . . . . . 2 .

1. Tiges ou branches pleines, mais médulle parfois lâche et cotonneuse . . . . . . . . . . . . . . . . . . . . . . . . . 49.

2. Tiges (podétions) fortement ramifiées, formant des coussins, ou touffes, buissonneux (fig. 53, 54) . . . . . . . . 3.

2. Podétions pas du tout rameux ou, tout au plus, une ou deux fois ramifiés, à l'allure jamais "buissonneuse" (fig. 55-58) . . . . . . . . . . . . . . . 8.

3. Podétions garnis de squamules clairsemées à denses, particulièrement à la base; pàt rouge . . . . . . . . . . . . . . . . 4 .

3. Podétions totalement dépourvus de squamules . . • • • . . . . . . . 5.

4. Podétions très rameux ordinairement, tout à fait dépourvus de sorédies. Espèce peu fréquente, crô̂t généralement sur le sol à l'ombre (coloration vert-de-gris foncé), et parfois à la lumière (couleur vert brunâtre) . . . . . . . . . . . . Cladonia furcata

4. Podétions peu branchus, ramifiés surtout dans la partie superieure; extrémité des rameaux pourvue de sorédies grossières ou granuleuses. Espèce peu fréquente, se développe à terre et sur les tronçons d'arbre . . . . . . . . . . . . . . Cladonia scabriuscula

5. Thalle gris argenté ou gris bleuâtre; extrémités des rameaux, règle gênérale, brunies et recourbées plus ou moins du même côté; surface KOH+ jaune, Pd+ rouge. Lichen commun; hëliophile, se trouve sur les sols minces, les rochers et parmi les mousses . Cladina rangiferina (véritable lichen des caribous)

5. Thalle nettement vert jaunâtre ou gris verdâtre (jamais gris argentê); surface $\mathrm{KOH}_{-}^{-}$et $\mathrm{Pd}-$. . . . . . . . . . . . . . . . . . 6.

6. Thalle se développant en touffes rondes, fortement rameuses, de 2,5 à $5,0 \mathrm{~cm}$ de large; extrémité de chaque branche terminée par un verticille étoilé fait de 4 ou 5 rameaux miniscules entourant une ouverture centrale. Plante peu fréquente; terricole vit en pleine lumière .............. (fig. 53) cladina stellaris 
6. Plante ne se développant pas en touffes rondes, ou à extrêmité des rameaux non verticillée . . . . . . . . . 7 .

7. Podétions lisses, luisants, très jaunâtre; extrémitếs divergentes, dressées et très obtuses. Taxon commun; crô̂t sur le sol au soleil ou sur le roc . . . . . . . Cladonia uncialis

7. Podétions uniformément mats en surface, ordinairement verdâtre ou lëgèrement jaunâtre; bouts divergents mais souvent recourbés. Taxon commun; normalement héliophile, s'installe sur le sol ou sur un tapis de mousses

(fig. 54) Cladina mitis

8. Podétions se terminant en apothécies vermeilles . . . . . . . . 9.

8. Podétions soit pourvus d'apothécies brunes, soit dépourvus complètement d'apothécies . . . . . . . . . . . . 14 .

9. Podétions couverts de sorédies . . . . . . . . . . . . . . . 10.

9. Podétions non sorédiês, caractères très variables: podétions non ou à peine rameux, envahis de squamules ou non, jaunâtre ou parfaitement gris. Espèce très fréquemment rencontrēe sur le sol, les tronçons d'arbre, au pied des arbres . . . . . . (les petits soldats) (fig. 6) Cladonia cristatella

10. Podétions se terminant en coupes distinctes (scyphes), munis d'apothécies rouges s'insérant à la marge les coupes; $\mathrm{KOH}-$. . . . . . . . . . . . . . . . . . . . . . . . 11 .

10. Podétions dépourvus de coupes, se terminant en pointes émoussées ou aiguës . . . . . . . . . . . . . . . . 13.

11. Podétions KOH+ jaune vif, Pdt orange. Lichen rare; terricole ou lignicole (v. doublet 15) . . . . . . . . Cladonia digitata

11. Podétions KOH- et $\mathrm{Pd}-$. . . . . . . . . . . . . . . . . . . 12 .

12. Scyphes souvent élancés, un peu échancrés longitudinalement; sorédies d'aspect poussiéreux, très fines. Espèce commune, colonisant le sol et les tronçons d'arbre . . . . . . . . . . . Cladonia deformis

12. Scyphes en forme de coupe, non échancrés; sorédies grossières granuleuses, farineuses. Espèce fréquente; terricole. . . . . . . . . . . . . . Cladonia pleurota 

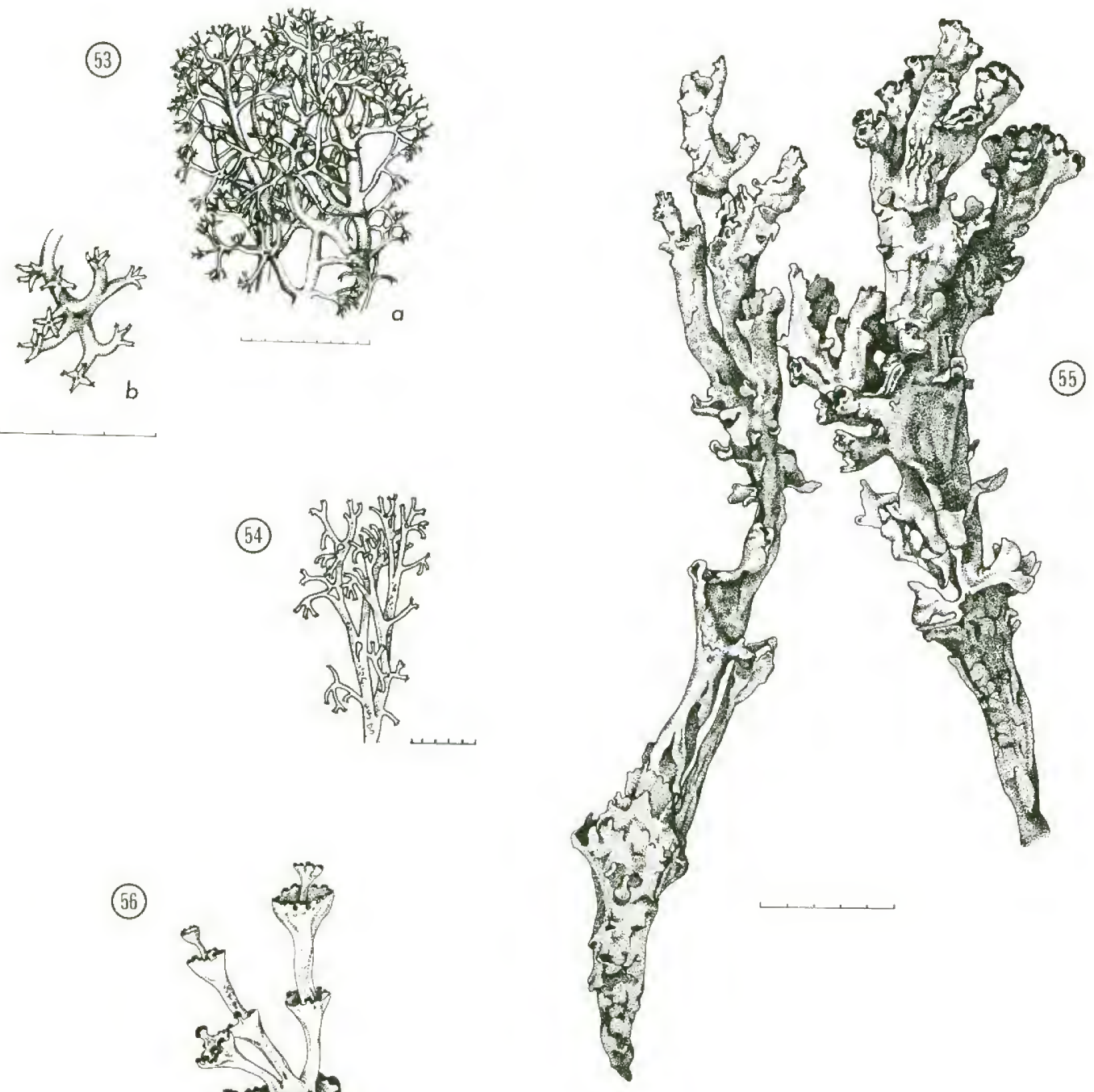

(56)
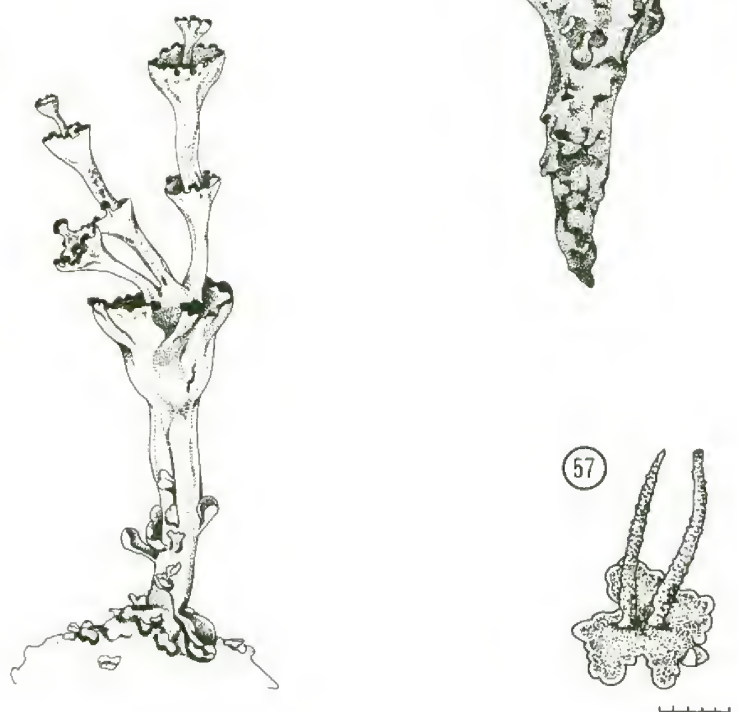

(58)

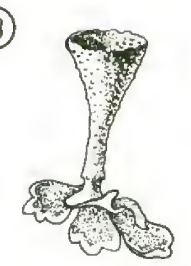

Figures 53 à 58.-53. Cladina stelzaris: a) thalle; b) grossissement de l'extrémité d'une branche.-54. Cládina mitis.-55. Cladonia

phyllophora.-56. Cladonia cervicornis ssp. verticillata.-57.

Cladonia coniocraea.-58. Cladonia chlorophaea 8. l. Échelle: chaque division $=1 \mathrm{~mm}$. 
13. Podētions KOH+ jaune foncé, Pd+ orangé; structures ordinairement courtes, épaisses et robustes. Plante rare; vit à même la terre, les tronçons d'arbre et le roc

13. Podétions KOH-, Pd-, généralement effilês. Taxon commun, se développe sur le sol, les tronçons d'arbre et au pied des arbres . . . . . . . . . . . . . cladonia bacillaris

14. Podétions munis de coupes plus ou moins individualisees ou de scyphes superposés les uns aux autres, plats et discoïdes . . . . . . . . . . . . . . . . . 15.

14. Podétions sans coupes ou sans étages, soit atténués en pointes, soit pourvus de fructifications au sommet . . . . 33 .

15. Podêtions $\mathrm{KOH}+$ jaune vif, envahis de sorédies d'aspect

farineux; rebord des scyphes souvent involuté et parfois

êchancré. Espèce rare, recherchant les troncs d'arbres et le sol . . . . . . . . . . . . . . . cladonia digitata

15. Podétions KOH- ou, $\mathrm{KOH}+$ plus ou moins brun terne; structures sorédices ou non . . . . . . . . . . . . . . . 16 .

16. Podétions nettement jaunâtre (habituellement vert jaunâtre pâle), sorédiés; Pd- . . . . . . . . . . . . 17 .

16. Podétions dépourvus de nuance de jaune; Pd- ou Pd+ rouge

17. Sorédies grossières, granuleuses ou farineuses; scyphes évasés, non échancrés. Taxon fréquent; terricole. . Cladonia pleurota

17. Sorédies d'aspect farineux; scyphes souvent allongês, un peu échancrés dans le sens de la longueur. Taxon peu fréquent, croît sur les tronçons d'arbre et la terre... . . Cladonia deformis

18. Scyphes perforés d'ouvertures béantes donnant sur l'intérieur des pođétions . . . . . . . . . . . . . . . 19. 18. Scyphes complètement fermés, non perforés . . . . . . . 24.

19. Sorédies d'aspect farineux répandues sur les podétions dépourvus de squamules; coloration très pâle, presque blanche; marge des coupes à peine involutée; KOH- et Pd-. Lichen fréquent sur la terre et les tronçons d'arbre . . Cladonia cenotea 
19. Podétions pas du tout sorédiés . . . . . . . . . . . 20.

20. Thalle Pd-, devenant très fluorescent s'il est exposé à la lumiēre ultra-violette de grande longueur d'onde . . . . . 21.

20. Thalle Pd+ rouge, réaction négative à l'ultraviolet; podétions non squamuleux ou pourvus de squamules souvent lobées mais non finement dẹcoupées . . . . . . . . 22 .

21. Surface des pođétions (cortex) discontinue, inégale formant d'abondantes petites squamules finement découpés, de moins de $2 \mathrm{~mm}$ de long, étalées sur presque tout le podétion; scyphes simples, souvent trapus (quelquefois larges et prolifères). Taxon commun, rencontré ordinairement dans les stations partiellement ombragées, sur les tronçons d'arbres et le sol moussu . . . . . . . . . . . . . Cladonia squamosa

21. Cortex continu, lisse, rarement garni de squamules; scyphes à prolifération ordinairement marginale, ce qui donne naissance à des coupes superposées les unes aux autres. Lichen terricole peu fréquent, habituellement de milieux ouverts................. cladonia crispata

22. Squamules basilaires très grandes, ascendantes, souvent de plus de $6 \mathrm{~mm}$ de long; podétions vert sombre, pourvus de scyphes très irréguliers qui sont souvent fendus longitudinalement. Plante peu fréquente; terricole

Cladonia turgida

22. Squamules basilaires de petite taille, dépassant rarement $3 \mathrm{~mm}$ de long................ 23 .

23. Pođêtions gênéralement dotés de scyphes aisément reconnaissables, perforés; cortex des plus vieilles sections de podétions lisse, uniformément foncé. Taxon fréquent; terricole................ . . . . Cladonia multiformis

23. Podétions en forme de coupes irrégulières, souvent squamuleuses en périphérie, percées de perforations et de lacérations de forme irrégulière; cortex des vieilles parties se défaisant par petites plaques blanches dévoilant ainsi un fond noir. Espèce commune; terricole.. (fig. 55) Cladonia phyllophora 
24. Podétions sorédiés . . . . . . . . . . . . 25.

24. Podétions non sorêdiés; Pdt rouge . . . . . . . . . . 29 .

25. Scyphes grêles ou s'évasant brusquement au sommet d'un

podétion élancé, peu profonds, disparaissant parfois

totalement et ne laissant qu'un podétion pointu . . . . . . . 26 .

25. Scyphes étroits ou larges, plus ou moins profonds, résultant

gênéralement d'un élargissement graduel du podétion;

podétions jamais pointus . . . . . . . . . . . . 27 .

26. Sorédies abondantes, très fines; podétions trapus,

rarement effilés, prenant naissance au centre de grosses

squamules basilaires, ordinairement non lobés; pas de

prolifération marginale; Pdt rouge. Lichen très commun;

croît sur le bois, au pied des arbres et de temps à

autre sur le sol... . . . . (fig. 57) Cladonia coniocraea

26. Sorédies d'aspect granuleux, éparses; podétions grêles,

pourvus de squamules basilaires lobées ou finement

découpées; scyphes à prolifération souvent marginale et

à l'allure étoilée; Pd- ou Pd+ rouge. Lichen commun; se

dêveloppe surtout sur la terre au soleil. . . . . Cladonia rei

27. Sorêdies d'aspect farineux; Pdt rouge . . . . . . . . . 28 .

27. Podétions couverts de sorédies grossières ou granuleuses;

scyphes larges, profonds; Pd- ou Pd+ rouge. Espèce très

commune; se rencontre sur le sol, les tronçons d'arbre et au

pied des arbres......... (fig. 58) Cladonia chlorophaea

(N.B.: Quatre "micro-espèces", distinguées les unes des

autres surtout grâce au chimisme du thalle, s'identifient

ici: Cladonia merochlorophaea contenant de l'acide

mérochlorophaeique, $C$. grayi renfermant de l'acide

grayanique, C. cryptochzorophaea élaborant I'acide

cryptochlorophaeique et $C$. chlorophaea s.s. qui est

dépourvu de tous ces acides-là. Exception faite de quelques

spécimens de $C$. grayi, toutes ces espẽces produisent de

l'acide fumarprotocétrarique et se teintent de rouge à la

suite d'une application de Pd. C. chtorophaea s.s. est le

plus commun.)

28. Podétions totalement sorédiés; scyphes étroits, en forme

de trompette. Lichen commun; pousse à même la terre,

sur les tronçons d'arbre et à la base des arbres

Cladonia fimbriata 
28. Podétions sorédiês seulement dans le tiers supérieur; coupes larges, profondes. Lichen fréquent; pousse à même la terre et sur les tronçons d'arbre.. . . Cladonia conista

29. Scyphes dēformés, très irréguliers; podétions souvent fendus, courament recouverts de squamules (v. doublet 23). Plante commune; terricole........ (fig. 55) Cladonia phyllophora

29. Scyphes individualisés, de forme régulière; podétions couverts ou non d'un petit nombre de squamules . . . . . . . . . 30 .

30. Scyphes profonds, contenant de petites arêoles squameuses; podetions couverts d'un cortex en grande partie écaillé (décortiqué) laissant, par endroits, des plaques vertes sur un fond noir; squamules basilaires épaisses, convexes, au pourtour non lobé . . . . . . . . . . . 31. 30. Scyphes très peu profonds à plans, à prolifération habituellement marginale ou centrale; cortex en grande partie intact et lisse . . . . . . . . . . . . . . 32 .

31. Thalle vert-de-gris foncé à vert brunâtre; réaction nulle à $\mathrm{KOH}$; rebord des coupes exceptionnellement squamuleux. Espèce commune; colonise le sol nu et le roc . . . . . . Cladonia pyxidata

31. Thalle gris clair à gris brunâtre, KoH+ jaune (atranorine); pourtour des scyphes souvent squamuleux. Espèce rare; se développe sur les sols minces en pleine lumière . . Cladonia magyarica

32. Pođétion prolifère à partir du centre, produisant parfois quelques étages de scyphes. Espèce commune, hêliophile, s'installe sur la terre. . . . . . . . (fig. 56) Cladonia cervicornis subsp. verticillata (cladonie verticillée)

32. Lichen à prolifération marginale, donnant parfois naissance à plus d'un étage. Espèce commune, croissant sur un sol ensoleillé ou sur des touffes de mousses

33. Podétions sans sorédies ou granules; cortex KoH+ jaune pâle (atranorine) . . . . . . . . . . . . . . . . 34 .

33. Podêtions plus ou moins garnis de sorêdies ou de granules . . . . 40.

34. Podétions Pdt jaune vif (acide psoromique) . . . . . . . 35 . 34. Podétions Pd- ou Pdt rouge . . . . . . . . . . . 36. 
35. Grandes squamules basilaires étroites (4 à $6 \mathrm{~mm}$ de long par 2 à $3 \mathrm{~mm}$ de large), ascendantes et retroussées découvrant ainsi un revers blanc; cortex réparti en êpaisses plaques crevassées, souvent fendues ou perforées; petites apothécies communes, nombreuses sur chaque pođétion. Espèce rare; pousse sur les sols calcaires minces . . . . . . . Cladonia dahliana

35. Petites squamules disposées sur le podẻtion et à sa base, mesurant moins de 2 à $3 \mathrm{~mm}$ de long; podétions en grande partie décortiqués, garnis de petites plaques presque de la taille d'un granule, disséminées à la surface; podétions fendus et irréguliers, souvent ramifiês au bout; très peu d'apothécies. Taxon peu fréquent; terricole. . . Clađonia norrlinii

36. Podétions $1-1,5(2,5) \mathrm{mm}$ de hauteur, complètement dépourvus de cortex, se développant des marges de squamules ramifiés et finement lobés; thalle Pd+ rouge ( I'acide fumarprotocétrarique), KOH-. Espèce rare; s'installe sur l'écorce ou sur le bois. . . Cladonia caespiticia

36. Podétions d'au moins $2 \mathrm{~mm}$ de hauteur, partiellement cortiqués; se développant verticalement du niveau du substrat, habituellement des centres des squamules basilaires . . . . . . . . . . . . . . . . . 37.

37. Thalle $\mathrm{KOH}-$ (dépourvu de l'atranorine). Poussant ordinairement sur le bois, et de temps en temps, sur le sol. • • 38 .

37. Thalle (c.-à-d., l'envers des squamules) KOH+ jaune (atranorine). Lichen terricole... . . . . . . . . . . 39.

38. Thalle Pd+ rouge (l'acide fumarprotocetrarique); podétions 7-12 mm de hauteur, terminé par de très grandes apothécies brunes; squamules basilaires petites, épaisses, serrées. Lichen rare; s'installe sur la terre ou le bois... . . . . . . . . . . . Cladonia capitata

38. Thalle $\mathrm{Pd}-$; podétions $2-5 \mathrm{~mm}$ de hauteur, terminé par de grandes apothécies brunes jaunâtre ou brunes pâles; squamules basilaires miniscules, plus ou moins dispersées sur le substrat. Lichen rare; s'installe sur le bois, ou, rarement, sur le sol tourbeux . . Cladonia botrytes 
39. Très grandes squamules basilaires dressées, atteignant souvent plus de $6 \mathrm{~mm}$ de long; podétions vert foncé, irréguliers, souvent fendus longitudinalement, à surface lisse et uniforme; pas d'apothécies ordinairement. Plante rare; colonise le sol au soleil. . . . . . . . Cladonia turgida

39. Petites squamules basilaires, n'excédant pas $3 \mathrm{~mm}$ de long; podétions vert-de-gris, verruqueux, non squamuleux, souvent fendus ou "lacérés" dans le sens de la longueur, aux sommets couverts d'une ou de plusieurs grosses apothécies brunes. Lichen commun; terricole, vit dans les lieux ouverts . . Cladonia cariosa

40. Podêtions $\mathrm{KOH}+$ jaune vif, Pdt orange (acide thamnolique)

40. Podétions $\mathrm{KOH}-, \mathrm{KOH}+\mathrm{jaune}$ pâle, ou $\mathrm{KOH}+$ jaune brunâtre terne; Pd-, Pd+ jaune vif ou Pdt rouge . . . . . . . . . . . 42 .

41. Podétions et contour des squamules basilaires verruculeux; podétions immanquablement couverts de grosses apothécies brunes au bout. Taxon rare; pousse sur les tronçons d'arbre ou le sol. . . . . . . . . . . . . . . . Cladonia parasitica

41. Podẻtions pulvérulents (sorédies), dépourvus d'apothécies. plante peu fréquente; terricole, saxicole ou pousse sur les tronçons d'arbre . . . . . . . . . . . . Cladonia macilenta

42. Sorédies fines, pulvérulentes . . . . . . . . . . • • • . . 43.

42. Sorédies grossières, granuleuses; podétions effilés, Pd+ rouge ou $\mathrm{Pd}-$. . . . . . . . . . . . . . . . . 4 45.

43. Podêtions Pd-, très élancés, presque blancs garnis de sorédies d'aspect farineux; podétions quelquefois plus épais au bout (c.-à-d. "claviformes"); petites squamules basilaires au pourtour lobé ou finement découpé. Espèce commune; pousse sur les tronçons d'arbre, le sol ou la base des arbres . . . . . . . . . . . . . . . . . Cladonia bacillaris

43. Podétions Pdt rouge, habituellement olive à vert légèrement jaunâtre ou brunâtre (jamais blanc) . . . . . . . . . . . . • . . 44.

44. Podétions courts, trapus, ne dépassant pas, habituellement, $20 \mathrm{~mm}$ de haut s'amincissant régulièrement pour former une pointe; grosses squamules 
basilaires, au pourtour normalement non lobé ou pourvues de petits lobes non découpés, au centre desquelles s'élèvent les podétions; sorédies répandues généralement sur au moins $2 / 3$ du podétion. Lichen très commun; recherche différents milieux: la terre, ou le sol sur roc, les tronçons d'arbre et la base des arbres ( $v$. doublet 26) . . . . . . . (fig. 57) Cladonia coniocraea

44. Hauteur des podétions excédant généralement $30 \mathrm{~mm}$; petites squamules basilaires au pourtour découpé et lobé; soralies disposées surtout dans la moitié supérieure des pođétions. Taxon rare; pousse sur les tronçons d'arbre et le sol . . . . . . . . Cladonia cornuta

45. Podétions pdt jaune, KoHt jaune pâle (acide psoromique et atranorine) (v. doublet 35) . . . . . . . . Cladonia norrlinii

45. Podétions Pd- ou Pd+ rouge, KOH+ plus ou moins brunâtre (pas d'atranorine) . . . . . . . . . . . . . . . . . 46.

46. Podétions cylindriques, n'atteingnant pas $5 \mathrm{~mm}$ de hauteur, verruculeux (sorédies) à la base, devenant presque farineux aux extrémitês; squamules basilaires divisées et lobées, parfois sorédiées; réaction rouge à Pd (acides fumarprotocétrarique et grayanique). Plante peu fréquente; s'installe sur le bois et au pied des arbres . . . . . . . . . . . . cladonia cylindrica 46. Podêtions de plus de $5 \mathrm{~mm}$ de haut, quelque peu irréguliers à ramifiés au sommet . . . . . . . . . . 47.

47. Podétions atteignant souvent plus de $30 \mathrm{~mm}$ de haut, ramifiés aux extrêmités, avec les aisselles perforées, habituellement squamuleux; réaction rouge à Pd. Espèce terricole ou pousse sur les tronçons d'arbre . . . . . . . . cladonia scabriuscula

47. Hauteur des podétions n'atteignant généralement pas $30 \mathrm{~mm}$; structures non rameuses ou prolifères aux extrémités, mais dépourvues d'aisselles perforées. . . . . . . . . . . . 48 .

48. Podêtions élancés, avec des surfaces décortiquées, opaques; Pd-, ou Pdt rouge. Plante commune (voir

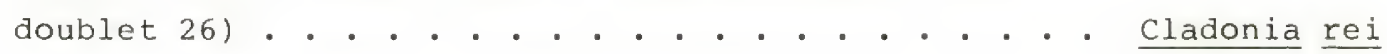


48. Podétions trapus, très irréguliers; superficies decortiquees, translucides (pellucides); Pdt rouge. Taxon rare; se développe sur du bois pourri.. Cladonia anomaea

49. Branches ou tiges à croissance verticale dirigée vert le haut, naissant souvent d'une croûte basilaire, granuleuse, ou poussiéreuse . . . . . . . . . . . . . . . . 50 .

49. Branches ou tiges poussant perpendiculairement à une surface verticale, ou pendantes, ne provenant jamais d'une croûte basilaire. . . . . . . . . . . . . . . 53 .

50. Tiges très courtes (jamais plus de $12 \mathrm{~mm}$ de haut), simples, nues, croissant sur une croûte basilaire verruculeuse ou aréolée et blanc verdâtre, devenant pulvêrulente par endroits, se terminant par une fructification brun pâle (apothécie); $\mathrm{KOH}+$ jaune, $\mathrm{Pd}+$ orange (acide stictique). Lichen rare; vit sur les rochers ombragés et la terre... . . (fig. 60) Baeomyces rufus (N.B.: Une autre espèce de Baeomyces, B. roseus, bien qu'elle soit commune à partir de Gaspé en direction de l'est, n'a été récoltée qu'à une seule reprise dans la région: au nord d'ottawa, dans le parc de la Vérendrye. Son thalle est blanc, continu à verruculeux, et il deveint jaune au contact de Pd et de $\mathrm{KOH}$ (acide baeomycésique); il est pourvu de podétions courts qui se terminent par de très grandes apothécies roses.

L'espèce vit sur des sols perturbés, souvent sableux, et fréquemment le long des routes.)

50. Branches fortement rameuses, plus ou moins ascendantes ou étendues tel un coussin appliqué au support; tiges de plus de $25 \mathrm{~mm}$ de haut, recouvertes de lobes semblables à des granules ou des écailles; KoH+ jaune . . . . . . . . 51.

51. Pas de céphalodies; écailles sur le "podétion" (phylloclades)

larges et lobées; "pođétions" généralement plus ou moins plats près du support et à faces supérieure et inférieure nettement distinguables; thalle Pd- ou Pd+ jaune pâle (atranorine). Taxon très commun; croît directement sur les roches non calcaires, ordinairement dans des lieux très ensoleillés......... (fig. 59) stereocaulon saxatile 
51. Présence de céphalodies; phylloclades élargies ou finement découpées; "podétions" surtout dressés, dépourvus de faces supérieure et inférieure distinctes; thalle pd+ orange pâle (acide stictique). Plante rare; normalement terricole... . . 52.

52. Grandes céphalodies gris bleuté, en forme de tumeur, très visibles sur les "podétions"; phylloclades finement découpées . . . . . . . . . Stereocaulon dactylophyllum

52. Petites céphalodies noires, enfouies dans un épais tomentum duveteux et gris à la surface du "podétion"; épaisses phylloclades au contour lobé, mais non finement découpé . . . . . . . . . . . . Stereocaulon tomentosum

53. Une coupe transversale révèle la rondeur presque parfaite des rameaux; branches plus ou moins filamenteuses . . . . . . . . . 54 .

53. Une coupe transversale présente des rameaux nettement aplanis ou très irréguliers, au moins à la base. . . . . . . . . . 58.

54. Thalle vert jaunâtre; filaments pourvus au centre d'un axe chondroïde et élastique (fig. 67) . . . . . . . . 55.

54. Thalle brun à presque noir; filaments depourvus, au centre, d'axe chondroïde (fig. 62) . . . . . . . . . 57.

55. Court thalle buissonnant, ni long, ni pendant... . . . . . 56 .

55. Thalle nettement pendant, dont les principales branches sont, de façon générale, plus foncées que les ramifications secondaires; thalle isidié. Taxon rare; épiphyte. . Usnea filipendula

56. Filaments à peine "bosselés" et anguleux, particulièrement près de la base (fig. 6lb); surface des branches principales sans miniscules saillies (papilles); isidies formant un revêtement serré au jeunes branches; pas de sorédies. Lichen peu fréquent, épiphyte............. (. . (fig. 6l) Usnea hirta

56. Eilaments jamais bosseles ou anguleux, dont les branches principales au moins sont garnies de papilles et dont les jeunes branches sont souvent dotées de sorédies mêlêes d'isidies. Plante fréquente, épiphyte 
(59)
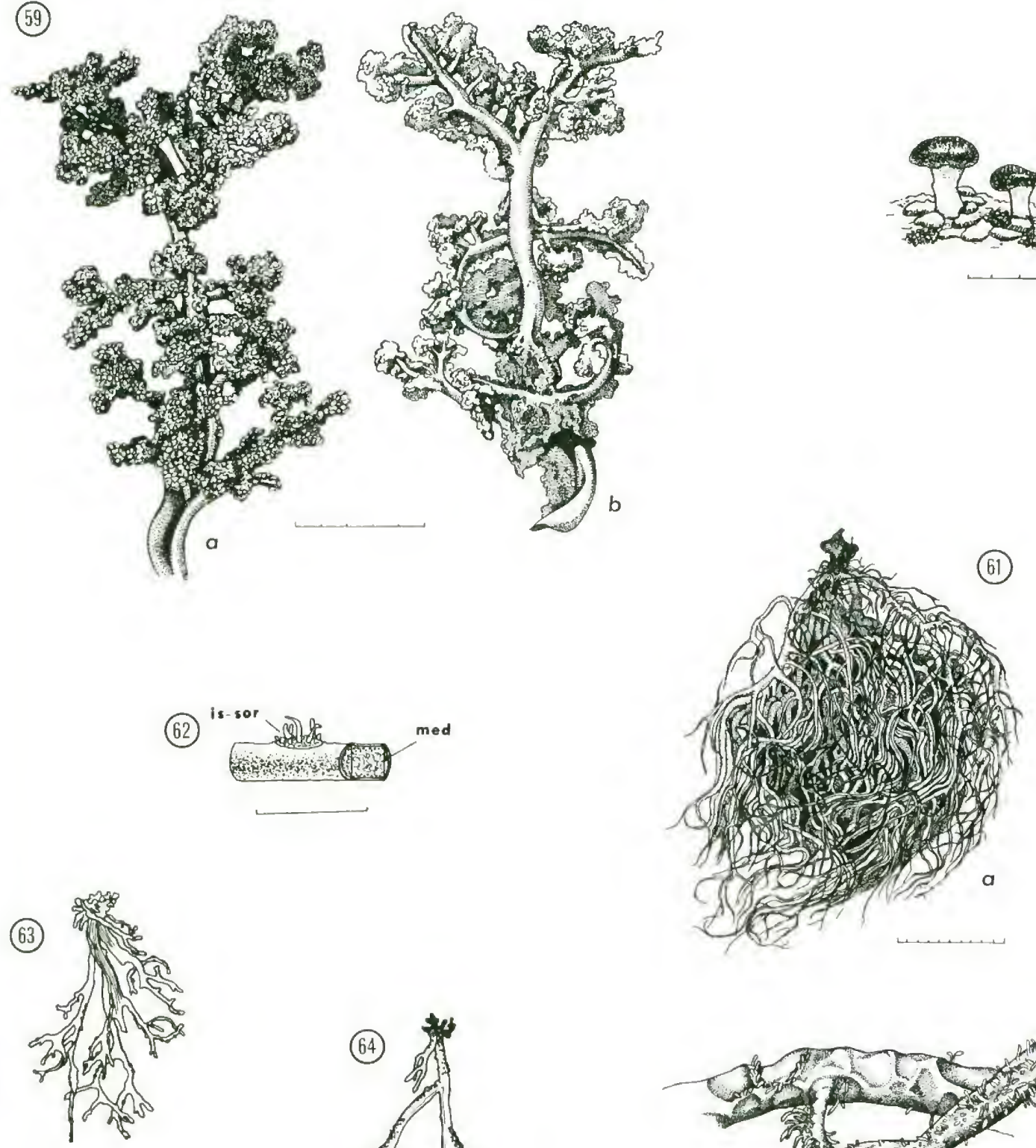

(62) is-sor
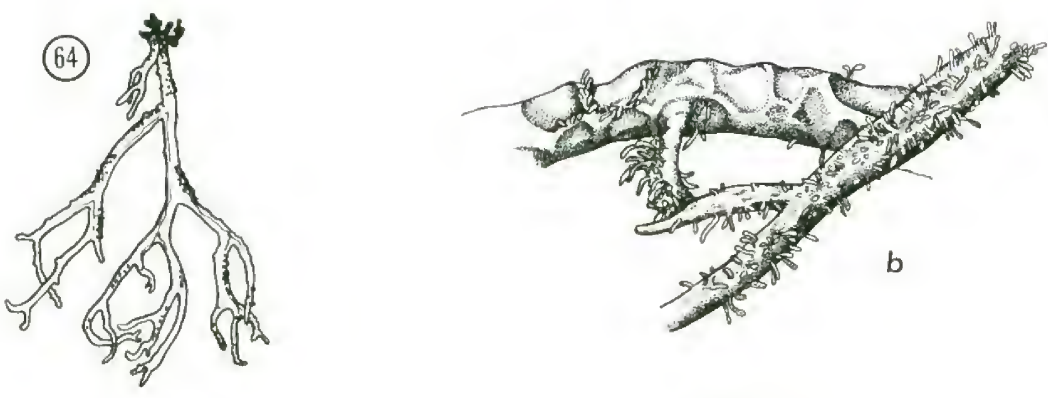

Figures 59 à 64.-59. Stereocaulon saxatile: a) face "supérieure"; b) face "inférieure".-60. Baeomyces rufus.-61. Usnea hirta: a) thalle;

b) rameaux à sections plus ou moins irrégulièrement anguleuses et isidiés.-62. Bryoria furcelzata, fragment de rameau: médulle uniforme et soralie isidiée.-63. Ramalina intermedia.-64.

Evernia mesomorpha. Échelle: chaque division $=1 \mathrm{~mm}$. 
57. Thalle buisonnant, irrégulièrement ramifié, presque aussi large que long; branches recouvertes, par endroits, de plaques de sorédies elliptiques mêlées d'isidies spinuliformes, menues mais remarquables (fig. 62); soralies et thalle Pd+ rouge. Espèce fréquente; crô̂t surtout sur les

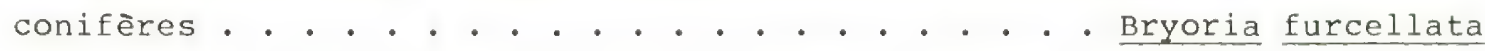

57. Thalle plus ou moins pendant, plus long que large, aux ramifications régulières ou irrégulières, entièrement dépourvu de sorédies ou d'isidies; médulle Pd+ rouge (réaction parfois difficile à déceler). Taxon rare; crô̂t sur les conifères. . . . . . . Bryoria trichodes subsp. trichodes

58. Rameaux anguleux et inégaux, s'aplatissant quelquefois; thalle très doux et flexible; surface des branches mate, grossière, parsemée de sorédies granuleuses sur la plus grande partie de la surface. Lichen fréquent; épiphyte

(fig. 64) Evernia mesomorpha

58. Rameaux assez rigides, clairement aplatis, du moins à la base; branches luisantes et lisses (quoique souvent striées); sorédies présentes ou absentes; si elles sont présentes, elles se disposent en structures ou plaques distinctes . . . . . . . . . . . . . . . 59.

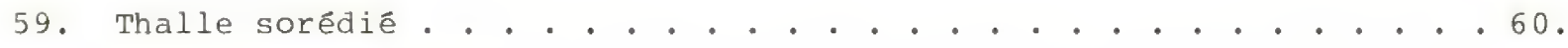

59. Thalle non sorédié . . . . . . . . . . . . . . . . . 61.

60. Rameaux relativement fins, généralement de moins de 1,5 mm de large, s'élargissant souvent; sorédies disposées en plaques remarquables et elliptiques le long de la marge des branches; très peu de fructifications. Lichen peu fréquent; pousse sur les parois rocheuses, rarement sur l'écorce. . . . . . . . (fig. 63) Ramalina intermedia

60. Branches ordinairement trapues, de 1,5 à $4 \mathrm{~mm}$ de large; soralies en grosses masses souvent cucullées, surtout à l'extrémité des rameaux. Plante rare, saxicole ou corticole. . . . . . . . . . . . . . Ramalina obtusata

61. Branches larges, pleines, dont la longueur va jusqu'à $3 \mathrm{~mm}$, quelquefois davantage, entièrement aplaties; grosses apothécies jaunes et discoïdes, communes au bord des branches 
ou près de leur extrémité. Taxon fréquent, épiphyte

. . . . . . . . . . . . . . . . Ramalina americana

61. Branches étroites, de moins de $3 \mathrm{~mm}$ de large, plans seulement

à la base, un peu creux et cylindriques (fistuleux), de même

que perforés d'orifices ou de trous. Lichen rare, épiphyte

Ramalina dilacerata 


\section{RÉFÉRENCES}

*Anonym 1974. Bulletin de terminologie, Secrétariat d'État, no 156, Ottawa. $430 \mathrm{p}$.

Bennett, J. 1979. Lichens: the first mercy of the earth. Harrowsmith $4: 62-65,69+$.

Brodo, I.M. 1967a. Lichens of the Ottawa area. I. Trail \& Landscape $1: 40-45$.

1967b. Lichens of the Ottawa area. II. The fruticose species. Trail \& Landscape 1:114-115, 118-121.

1968. The Iichens of Long Island, New York: A

vegetational and floristic analysis. N.Y. State Mus. Sci. Serv. Bull. $410: 1-330$.

1972. Lichens of the Ottawa area. III. The crustose species. Trail \& Landscape 6:15-26.

*Fouchier, J.; Billet, F. 1961. Dictionnaire de chimie, Netherlands University Press N.V., Amsterdam. 429 p.

Hale, M.E. 1979. How to know the lichens. 2d ed. Wm. Brown Co., Dubuque, Iowa. $246 \mathrm{pp}$.

Harris, R.C. 1973. The corticolous pyrenolichens of the Great Lakes Region. Michigan Bot. 12: 3-68. 1977. Lichens of the Straits Counties, Michigan. Publ. by author, Ann Arbor, Michigan. (1itho.) 150 pp.

Macoun, J. 1898. The cryptogamic flora of Ottawa. Ottawa Naturalist $12: 25-32 ; 12: 49-56 ; 12: 93-100$.

1902. The cryptogamic flora of Ottawa. Ottawa Naturalist

$20: 177-186$.

MCMillan, B. 1979. Nature's perfect partnership, or, how a lowly fungus finds happiness with a simple alga. Nature Can. 8:2l-26.

*Ozenda, P.; Clauzade, G. 1970. Les lichens. Étude biologique et flore illustrée. Masson et Cie, Paris. 801 p.

*Souchon, C. 1971. Les lichens. Presses universitaires de France, Paris. $124 \mathrm{p}$.

Tibell, L. 1975. The Caliciales of boreal North America. Symb. Bot. Upsal. $21(2): 1-128+$ viii.

*Ouvrages spécialisés consultés pour la traduction. 


\section{GLOSSAIRE}

Aciculaire (adj). En forme d'aiguille, c.-à-d. mince et pointu aux deux extrémités.

Amphithécium ( $\mathrm{n} \mathrm{m}$ ). Partie de l'apothécie lécanorine qui entoure le rebord propre (fig. $70 \mathrm{~b}$ ) et qui contient ordinairement des algues; bord thallin.

Apothécie (n f). Ascocarpe en forme de disque ou de coupe (fig. 65 et 70 ). Aréolé (adj). Divisé en petits compartiments irréguliers, habituellement polygonaux (aréoles), un peu comme le carrelage d'un plancher (fig. I, $2 a$ et 68 ).

Ascocarpe $(\mathrm{n} \mathrm{m})$. Fructification chez les Ascomycètes; organe qui porte les asques, ces derniers contenant les ascospores.

Ascohyménial (adj). Se dit d'une sorte d'ascocarpe qui possède de vêritables paraphyses et dont les asques ont une seule assise; caractéristique de la sous-classe des Ascomycetidae.

Ascoloculaire (adj). Se dit d'une sorte d'ascocarpe dont les asques (dont les parois sont constituées ordinairement de quelques assises) prennent naissance dâns un tissu mycêlien homogène et sont dissêminés à maturité, non pas à travers de véritables paraphyses, mais à travers des pseudo-paraphyses; caractéristique des membres de la sous-classe des Loculoascomycetidae.

Ascospore ( $\mathrm{n}$ f). Spore qui prend naissance dans un asque (fig. 72 ). Asque $(\mathrm{n} \mathrm{m})$. Organe vésiculaire chez les Ascomycètes, où se forment les ascospores (fig. 71 ).

Bisérié $(a d j)$. Se dit de spores disposées en deux files à l'intérieur de l'asque (fig. $71 \mathrm{a}$ ).

Bord thallin $(\mathrm{n} \mathrm{m})$. V. amphithécium.

Calcaire $(\mathrm{n} \mathrm{m})$. Roche composée de carbonate de calcium et produisant une effervescence marquée $\left(\mathrm{CO}_{2}\right)$ au contact d'un acide fort.

Carbonacé (adj). Noir, opaque et habituellement friable; en règle générale, il est impossible de distinguer les cellules les unes des autres dans un tissu.

Céphalodie ( $\mathrm{n}$ f). Sorte de petite excroissance en forme d'une galle à l'intérieur ou à l'extérieur de certains lichens; elle contient des algues bleues.

Chlorococcale ( $\mathrm{f} f$ ). Algue verte unicellulaire dont les cellules sont sphériques.

Cil $(\mathrm{n} \mathrm{m})$. Appendice filiforme qui est situé à la marge du thalle ou des apothécies chez bon nombre de lichens foliacés et fruticuleux (fig. $46 b)$. 
Cloison (n m). V. septum.

Continu (adj). Se dit d'un thalle uniforme ou à peine fendillé (fig. 69).

Coralloïde $(\operatorname{adj})$. a) Qui possède ou qui est composé d'excroissances cylindriques, finement ramifiées. b) se dit de la forme de certaines isidies.

Cortex $(n \mathrm{~m})$. Couche protectrice du thalle ou de l'apothécie; entièrement composée d'hyphes; d'apparence souvent "celluleuse"

(paraplectenchymeux), fig. 65 .

Cortex de l'amphithécium $(\mathrm{n} \mathrm{m})$. Couche protectrice de l'amphithécium.

Cortiqué (ajd). Pourvu d'un cortex.

Corticole (adj). Qui pousse sur l'écorce.

Couche algale ( $\mathrm{n} f)$. Couche de cellules algales dans le thalle d'un lichen (fig. 65); couche "gonidiale" de la litérature lichênologique de l'Europe.

Crénelé (adj). Dont la marge est découpée en dents arrondies ou en lobes minuscules.

Crampon ombilical ( $\mathrm{n} \mathrm{m}$ ). Organe fixateur, court, épais, en forme de tige et constitué uniquement d'hyphes chez différents lichens foliacés ou presque foliacés, surtout chez les Umbilicaria.

crustacé $(a d j)$. S'applique à une sorte de thalle qui adhère entièrement au substrat et dont le cortex inférieur fait défaut; il ne peut pas être détaché intact sans que l'on prélève également un morceau du substrat (fig. 73).

Décortiqué $(\operatorname{adj})$. Se dit d'un thalle dont le cortex s'est écaillé ou décomposé.

Dichotome (adj). Qui se ramifie en deux parties égales, comme dans la lettre "Y" (p. ex. fig. 50).

Dorsi-ventral (adj). Pourvu d'une face supérieure et d'une face inférieure. Endolithique (adj). Se dit d'un thalle qui "crô̂t" dans la roche, en s'infiltrant entre les cristaux de la roche; le thalle est souvent invisible ou à peine visible.

Épispore (n f). Enveloppe gêlatineuse et transparente, d'épaisseur irrégulière bien souvent, qui enveloppe les ascospores de grand nombre de lichens; souvent appelée "halo" (fig. 72a).

Épithécium ( $n \mathrm{~m}$ ). Partie supérieure de l'hyménium, formée de l'extrémité épaissie des paraphyses; d'ordinaire pigmentée et contenant parfois quelques granules (fig. 70 ).

Érugineux $(a d j)$. Vert bleuâtre.

Érumpent (adj). Qui émerge de la surface du thalle, en écartant les tissus. 
Étage ( $n f$ ). Évasement du podétion en forme de disque chez quelques espèces de Cladonia (p. ex. C. cervicornis), où prennant naissance une

ou plusieurs branches (fig. 56).

Fendillé (adj). Strié de fentes minuscules (p. ex. fig. 26).

Foliacé (adj). Se dit d'un thalle plus ou moins "feuillu", nettement dorsi-ventral, qui adhère au substrat en un seul point (ombiliqué) ou en plusieurs points, parfois même presque totalement (fig. 31 - 52).

Fruticuleux (adj). Se dit d'un thalle en forme de tige, pendant ou buissonneux dont normalement il est difficile de distinguer la face inférieure de la face supérieure (fig. 53 - 64).

Fusiforme (adj). Étroit, qui s'amincit au bout, normalement pointue aux deux extrémités; en forme de fuseau ou de cigare.

Glabre (adj). a) Dont la surface est plus ou moins lisse et reluisante. b) Dépourvu de tomentum.

Gloeocapsa $(\mathrm{n} \mathrm{m})$. Algue bleue constituée de petites colonies de cellules sphériques ( 2 - 8) emprisonnées dans une épaisse enveloppe gélatineuse, chaque cellule étant elle-même enveloppée d'une gaine gélatineuse.

Granule ( $(\mathrm{f})$. Particule cortiquée, sphérique ou presque ronde.

Granuleux $(a d j)$. a) Qui possède des granules ou des particules semblables à des granules. b) S'applique aux sorédies composées de particules assez grosses pour être facilement observées sous un microscope stéréoscopique, donc grossières et non pas pulvérulentes comme les sorédies farineuses.

Halo $(\mathrm{n} \mathrm{m})$. V. épispore. Hyalin $(\operatorname{adj})$. Incolore.

Hyménium $(n \mathrm{~m})$. Tissu fertile de l'ascocarpe fait d'asques et de paraphyses ou de pseudo-paraphyses (fig. 70).

Hypophléode (adj). Se dit des lichens corticoles qui poussent en grande partie ou entièrement sous une ou plusieurs couches de l'écorce.

Hypothalle $(\mathrm{n} \mathrm{m})$. Partie inférieure du thalle qui ne joue aucun rôle dans la photosynthèse, qui borde la marge de bien des espèces crustacées d'un liséré blanc ou pigmenté, et qui souvent forme un bourrelet entre les aréoles ou les granules chez d'autres espèces crustacées (fig. 68); protothalle.

Hypothécium $(\mathrm{n} \mathrm{m})$. Tissu situé directement sous l'hyménium et au-dessus du rebord propre (fig. 70a); souvent difficile à differencier du rebord propre.

Imbriqué (adj). Se dit des écailles ou des squamules qui se chevauchent à la manière des bardeaux d'un toit (p. ex. fig. 21). 
Involucrellum $(\mathrm{n} \mathrm{m})$. Sorte de couvercle qui coiffe le pyrénium de bon nombre de périthèces à la surface des lichens; ordinairement carbonacé (fig. $26)$.

Involuté $(a d j)$. Dont la marge est roulée vers l'intérieur.

Isidie ( $\mathrm{f}$ ). Minuscule excroissance à la surface du thalle, cylindrique ou coralloïde, cortiquée et contenant des algues; elle servirait à la multiplication végétative (propagule) (fig. 65).

Isthme $(\mathrm{n} \mathrm{m})$. Canal étroit reliant les deux logettes d'une spore polariloculaire (fig. 72d); s'applique à la distance séparant les deux logettes.

Labriforme (adj). a) En forme de lèvre. b) S'applique aux soralies; la lèvre est gênéralement formée de la marge révolutée d'un thalle ou de l'extrêmitê relevêe d'un lobe creux, sorédiêe sur la face inférieure (c.-à-d. exposée), comme chez Hypogymnia physodes (fig. 42).

Lécanorin (adj). Se dit d'un type d'apothécie pourvue d'un amphithécium qui contient habituellement des algues, comme dans le genre Lecanora (fig. $70 \mathrm{~b}$ ).

Lécidéin $(a d j)$. Se dit d'un type d'apothécie dépourvue d'amphithécium. et où le parathécium forme par conséquent le bord de l'apothécie (c.-à-d. le rebord propre), comme dans le genre Lecidea (fig. 70a).

Lépreux (adj). Presque entièrement composée de granules plus ou moins agglomérées, appelées sorédies.

Lignicole (adj). Qui pousse sur le bois nu (l'aubier), comme les tronçons d'arbre écorcés ou les clôtures de bois.

Lobule $(n \mathrm{~m})$. Petit lobe souvent en forme d'écaille, qui pousse sur certains thalles foliacés, soit à leur surface, soit le long de la marge, ou encore parfois au bord des apothécies; d'ordinaire concolore au thalle, le lobule présente généralement les mêmes caractères que le thalle lui-même (fig. 65).

Logette ( $\mathrm{n} f$ ). Cavité cellulaire de l'ascospore.

Mal dêlimitê (adj). Se dit d'un thalle dont le pourtour se distingue difficilement.

Mazêdium $(\mathrm{n} \mathrm{m})$. Masse d'ascospores et de paraphyses provenant de la désintégration des asques chez un certain type d'ascocarpe, comme chez les Chaenotheca.

Médulle ( $\mathrm{n}$ f). Partie à l'intérieur du thalle ou d'une apothécie lécanorine qui se compose ordinairement d'hyphes lâches (fig. 65).

Mural (adj). Se dit des spores à septum logitudinal et transversal, disposées un peu comme les briques d'un mur (fig. $72 a, b$ ). 
Nostoc $(\mathrm{n} \mathrm{m})$. Genre d'algues bleues vivant chez un grand nombre de lichens; libres, elles forment des chapelets ou des filaments de cellules mais, lichénisées, elles vivent seules ou en colonie de quelques cellules (fig. 33 et 35 ).

Ombiliqué (adj). Fixé par un seul point central (le crampon ombilical) situé sur la face inférieure du thalle.

Ostiole $(\mathrm{n} \mathrm{m})$. Pore apicale, petit et rond dans diverses sortes d'ascocarpes (fig. 74).

Paraphyse ( $\mathrm{f}$ f). Hyphe stérile, parfois ramifié; ces hyphes sont dispersés dans l'hyménium parmi les asques chez un membre des Ascomycetidae.

Paraplectenchymateux (adj). S'applique au tissu mycélien à qui les cellules courtes et les hyphes fortement ramifiês et orientés dans toutes les directions confèrent un aspect celluleux en coupe.

Pelté (adj). Fixé au centre de la face inférieure; en forme de parapluie. Périthèce ( $(\mathrm{m})$. Ascocarpe en forme de sphérule (fig. 69, 74c, 26, 27); sessile ou, plus souvent, enfoncé partiellement ou entièrement dans le thalle.

Phycosymbionte $(\mathrm{n} \mathrm{m}$ ). Algue (symbionte) du thalle lichénique. Phylloclade ( $\mathrm{f}$ f). Minuscule excroissance souvent lobée ou en forme d'écaille, située sur les rameaux de certains membres du genre Stereocauton (fig. 59).

placodiomorphe (adj). Se dit d'un thalle crustacé épais, dont le pourtour est lobé (p. ex. fig. 22).

plié longitudinalement (adj). Strié de crêtes ou de sillons plus ou moins longitudinaux.

Podétion $(\mathrm{n} \mathrm{m})$. Tige qui prolonge l'apothécie (qui se forme ordinairement à partir de l'hypothécium et du stipe); dans un second temps, une couche algale et un cortex s'installent généralement dans cette tige (comme chez les Cladonia) et cette dernière peut être courte mais non ramifiée, ou relativement longue et fortement branchue (fig. 53 - 58 , $66)$.

Polariloculaire (adj). Se dit des spores qui possèdent deux cavitēs sẻparées par un septum relativement épais, percé d'un canal etroit ou isthme (fig. 72d, 14, 15); caractéristique des membres des Teloschistaceae. Pruineux (adj). Qui a l'apparence du givre (ordinairement blanc ou gris). Pseudocyphelle ( $\mathrm{f} f$ ). Minuscule point blanc ou pore qui se trouve en grand nombre sur la face supérieure et parfois sur la face inférieure de bien des espèces foliacées; ce pore se forme à la suite de la rupture du cortex et de l'affleurement des hyphes de la médulle (fig. 65). 
Pseudo-paraphyses ( $\mathrm{f}, \mathrm{pl}$ ). Restes du tissu mycélien qui se mêlent aux asques dans l'ascocarpe ascoloculaire; ils sont souvent très ramifié et anastomosés.

Pulvérulent $(\operatorname{adj})$. A l'état de pouđre.

Pycnide ( $n$ f). Organe globuleux ou urcéolé, ordinairement de très petite taille, où se forment les spores ou conidies; vu de l'extérieur, il ressemble à s'y méprendre à un périthèce (fig. 5lb).

Pyrénium $(\mathrm{n} \mathrm{m})$. Dans cette clef, il s'agit de l'assise qui entoure le périthèce (v. fig. 26: l'assise pâle; fig. 74c: l'assise noire).

Rebord propre $(\mathrm{n} \mathrm{m})$. partie de l'apothécie située sous l'hypothécium, qui forme le bord de l'apothécie lécidéine (fig. 70a) et est entourée de l'amphithécium dans l'apothécie lécanorine (fig. 70b); "excipulum proprium" .

Relâché (adj). Lâche; qui n'est pas compact.

Renflé $(\operatorname{adj})$. Gonflé et creux.

Révoluté (adj). Se dit d'une marge enroulée vers l'extêrieur (p. ex. fig. 38).

Rhizine ( $\mathrm{n} f$ ). Prolongement du cortex inférieur, uniquement constitué d'hyphes, qui sert gênéralement à fixer le thalle foliacé au substrat; sa longueur, son épaisseur, sa couleur et son degré de ramification sont variables (fig. 65).

Saxicole (adj). Qui pousse à même le roc, la pierre, les cailloux, le béton ou la brique.

Scabre $(\operatorname{adj})$. Dont la surface est légèrement rude, presque croûteuse.

Scyphe $(\mathrm{n} \mathrm{m})$. Évasement du podetion en forme de coupe ou de trompette chez quelques espèces de Cladonia (p. ex. C. chlorophaea; fig. 58).

Septum $(n \mathrm{~m})$. Cloison; paroi transversale des hyphes et des spores. Sessile $(a d j)$. Dépourvu de pied. Simple $(a d j)$. Non ramifié; non cloisonné.

Soralie ( $\mathrm{n} f)$. Organe ou partie du thalle où se forment les sorédies; sa forme est très variable (fig. 65).

Sorédie ( $\mathrm{f}$ f). Organe servant à la multiplication végétative, constitué de quelques cellules algales et d'hyphes entrelacés et enveloppés d'une couche d'hyphes; totalement dépourvu de cortex; les sorédies prennent naissance en groupes bien localisés, appelés soralies, ou s'étalent sur des sections importantes du thalle.

Sorédies farineuses ( $\mathrm{f}$ f, pl). Sorédies très fines, pulvérulentes.

Spore ( $\mathrm{n}$ f). Organe de reproduction unicellulaire ou pluricellulaire, susceptible de donner naissance à une nouvelle plante; ce terme est ici synonyme d'ascospore. 
Squamule $(n \mathrm{f})$. Lobe ou aréole de petite taille et en forme d'écaille, au moins partiellement ascendant en règle général (p. ex. fig. 21).

Squamules basilaires ( $\mathrm{f} f, \mathrm{pl}$ ). Petits lobes en forme d'écaille, qui forment le thalle primaire des Cladonia (fig. 66).

Squarreux (adj). Pourvu de rameaux courts, raides et perpendiculaires; semblable à un rince-bouteilles (fig. 65).

ococcus $(n \mathrm{~m})$. Petite algue verte unicellulaire dont les cellules sont courtes et cylindriques (en forme de bâtonnet).

Sub- (préfixe). a) En partie. b) De façon incomplète. c) A peu près. d) Sous.

Superficiel (adj). Situé sur la face supérieure du thalle.

Terminal (adj). Situé à l'extrémité d'un lobe ou d'une branche; s'applique généralement aux soralies (p. ex. fig. 47).

Terricole (adj). Qui pousse sur le sol ou sur le sable.

Thalle $(n \mathrm{~m})$. Appareil végétatif des lichens composé d'une algue et d'un champignon.

Thalle primaire ( $\mathrm{n}$ ). Base du thalle des Cladonia, composée d'écailles

en forme de feuilles, appelés squamules.

Thallin (adj). Se dit du thalle d'un lichen; semblable à un thalle par

l'aspect extérieur ou par la structure.

Tomenteux (adj). D'aspect duveteux ou laineux.

Tomentum $(\mathrm{n} \mathrm{m})$. "Pubescence" fine ou duvet.

Trebouxia $(\mathrm{n} \mathrm{m})$. Genre d'algues vertes unicellulaires. Un chloroplaste discoïde, au pourtour lobé ou crénelé, occupe presque toute la place dans la cellule. Il s'agit du phycosymbionte le plus commun chez les lichens. On utilise ici ce terme dans son ancienne acception, en incluant Pseudotrebouxia.

Trentepohlia $(\mathrm{n} \mathrm{m})$. Genre d'algues vertes filamenteuses, qui vit dans bon nombre de lichens crustacês; lichênisée, l'algue forme souvent de courtes chaînettes ou est unicellulaire. Les globules, qui contiennent un pigment rouge orangé, sont communs chez les algues libres, mais sont moins fréquents ou absents chez les algues lichénisées. Unisếrié (adj). Se dit des spores disposées en une seule file dans l'asque. Veine ( $\mathrm{n} f$ ). Crêtes large ou étroites, souvent pigmentées et situées sur la face inférieure de certaines espèces de Peltigera (fig. 38).

Vermiforme (adj). Qui a la forme d'un ver, c.-à-d., allongé, courbé et plus ou moins rond.

Verrue ( $\mathrm{n} f)$. Excroissance thalline, bien évidente (p. ex. fig. 24).

Verruqueux $(a d j)$. Couvert de protuberrances arrondies, en forme de verrue ( $p$. ex. fig. 7).

Verruculeux $(\operatorname{adj})$. Finement verruqueux. 
Figures 65 à 74: Caractères morphologiques des lichens. -65. Représentation semi-schêmatique d'un thalle foliacé: al, couche algale; apo, apothécie a) comme chez Umbilicaria, b) comme chez Parmelia, Physcia, etc.; $\underline{\text { is, }}$ isidies; $\underline{l c}$, cortex inférieur; lob, lobules; med, médulle; ps, pseudo-cyphelles; rh, rhizines a) simples, b) en touffe comme chez Peltigera, c) dichotomes, d) squarreuses; sor, soralies; uc, cortex supérieur. -66. Podétion de Cladonia cristatella: apo, apothécium; bsq, squamules basilaires; pod, podétion; sq, squamules du podetion. -67. Usnea subfloridana a) jeune thalle, b) section d'un rameau: cor, cortex, med, médulle, st, axe chondroïde central. Figures 68 à 74 : lichens crustacés. -68. Thalle aréolé vu de dessus et de côté: ar, aréole; apo, apothécie; pth, hypothalle; sub, substrat. -69. Thalle continu, lisse dans lequel s'enfoncent les périthèces (per). -70 . Apothécies. a - b: en coupe transversale; c - d: vues de l'extérieur. a) et c) type lécidéin, b) et d), type lécanorin: al, algues; amph, amphithécium; epi, épithécium; exc, rebord propre; hym, hyménium; hyp, hypothécium. -71. Asques qui contiennent des spores, a) type lécanorin et type lécidéin, b) Sarcogyne (asque myriosporé), c)

Pertusaria. -72. Ascospores: a) murales, entourées d'un "halo" gélatineux et transparent chez Rhizocarpon lavatum; b) murales, dépourvues de halo chez Polyblastiopsis; c) simples (unicellulaires ou sans cloison) chez Lecanora; d) polariloculaires chez Caloplaca; e) en forme de rein (légèrement courbées), à une cloison chez Lecania dimersa; f) filiforme chez Conotrema; g) fusiforme, dont les cellules sont cylindriques, chez opegrapha pulicaris; h) fusiformes, dont les cellules sont lenticulaires, chez Graphis scripta; chez Mycocalicium; j) chez Calicium; k) à une cloison, dont les cellules sont de dimensions variables chez Catizlaria laureri; 1) à une cloison, dont l'épaisseur des parois est égale, chez Buelıia; m) à une cloison, dont l'épaisseur des parois est inégale, chez Rinodina; n) parois épaisses et striées radialement des spores de Pertusaria macounii. -73. Thalle crustacé vu en coupe: al, algue; cor, cortex; med, médulle; sub, substrat. -74 . Fructification (ascocarpes) vues en coupe: a) biatorines, Lecidelza stigmatea; b) Pertusaria macounii; "ost", ouverture dans l'apothécie semblable à un ostiole; c) périthèce chez Pyrenula: ost, ostiole; d) Diploschistes scruposus. 


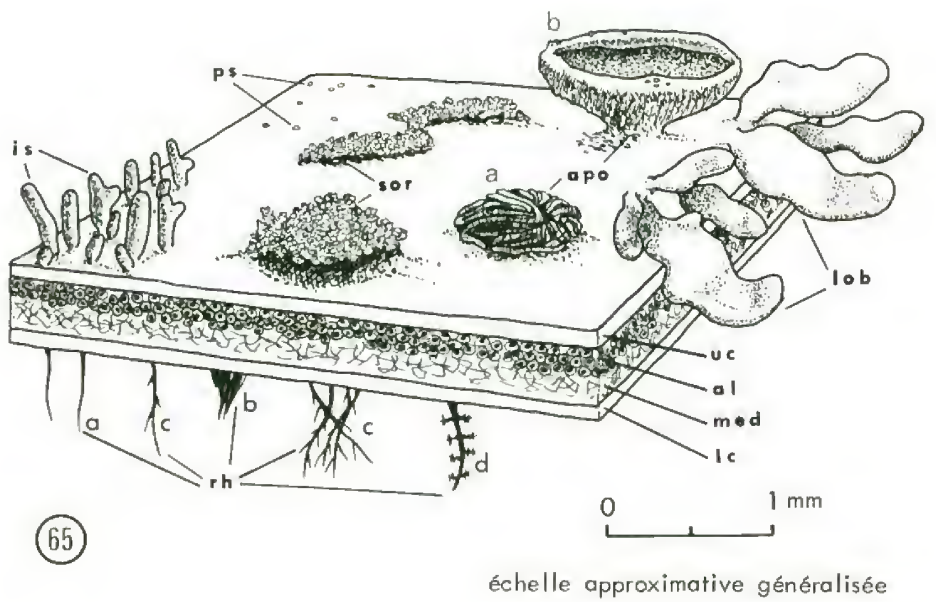

(66)

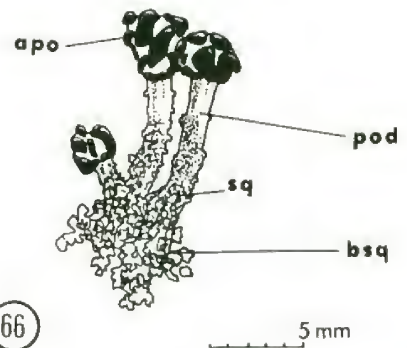

échelle approximative généralisée

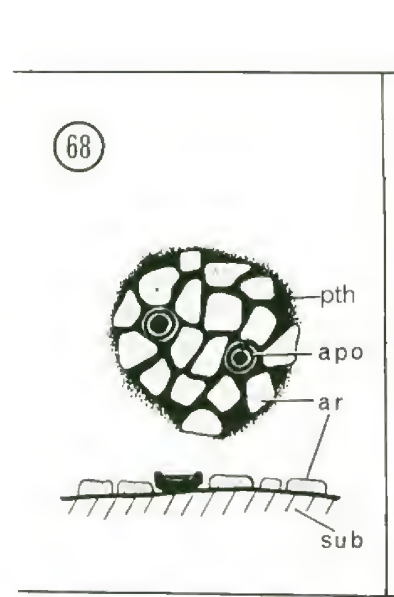

(69)

(70)

(71)

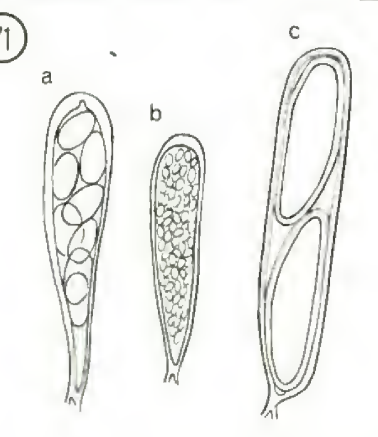

(73)
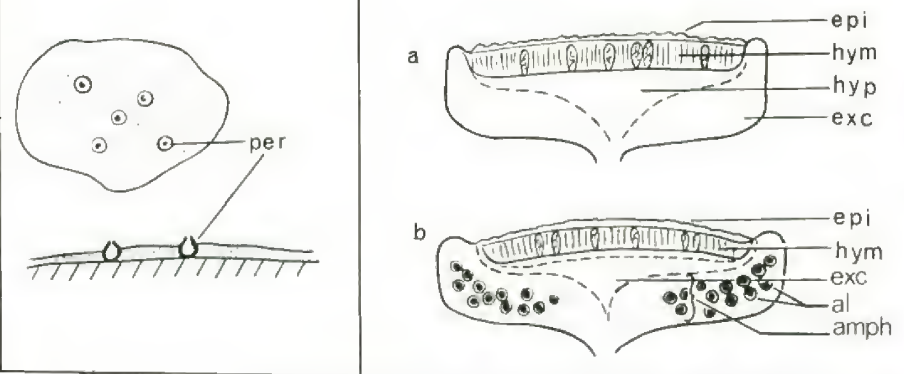

(72)

(67)
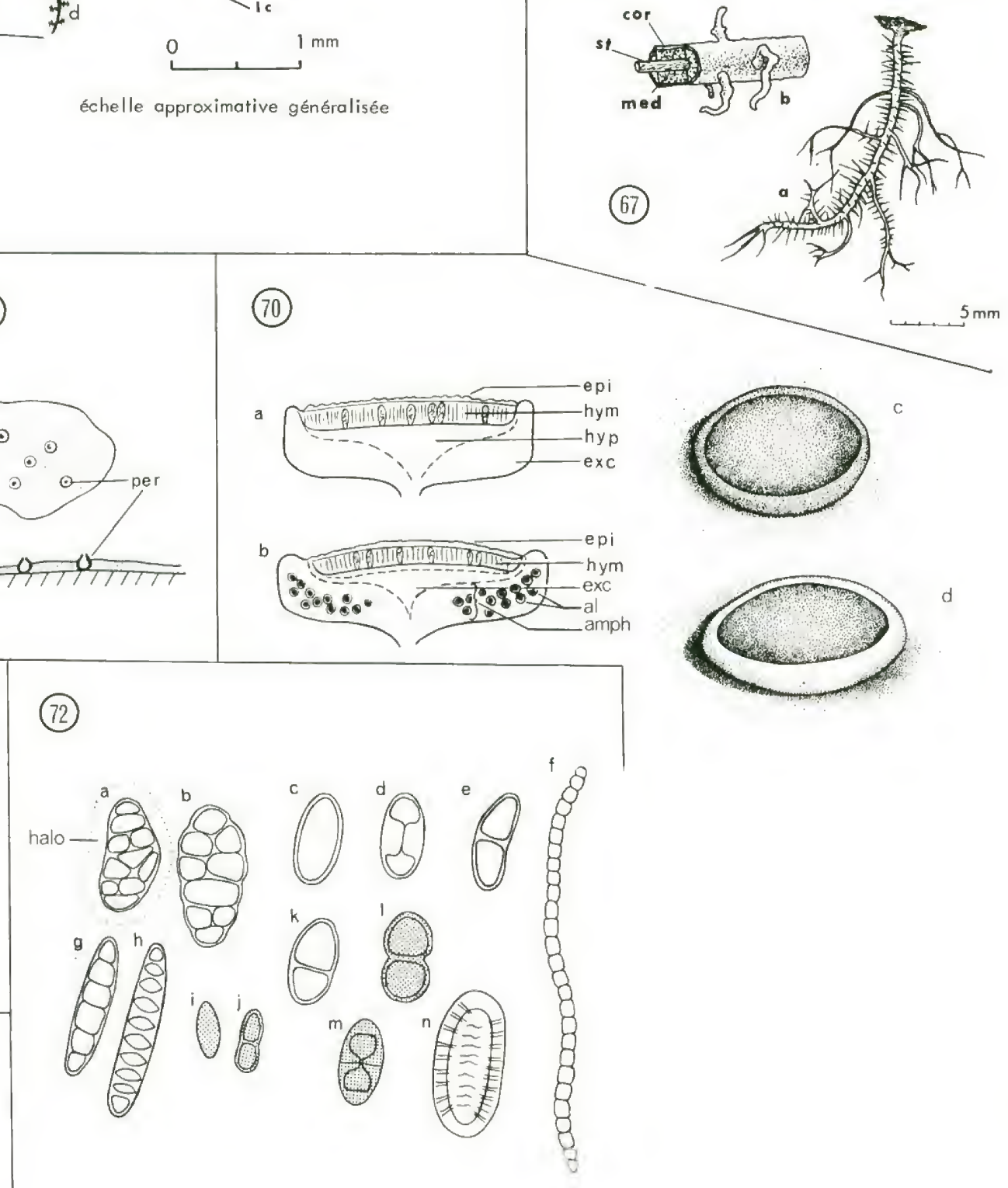

(74)

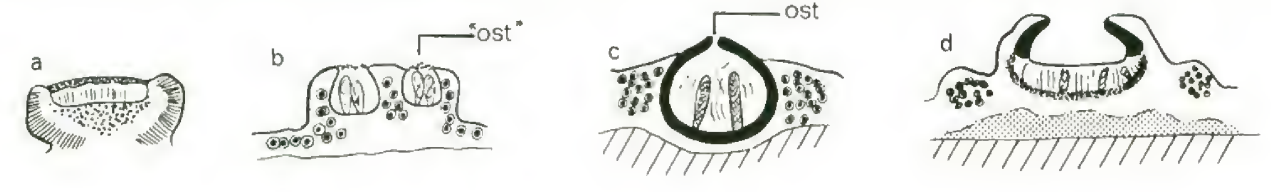




\section{LISTE ET INDEX DES LICHENS}

Dans la liste qui suit, je n'ai fait des renvois que dans le cas des synonymes très récents. Ces synonymes ont été écrits en italique. Quant aux taxons dont le nom apparaît entre crochets, leur présence n'est pas reconnue avec certitude dans la région d'ottawa, mais on doit s'attendre à les trouver. Les abréviations ON (Ontario) et QU (Québec) situées après chaque épithète servent à indiquer la situation des taxons par rapport à la rivière Outaouais. La référence de tous les endroits dans le texte où les taxons sont mentionnés est indiquée; le numéro des pages en italique renvoie aux illustrations.

\section{ACAROSPORA}

badiofusca (Nyl.) Th. Fr.

ON

Pages

canadensis Magn.

ON

21,37

fuscata (Schrad.) Arn.

ON QU

glaucocarpa (Wahlenb. ex Ach.) Koerb.

ON QU

37

37

18,37

37

\section{ANAPTYCHIA}

palmulata (Michx.) Vain.

QU

103

ANISOMERIDIUM

willeyana (R. Harris) R. Harris, ined. ON QU

39

\section{ARTHONIA}

$\begin{array}{lll} & & 20,29,37 \\ \text { ON } & \text { QU } & 29,37 \\ & \text { QU } & 38 \\ & \text { QU } & 38 \\ \text { ON } & \text { QU } & 38 \\ \text { ON } & \text { QU } & 24,38 \\ & \text { QU } & 38\end{array}$

\section{ARTHOPYRENIA}

25,38

punctiformis Mass.

ON

38

wizleyana R. Harris = Anisomeridium $w$.

\section{ARTHOTHELIUM}

anastomosans (Ach.) Arn.

ruanideum Nyl. ex Rehm in Rabenh.

spectabile (Flot. ex Fr.) Mass.

ASPICILIA

cinerea (L.) Koerb. var. cinerea

$\begin{array}{lll} & & 19,39 \\ & \text { QU } & 39 \\ \text { ON } & \text { QU } & 39 \\ & \text { QU } & 39 \\ & & \\ \text { ON } & \text { QU } & 21,39 \\ & 18,40\end{array}$




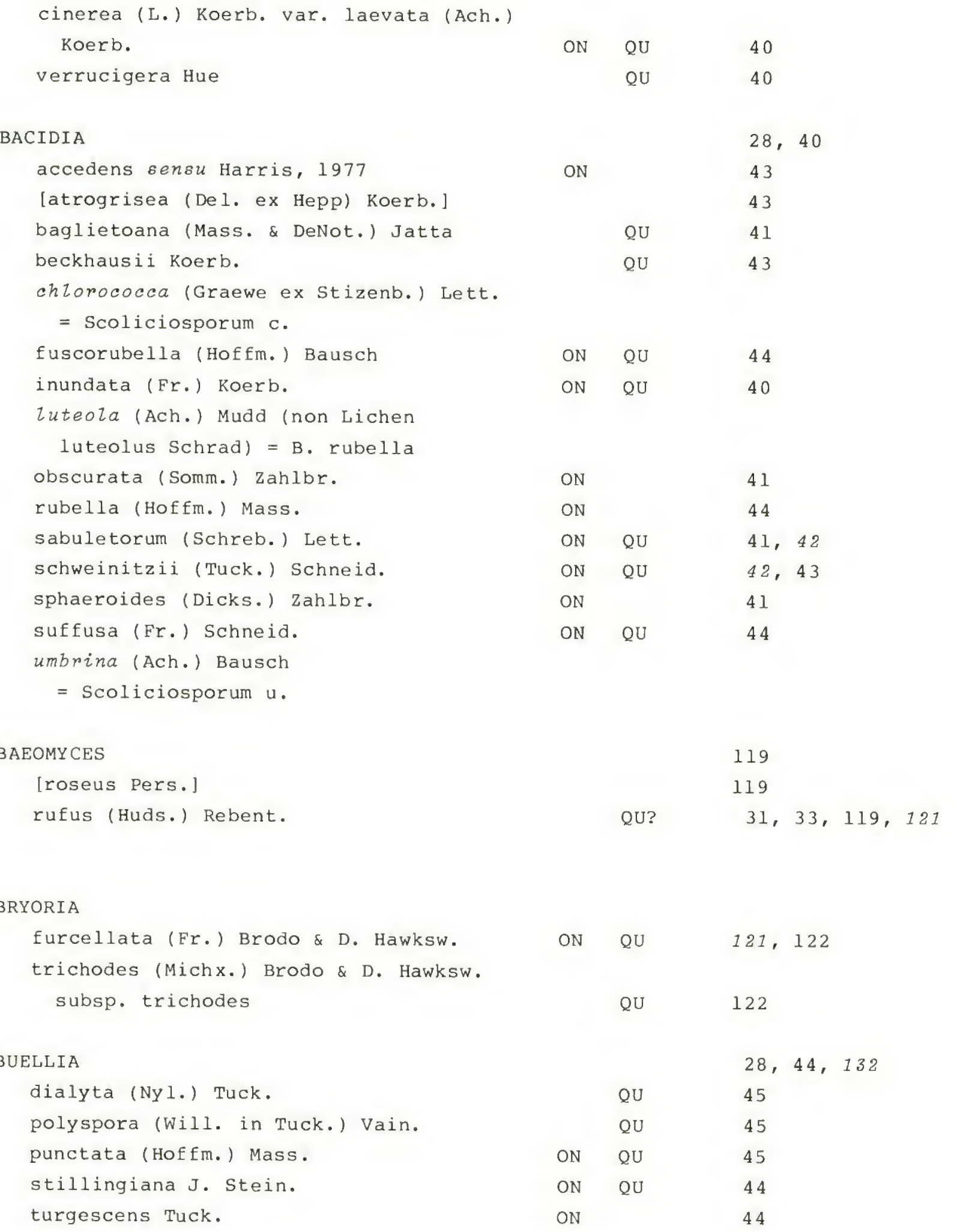


CALICIALES

\section{CALICIUM}

trabinellum (Ach.) Ach.

\section{CALOPLACA}

arenaria (Pers.) Muell. Arg.

ON

aurantiaca auct. non (Light.) Th. Fr.

$=$ C. Elavorubescens

cerina (Ehrh.) Th. Er.

citrina (Hoffm.) Th. Er.

feracissima Magn.

flavorubescens (Huds.) Laund.

(Syn. C. aurantiaca auct.)

flavovirescens (Wulf.) Dalla Torre \&

Sarnth.

holocarpa (Hoffm.) Wade

microphyllina (Tuck.) Hasse

ulmorum (Fink) Fink

sp. (parasite sur Physciopsis)

ON

ON

ON

ON

ON
16,45

132 ,

QU

24,46

$17,19,47,132$

47

48

QU

49

47

47,48

QU

48

ON QU

$\mathrm{ON} Q \mathrm{QU}$

QU

47

48,48

48

49

47

\section{CANDELARIA}

concolor (Dicks.) B. Stein var. concolor

ON QU

80

concolor (Dicks.) B. Stein var. effusa

(Tuck.) Burnh.

ON QU

34,49

fibrosa (Fr.) Muell. Arg.

ON QU

80

\section{CANDELARIELLA}

aurella (Hoffm.) Zahlbr.

efflorescens R. Harris \& Buck, ined.

vitellina (Ehrh.) Muell. Arg.

xanthostigma (Ach.) Lett.

\section{CATILLARIA}

laureri Hepp ex Th. Fr.

ON QU

29,132

micrococca (Koerb.) Th. Fr.

= Micarea m.

ON

QU

$\mathrm{ON} \quad \mathrm{QU}$

ON QU

$17,19,49$

49

$17,18,34,49$

49

$18,34,49$

62

CETRARIA

arenaria Karnef.

ON

101,102

ciliaris Ach.

ON QU

1.05

halei W. Culb. \& C. Culb.

ON QU

ON QU
104,105

81 
pinastri (Scop.) S. Gray

sepincola (Ehrh.) Ach.

\section{CETRELIA}

olivetorum (Nyl.) W. Culb \& C. Culb.

ON

QU

96

\section{CHAENOTHECA}

brunneola (Ach.) Muell. Arg.

QU

QU

47

stemonea (Ach.) Muell. Arg.

\section{CHAENOTHECOPSIS}

debilis (Turn. \& Borr. in Schaer.)

$$
\text { L. Tibell }
$$

ON

\section{CLADINA}

mitis (Sandst.) Hale \& W. Culb.

ON QU

ON QU

ON QU

110,111

rangiferina (L.) Nyl.

stellaris (Opiz) Brodo

\section{CLADONIA}

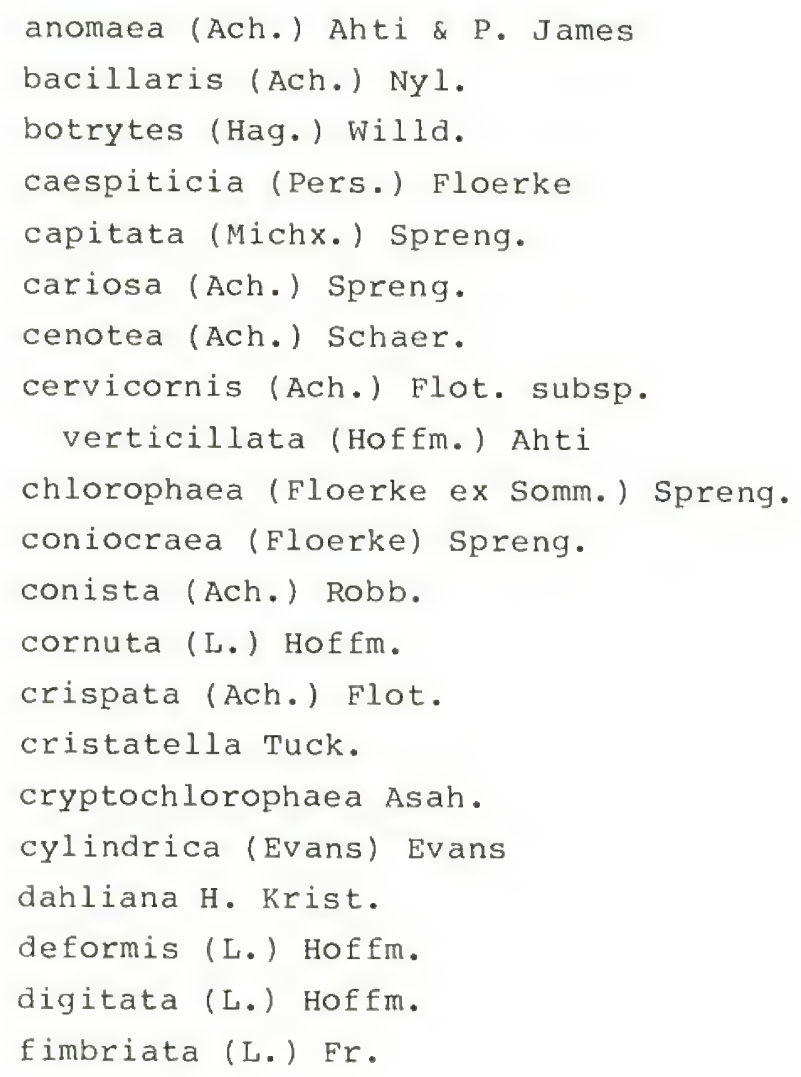

$\begin{array}{llcc}\text { ON } & & 119 \\ \text { ON } & \text { QU } & 112,117 \\ \text { ON } & & 116 & \\ \text { ON } & & 33,116 \\ \text { ON } & & 116 & \\ \text { ON } & \text { QU } & 32,117 \\ \text { ON } & \text { QU } & 112 & \\ & & & \\ \text { ON } & \text { QU } & 111,115 \\ \text { ON } & \text { QU } & 111,114 \\ \text { ON } & \text { QU } & 111,114 \\ \text { ON } & \text { QU } & 115 & \\ \text { ON } & & 118 & \\ \text { ON } & & 113 & \\ \text { ON } & \text { QU } & 110,132 \\ & \text { QU } & 114 & \\ \text { ON } & \text { QU } & 118 & \\ \text { ON } & & 32,116 \\ \text { ON } & \text { QU } & 110,112 \\ & \text { QU } & 110,112 \\ \text { ON } & \text { QU } & 114\end{array}$




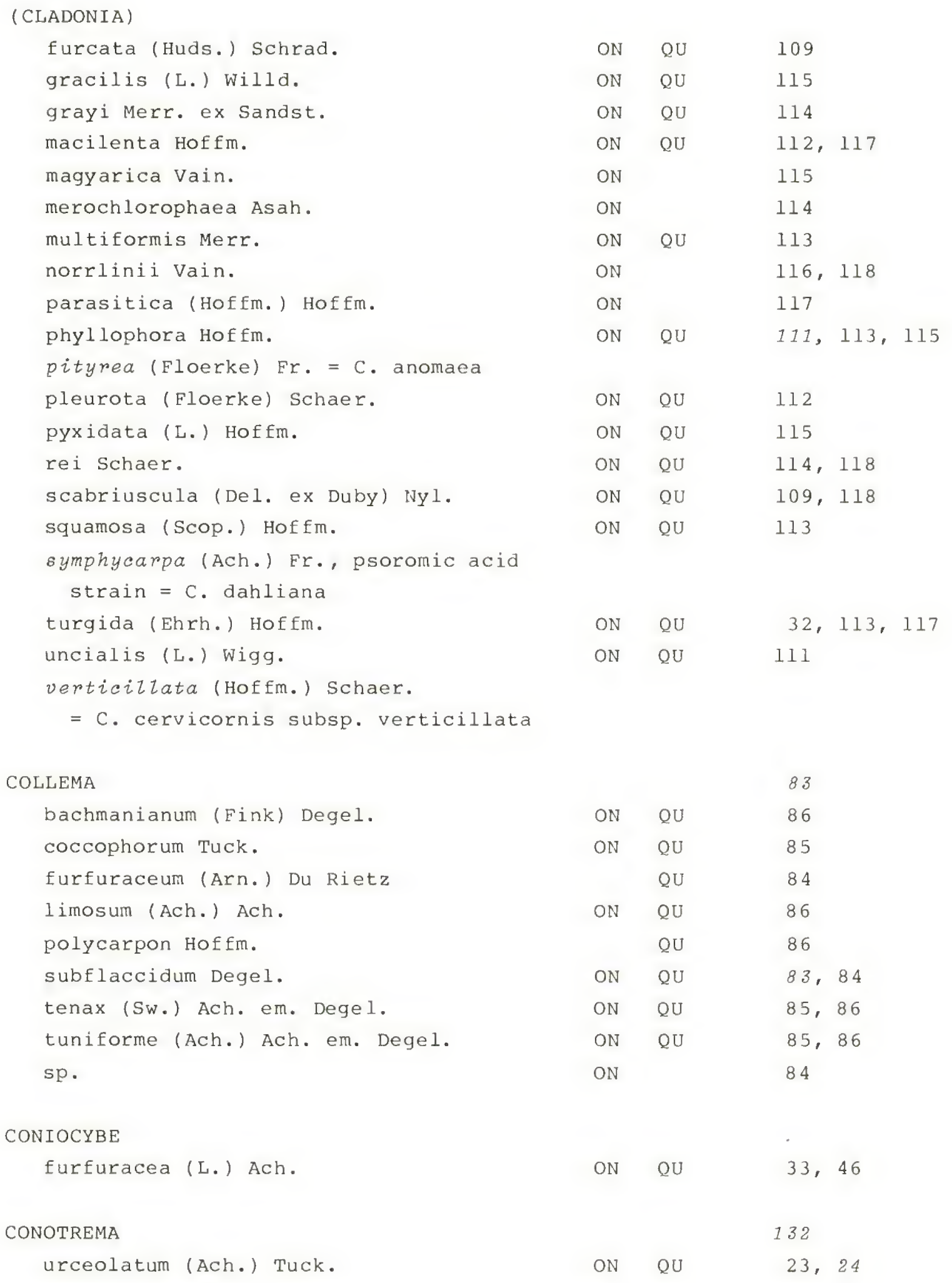




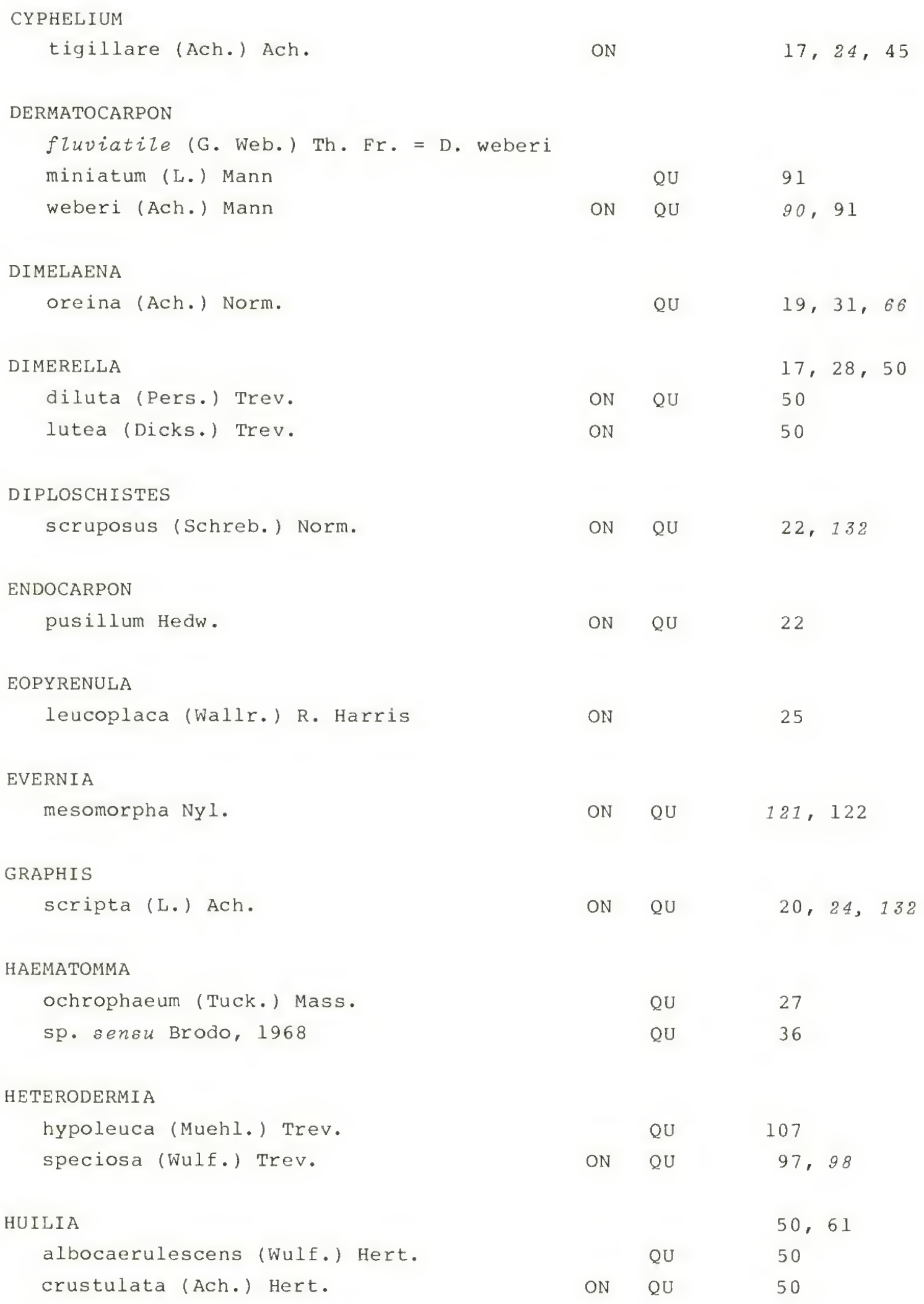


(HUILIA)

macrocarpa (DC.) Hert.

ON QU

50,63

HYMENELIA

lacustris (With.) Poelt \& Vezda

(Syn. Lecanora Lacustris)

QU

$19,39,56$

HYPOCENOMYCE

anthracophila (Nyl.) P. James \& Schneid.

QU

67

friesii (Ach.) P. James \& Schneid.

ON QU

33,67

scalaris (Ach.) Choisy

ON QU

67

$33,63,67$

HYPOGYMNIA

physodes (L.) Nyl.

ON QU

92,93

LASALLIA

papulosa (Ach.) Llano

QU

90,91

LECANIA

dimera (Nyl.) Th. Fr.

cyrtella (Ach.) Th. Fr.

ON? QU

27,50

50,132

$\mathrm{ON} \quad \mathrm{QU}$

51

\section{LECANORA}

allophana (Ach.) Nyl.

ON OU

$17,18,26,45$,

51,132

caesiorubella Ach. subsp. caesiorubella

ON QU

53

cenisia Ach.

ON QU

51

[chlarotera Nyl.]

chrysoleuca (Sm.) Ach.

cinereofusca Magn.

crenulata (Dicks.) Nyl.

dispersa (Pers.) Somm.

galactinula Vain.

55

ON QU 57

ON QU 55

QU 59

ON QU 59

[gangaleoides Nyl.]

glabrata (Ach.) Malme

QU

58

hagenii Ach.

ON QU

$53,54,66$

impudens Degel.

$\mathrm{ON} Q \mathrm{QU}$

51,56

imshaugii Brodo, ined.

ON QU

36,51

ON QU

53

Lacustris (With.) Nyl. = Hymenelia 1 .

muralis (Schreb.) Rabenh.

ON QU

$19,31,57$

opiniconensis Brodo, ined.

QU

57 


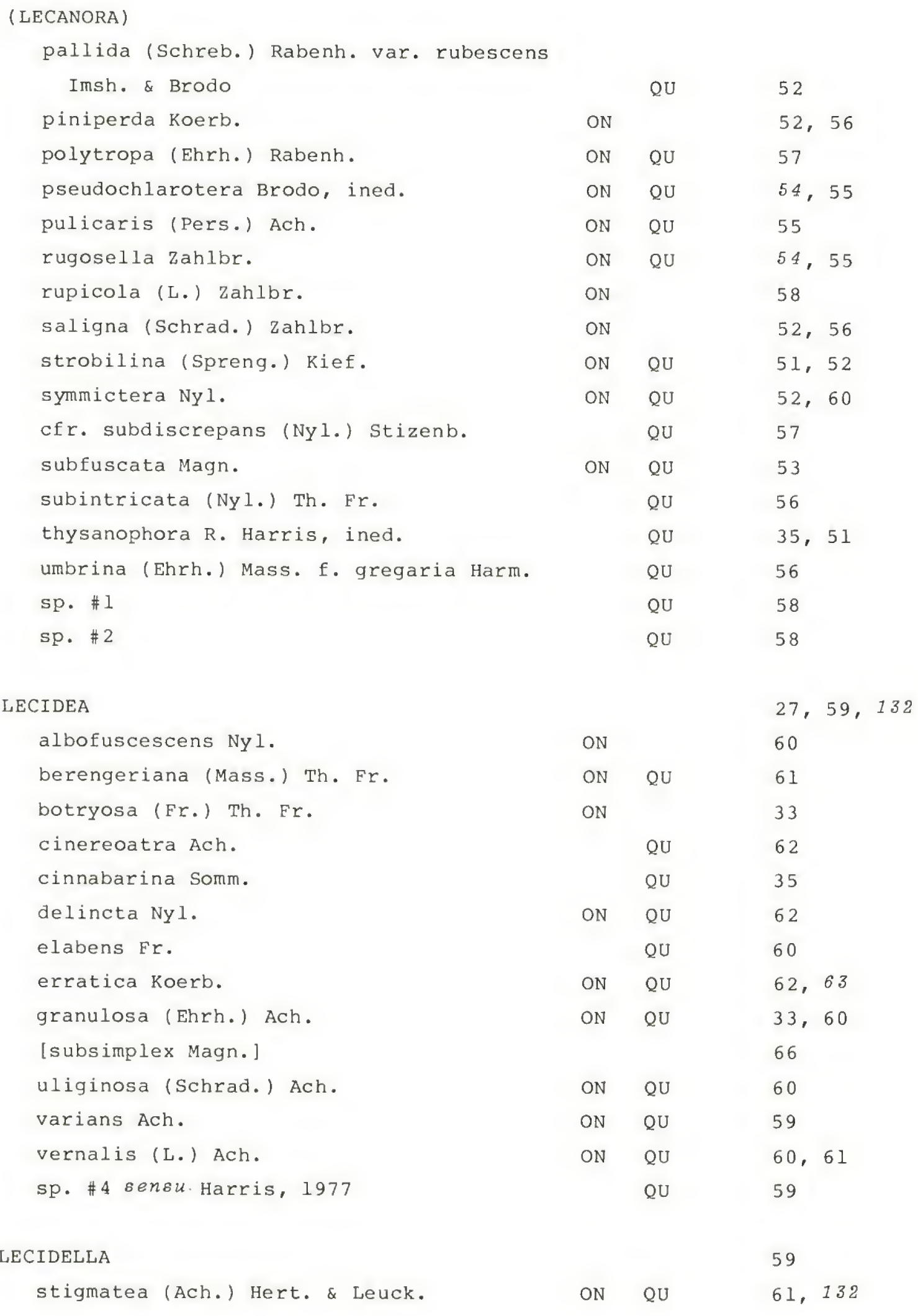


LEMP HOLEMMA

myriococcum (Ach.) Th. Fr.

ON QU

85

sp.

ON

85

\section{LEPRARIA}

finkii (B. de Lesd.) R. Harris, ined.

ON

QU

31,35

incana (L.) Ach.

ON

$\mathrm{QU}$

31,35

lobificans Nyl. s.l.

QU $\quad 30$

membranacea (Dicks.) Vain.

$\mathrm{QU}$

30

zonata Brodo

$\mathrm{QU}$

31

sp \#2 sensu Harris, 1977

QU

30

35

LEPTOGIUM

83

azureum (Sw.) Mont.

ON

82

burnetiae Dodge var. hirsutum (Sierk)

P.M. Joerg.

ON

cyanescens (Ach.) Koerb.

ON

QU

82

dactylinum Tuck. in Nyl.

ON

QU

82,83

juniperinum Tuck.

ON

82

lichenoides (L.) Zahlbr.

ON

82

QU

82,83

LEPTORHAPHIS

epidermidis (Ach.) Th. Er.

LOBARI A

pulmonaria (L.) Hoffm.

ON QU

$77,93,94$

quercizans Michx.

ON QU

106

\section{[ LOPADIUM]}

[pezizoideum (Ach.) Koerb.]

MENEGAZZIA

terebrata (Hoffm.) Mass.

ON

QU

92

MICAREA

bauschiana (Koerb.) Wirth \& Vezda

QU

29,62

melaena (Nyl.) Hedl.

QU

62

micrococca (Koerb.) Brodo, ined. (Syn.

Catizlaria micrococeal

QU

41,64

viridescens (Schrad.) Brodo

QU

62

$27,33,62$ 
parietinum (Ach. ex Schaer.) D. Hawksw.

(Syn. M. subtize (Pers.) Szat.)

MYCOPORUM

pycnocarpum Nyl.

QU

22

NEPHROMA

bellum (Spreng.) Tuck.

ON QU

88

parile (Ach.) Ach.

ON QU

87

resupinatum (L.) Ach.

ON

88

\section{OCHROLECHIA}

androgyna (Hoffm.) Arn. s.s.

arborea (Ljubitz.) Almb.

pseudopallescens Brodo, ined.

rosella (Tuck.) Vers.

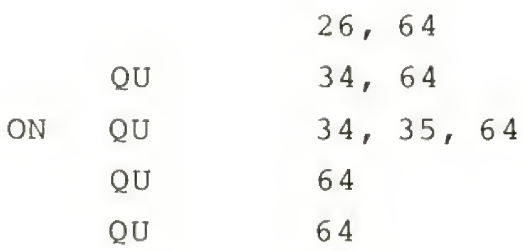

OPEGRAPHA

pulicaris (Hoffm.) Schrad.

PANNARIA

ON

86

ahlneri P.M. Joerg.

ON

87

leucophaea (Vahl) P.M. Joerg.

ON QU

25,88

\section{PARMELIA S.I.}

aurulenta Tuck.

[baltimorensis Gyeln. \& For.]

132
ON QU $\quad 100$

bolliana Muell. Arg.

81

borreri (Sm.) Turn.

ON QU

107

caperata (L.) Ach.

ON QU

96,100

conspersa (Ehrh. ex Ach.) Ach.

81

crinita Ach.

QU

80

cumberlandia (Gyeln.) Hale

ON QU

95

disjuncta Erichs.

ON QU

79,80

exasperatula Nyl.

$Q U$

98,100

flaventior stirt.

QU

94

ON

81

galbina Ach.

ON QU

106

olivacea (L.) Ach.

QU

105

plittii Gyeln.

QU

80

rudecta Ach.

ON QU

94 


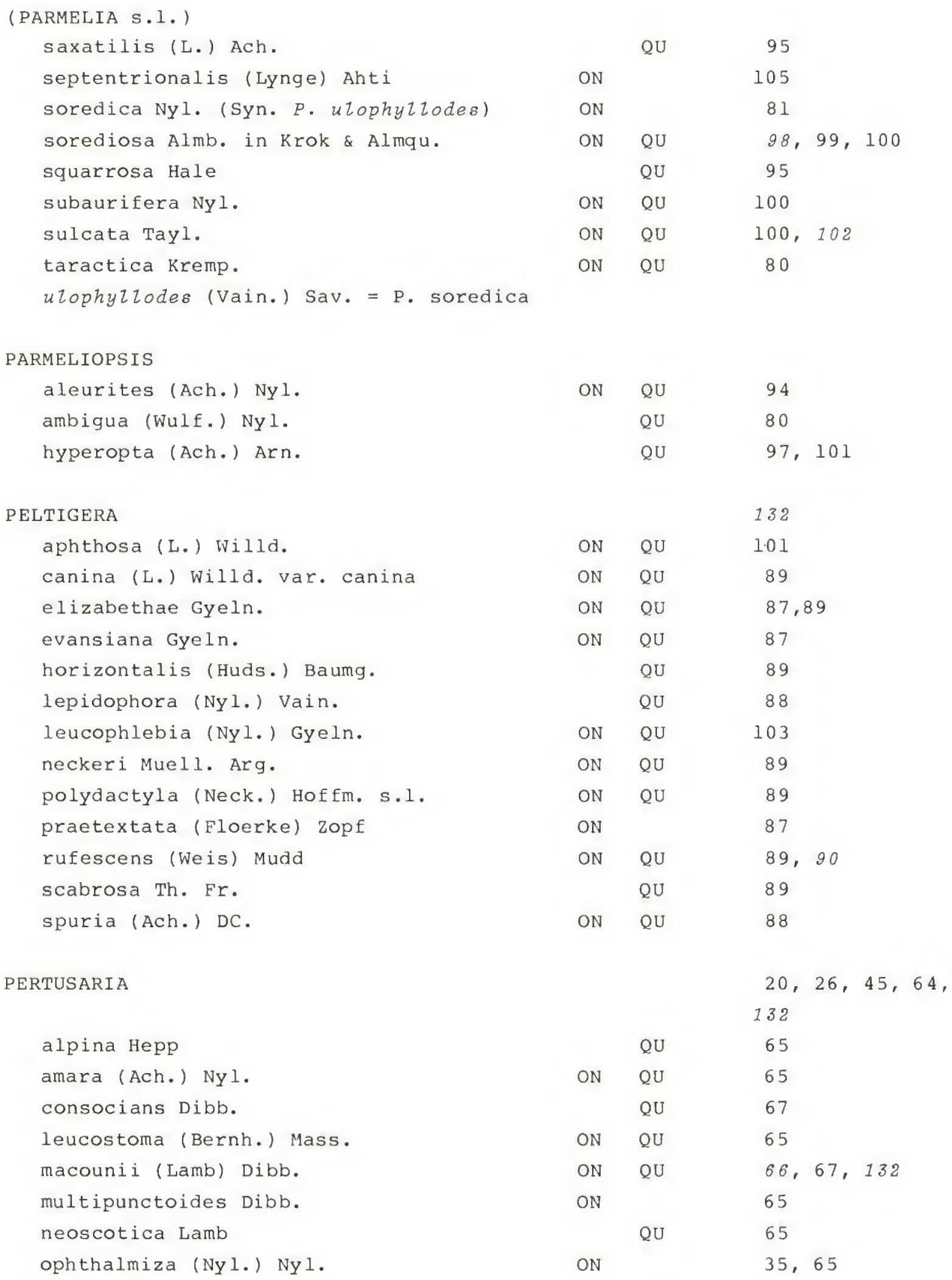


(PERTUSARIA)

rubefacta Erichs.

trachythallina Erichs.

velata (Turn.) Nyl.

waghornei Hult.

$\begin{array}{lll} & \text { QU } & 65 \\ & \text { QU } & 36,65 \\ \text { ON } & \text { QU } & 65 \\ & \text { QU } & 65\end{array}$

\section{PHAEOCALICIUM}

curtisii (Tuck.) L. Tibell

\section{PHAEOPHYSCIA}

\begin{tabular}{|c|c|c|c|}
\hline adiastola (Essl.) Essl. & ON & QU & 99 \\
\hline cernohorskyi (Nadv.) Essl. & ON & QU & 99 \\
\hline ciliata (Hoffm.) Moberg & ON & QU & 106 \\
\hline decolor (Kashiw.) Essl. & & QU & 106 \\
\hline hirtella Essl. & ON & QU & 106 \\
\hline hispidula (Ach.) Essl. & ON & QU & 97,99 \\
\hline $\begin{array}{l}\text { imbricata (Vain.) Essl. (Syn. Physcia } \\
\text { Zacinulata N. Amer. auct. non Muell. } \\
\text { Arg.) }\end{array}$ & & QU & 103 \\
\hline pusilloides (Zahlbr.) Essl. & ON & & 99 \\
\hline rubropulchra (Degel.) Essl. & ON & QU & 95,98 \\
\hline sciastra (Ach.) Moberg & ON & QU & 99 \\
\hline
\end{tabular}

\section{PHLYCTIS}

argena (Ach.) Flot.

\section{PHYSCIA}

ON

QU

132

adscendens (Th. Fr.) Oliv.

aipolia (Ehrh.) Hampe

ON

caesia (Hoffm.) Lett.

ON

QU

104,107

chloantha Ach.

ON QU

101

dubia (Hoffm.) Lett.

ON

millegrana Degel.

phaea (Tuck.) Thoms.

QU

96,97

stellaris (L.) Nyl.

QU

97,101

97,98

tribacoides Nyl.

ON

107

107

ON

101

PHYSCIOPSIS

adglutinata (Floerke) Choisy

ON

QU

99

PHYSCONIA

detersa (Nyl.) poelt

ON

QU 


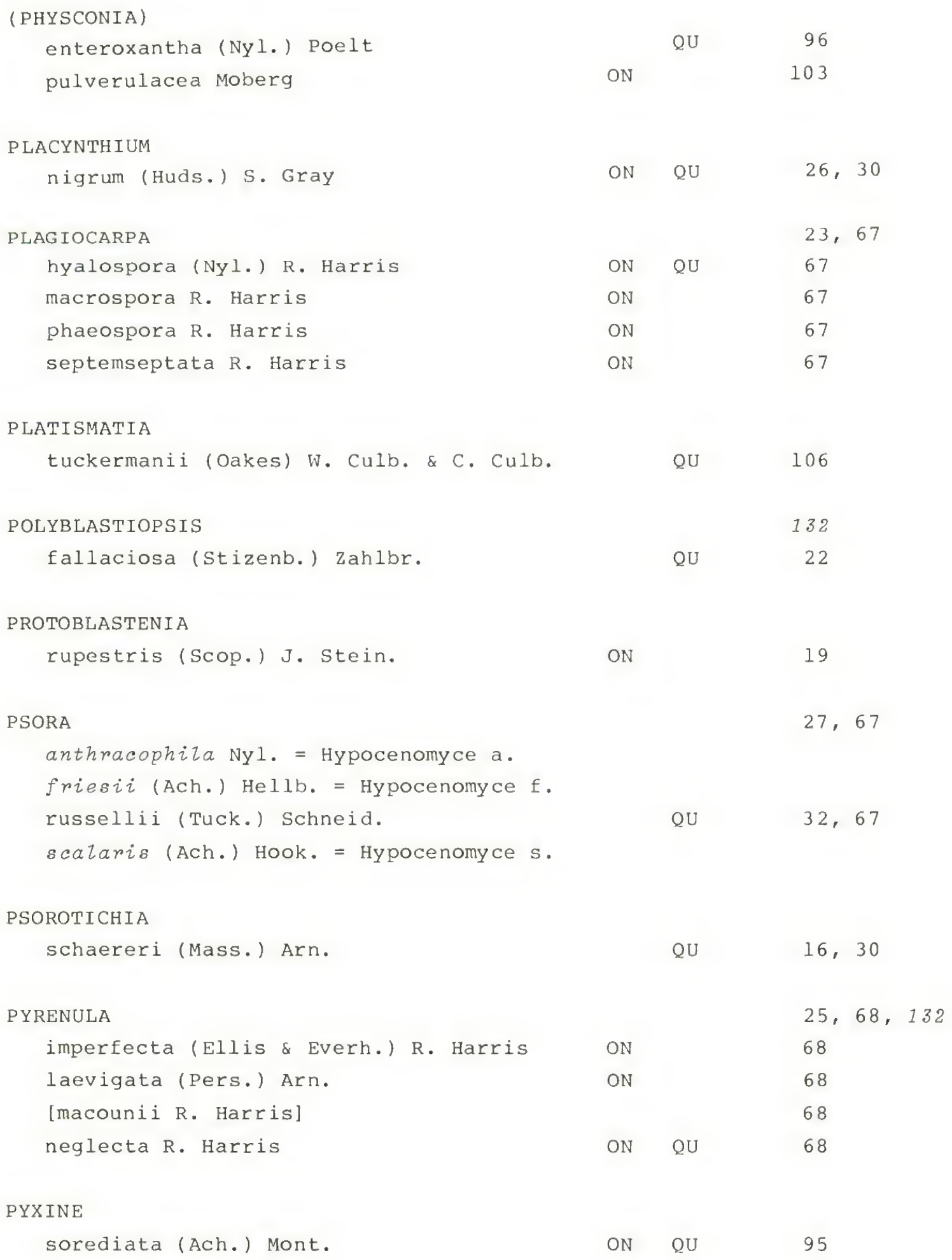


RAMALINA

americana Hale

dilacerata (Hoffm.) Hoffm.

ON

123

intermedia Nyl.

obtusata (Arn.) Bitt.

$\begin{array}{lll} & \text { QU } & 123 \\ & \text { QU } & 121,122 \\ \text { ON } & & 122\end{array}$

\section{RHI ZOCARPON}

eupetraeum (Nyl.) Arn.

grande (Floerke ex Flot.) Arn.

hochstetteri (Koerb.) Vain.

lavatum (Ach. ex Fr.) Hazl.

[lecanorinum And.]

122

obscuratum (Ach.) Mass.

tetramerum (Vain.) Vain.

$$
19,28,68
$$

QU

69

ON

QU

69

QU

29,69

QU

69,132

68

QU

70

ON

69

RINODINA

ascociscana (Tuck.) Tuck.

ON QU

$26,70,132$

bischoffii (Hepp) Mass.

dakotensis Magn.

halei Magn.

novoconfragosa sheard, ined.

palustris Will. ex Sheard

polyspora Th. Fr.

[populicola Magn.]

subminuta Magn.

verrucosa Merr. ex Sheard, ined.

ON

$Q \mathrm{U}$

70

70,71

71,71

71,71

QU

70

ON QU

72

ON

72

72

QU

71

ON

70

\section{SARCOGYNE}

clavus (Ram.) Kremp.

$27,72,132$

regularis koerb.

privigna (Ach.) Mass.

simplex (Dav.) Nyl.

SCOLICIOSPORUM

chlorococcum (Graewe ex Stenh.) Vezda

$\begin{array}{ll}\text { ON } & \text { QU } \\ \text { ON } & \text { QU }\end{array}$

41,42

umbrinum (Ach.) Arn.

40

SOLORINA

saccata (L.) Ach.

ON QU

$76,90,92$ 


\section{SPHINCTRINA}

microcephala Koerb.

STAUROTHELE

22,73

catalepta (Ach.) Blomb. \& Forss.

ON

73

diffractella (Nyl.) Tuck.

fissa (Tayl.) Zw.

STEREOCAULON

dactylophyllum Fr.

saxatile Magn.

tomentosum Fr.

THELIDIUM

decipiens (Hepp) Kremp.

QU

23

THROMBIUM

epigaeum (Pers.) Wallr.

$\mathrm{QU}$

22

THYREA

pulvinata (Schaer.) Mass.

TRAPELIA

involuta (Tayl. in Mack.) Hert.

ON

QU

84

TRYPETHELIUM

virens Tuck. in Darl.

ON QU

23

UMB I L I CAR I A

deusta (L.) Baumg.

mammulata (Ach.) Tuck.

muehlenbergii (Ach.) Tuck.

vellea (L.) Ach.

USNEA

filipendula stirt.

hirta (L.) Wigg.

subfloridana stirt.

$\begin{array}{rrr} & 132 \\ & & 91 \\ & \text { QU } & 92 \\ & \text { QU } & 92 \\ & \text { QU } & 92\end{array}$

$\begin{array}{llll} & \text { QU } & 120 \\ \text { ON } & \text { QU } & 120,121 \\ \text { ON } & \text { QU } & 120,132\end{array}$




\section{VERRUCARIA}

aethiobola Wahlenb. ex Ach.

calciseda DC.

calkinsiana Serv.

fuscella (Turn.) Ach.

muralis Ach.

nigrescens pers.

nigrescentoidea Fink

virens Nyl.

\section{XANTHORIA}

elegans (Link) Th. Fr.

fallax (Hepp) Arn.

polycarpa (Ehrh.) oliv.
22,73

74

66,73

75

74

74

74

74

74

$\begin{array}{ll}\text { ON } & \text { QU }\end{array}$

$\begin{array}{lll} & \text { QU } & 78 \\ \text { ON } & \text { QU } & 78,79 \\ \text { ON } & & 78\end{array}$






\section{RECENT SYLLOGEUS TITLES/TITRES RECENTS DANS LA COLLECTION SYLLOGEUS}

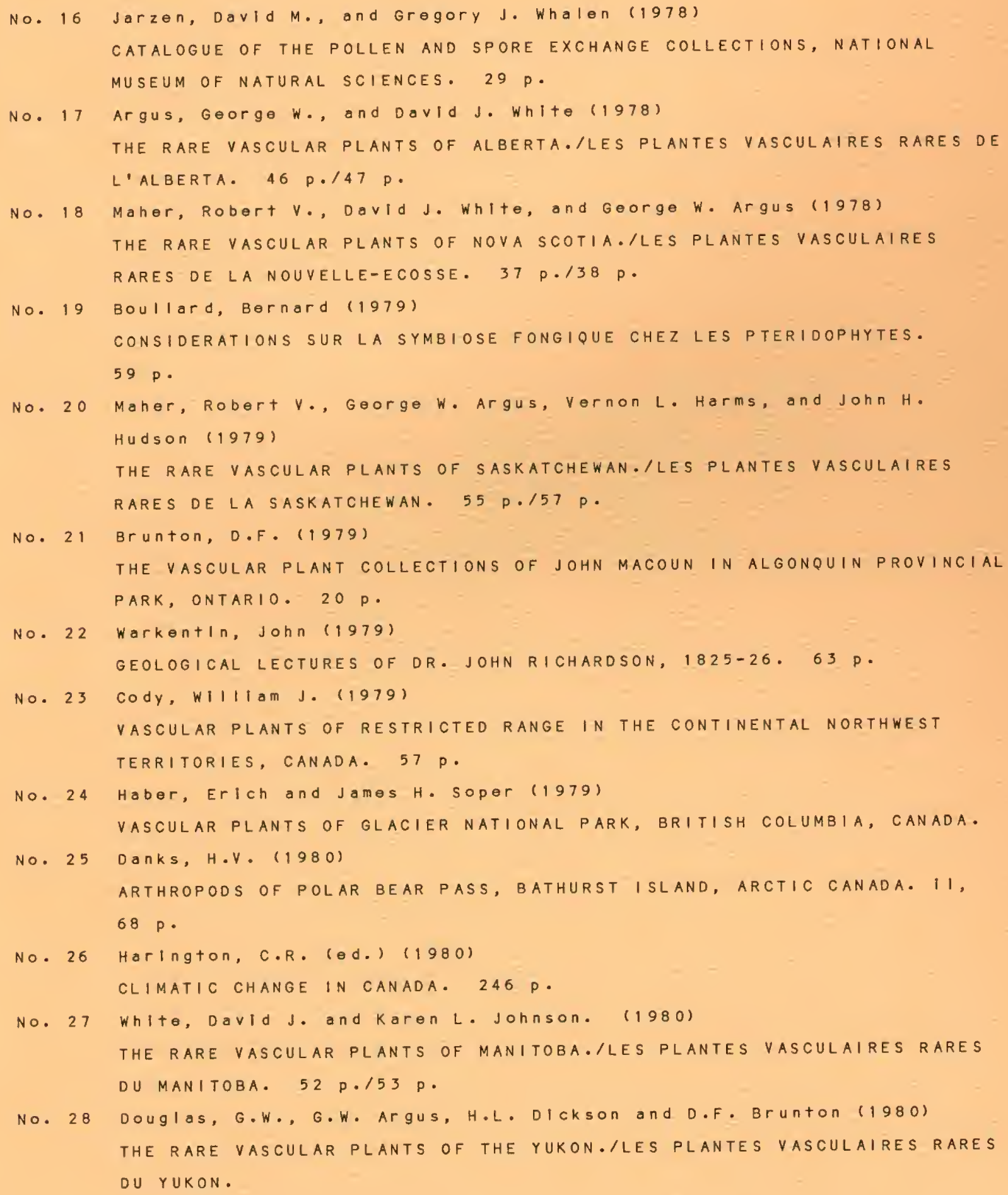




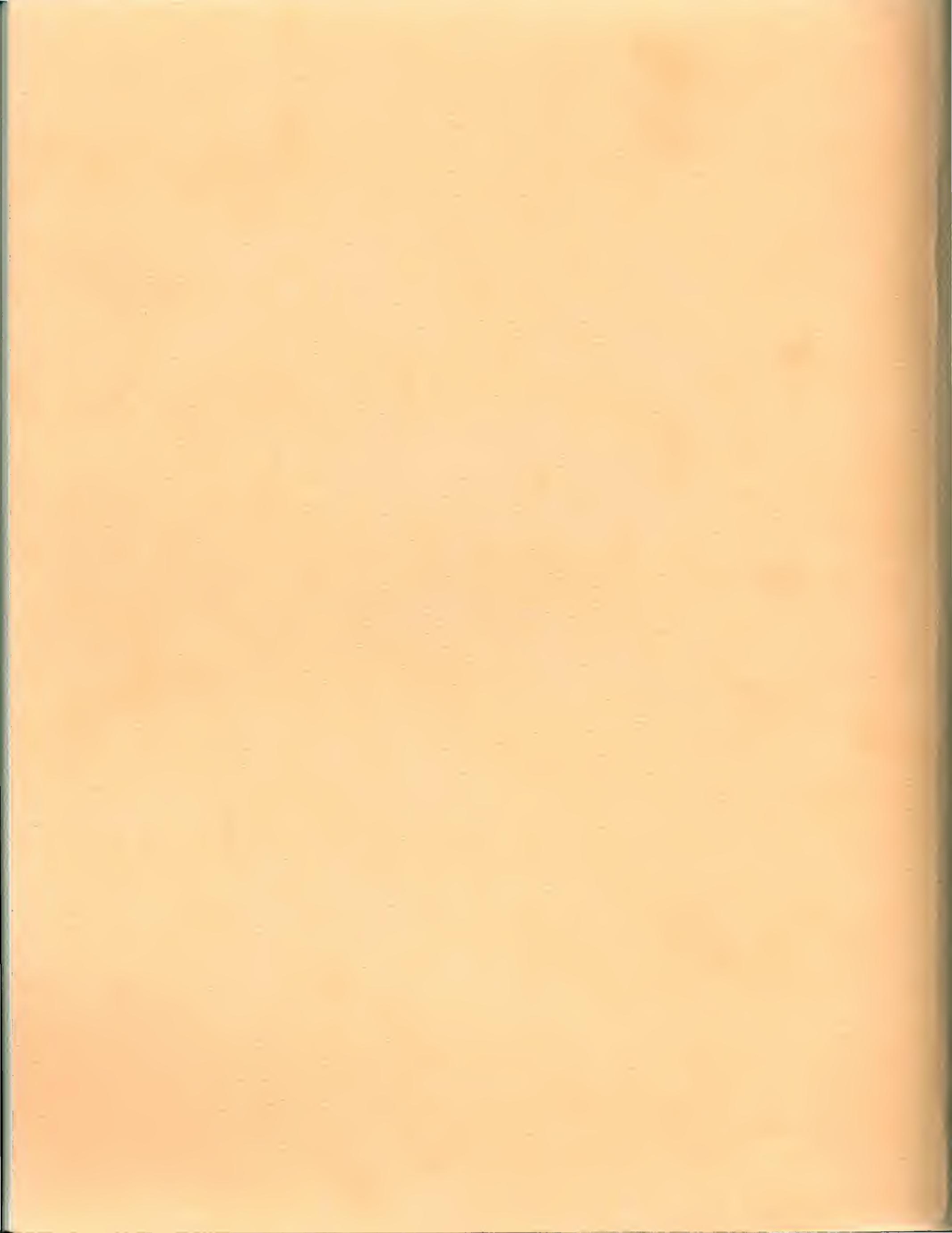

